\title{
ASSOCIATIONS BETWEEN ABDOMINAL ADIPOSITY, EXERCISE, MORBIDITY AND MORTALITY
}

\author{
by
}

Jennifer L. Kuk

A thesis submitted to the School of Kinesiology and Health Studies

in conformity with the requirements for the

Degree of Doctor of Philosophy

\author{
Queen's University \\ Kingston, Ontario, Canada
}

May, 2007

Copyright (C) Jennifer L. Kuk, 2007 


\section{Abstract}

The increasing prevalence of abdominal obesity worldwide poses a serious public health problem and hence, presents a target for research designed to improve the assessment or treatment of abdominal obesity. Specifically, the first study in this thesis investigated the influence of age and gender on visceral (VAT) and abdominal subcutaneous adipose tissue (ASAT) for a given waist circumference (WC) in 481 men and women varying widely in age and BMI. Significant gender differences in VAT and ASAT for a given WC were observed, however, only the relationship between WC and VAT was substantially influenced by age.

The second study examined whether the associations between VAT, ASAT and the metabolic syndrome (MetS) were altered depending on measurement methodology used to assess VAT and ASAT. The odds ratio (OR) for MetS was higher for total VAT volume $(\mathrm{OR}=7.26)$ and the partial volumes at T12-L1 $(\mathrm{OR}=7.46)$ and $\mathrm{L} 1-\mathrm{L} 2(\mathrm{OR}=8.77)$ compared to the classic L4-L5 $(\mathrm{OR}=3.94)$ measurement. The OR for MetS was not substantially different among the ASAT measures (OR 2.6). Measurement site for VAT, but not ASAT, has a substantial influence on the magnitude of the association with MetS.

The third study examined the independent associations between VAT, ASAT, liver fat and all-cause mortality in 291 men (97 decedents and 194 controls, mortality follow-up of $2.2 \pm 1.3$ years). In a model including VAT, ASAT, liver fat, age, and length of follow-up, only VAT (1.93 [1.15-3.23]) remained a significant predictor of mortality. We concluded that VAT is a strong, independent predictor of all-cause mortality in men.

The purpose of the final study was to determine the effect of aerobic exercise dose (energy expenditure) on WC in sedentary, overweight/obese postmenopausal women 
$(n=424)$. The women were randomly assigned to a control group or one of three aerobic exercise groups that exercised at energy expenditures of 4-, 8-, or 12-kcal/kg body weight/week. By comparison to control, there were significant reductions in WC in the exercise groups $(\sim 3 \mathrm{~cm}, \mathrm{P}<0.05)$, which were independent of weight loss. However, the amount of exercise performed was not associated with reductions in WC in a dose dependent manner.

\section{Keywords:}

Visceral adipose tissue, waist circumference, cardiorespiratory fitness, liver fat, metabolic syndrome, age, gender 


\section{Co-Authorship}

The co-authors of the manuscripts contained within this thesis are Drs. Robert Ross (Chapter 3-6), SoJung Lee (Chapter 3), Steven B. Heymsfield (Chapter 3), Steven N. Blair (Chapter 4-6), Timothy S. Church (Chapter 4-6), Peter T. Katzmarzyk (Chapter 5) and Milton Z. Nichaman (Chapter 5). 


\section{Acknowledgements}

This is the second time in my life now that I have had to write an acknowledgement. The first time, at the end of my Master's, it seemed trivial since I knew the journey was not complete, and that nothing was going to change other than the letters at the end of my name. This time is different. After six years I will be leaving at the conclusion of this chapter, and with much more then the extra letters behind my name. I do not know what the future holds, but in looking back I can only say thanks to the people that have made this thesis possible.

Firstly, I would like to thank my parents who have supported me throughout my life. You have always challenged me to work hard and strive for lofty goals without fear of failure, and have loved me without condition. I thank God for guiding me and providing the people and opportunities I needed to succeed.

Next, I would like to thank Fred and my friends that have stuck by me over the last six years through the good times and bad. Despite the long periods of silence where I hibernated in my lab, you were always there when I needed you, to give me a hug, listen and even tolerated sporadic bouts of insanity and tears. Some of you I knew before Queen's, and some only since Queen's, but I hope that I will continue to know you all after Queen's. You know who you are and I am thankful everyday for each and every one of you.

I would also like to acknowledge my lab mates, and in particular SoJung. What can I say except that you've helped make the days go by a little quicker. I can't believe it's been six years, and despite our fights about who was going to monitor the grumpy subject, about who was going to wake up at 5am to do testing, or get Bob his coffee, 
we've managed not to kill each other (although there have been some close calls), and even become good friends. Thanks for the good times, and now after six years of watching everyone around me leave, it's finally my turn!!

Over the last six years, I have also had the privilege to work with some of the leading scientists in the field. To Dr. Steven Blair and Dr. Timothy Church I want to say thank you for giving me the opportunity to work with you for the last six years. To Elisa Priest, much of my success today is because of you and I can only say thank you. Without you, I think I would still be pulling files from Blue Mountain or some other remote location in Dallas for my Master's thesis, and there would be no way that my doctoral thesis would have ever come to fruition.

Finally, I would like to thank Dr. Ross for opening the door and having me as your student. Who would have guessed that a meeting in a hotel bar, a Blue Lite and a cracked glass would lead to this? For as long as I have known you, you have demanded excellence and challenged me to do better. Over the years, we have had several heated scientific discussions and many periods of frustration that have also been interrupted with the occasional successes. In looking back, I come to realize that you have always had the best of intentions, and I now realize that the process was probably often times more painful for you than me. My current and futures successes are indebted to you, and I only hope that I can one day meet the potential that you see in me and to make you proud. 


\section{Table of Contents}

Abstract

Co-Authorship

Acknowledgements

Table of Contents

List of Tables

List of Figures

List of Abbreviations

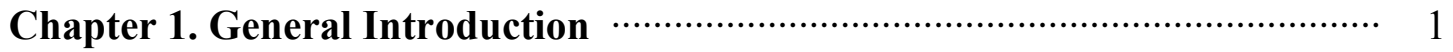

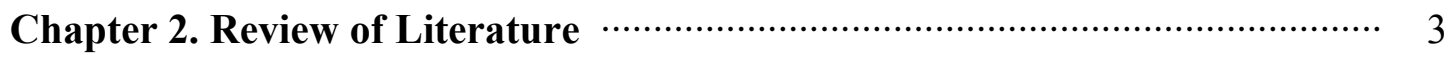

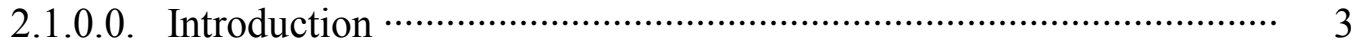

2.2.0.0. Abdominal Obesity and Health Risk …….................................... 3

2.3.0.0. Abdominal Adiposity and Health Risk …….................................. 5

2.3.1.0. Measurement of Abdominal Adiposity ….............................. 7

2.4.0.0. Liver Fat and Health Risk ………...................................... 9

2.4.1.0. Measurement of Liver Fat …........................................... 12

2.5.0.0. Skeletal Muscle Lipid and Health Risk …................................. 16

2.5.1.0. Measurement of Skeletal Muscle Lipid …............................ 16

2.6.0.0. Influence of Physical Activity and Exercise on Obesity and Health Risk ….................................................................................. 18

2.6.1.0. Influence of Physical Activity and Exercise on Obesity …….. 18

2.6.2.0. Influence of Physical Activity and Exercise on Health Risk $\cdots 20$

2.5.3.0. Dose Response Relationship between Exercise and Obesity Reduction …......................................................................... 22 


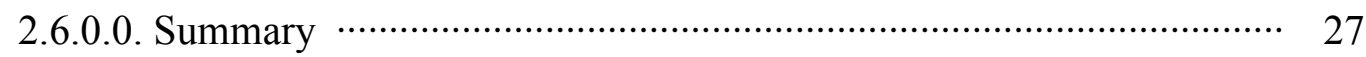

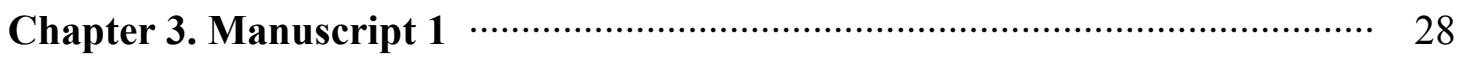

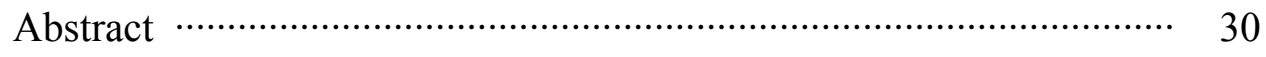

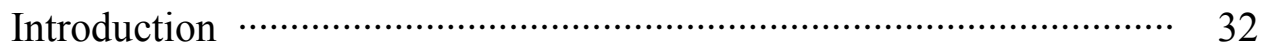

Research Design and Methods ……………............................ 34

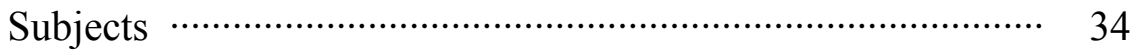

Anthropometric Measurements ………………………... 34

Measurement by Magnetic Resonance Imaging ……............. 35

Statistical Analyses …………………….................... 36

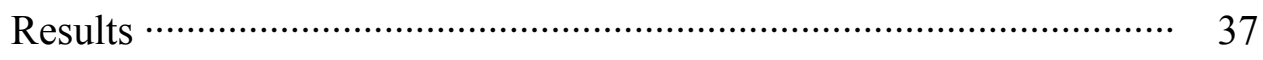

Discussion

Acknowledgements …….............................................. 43

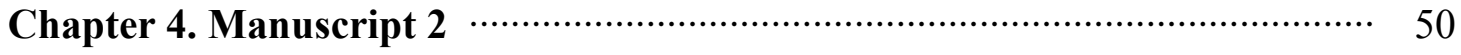

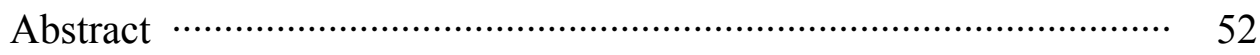

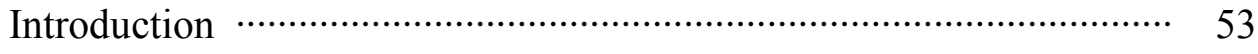

Research Design and Methods ……............................................ 55

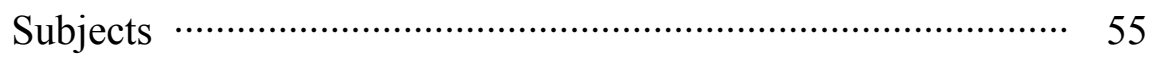

Biochemistry Analyses ……………………................ 55

Measurement of Abdominal AT by Computed Tomography … 56

Determination and Calculation of Abdominal AT Partial and

Total Volumes …............................................... 57

Statistical Analyses ……………………….............. 57

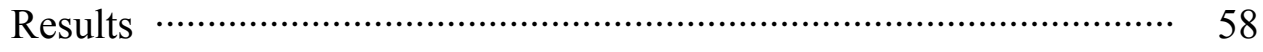




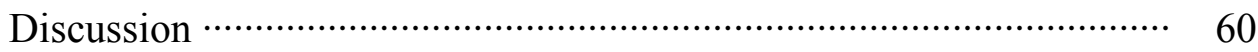

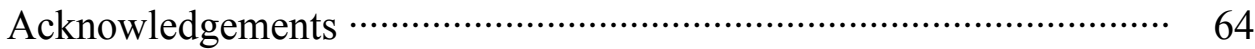

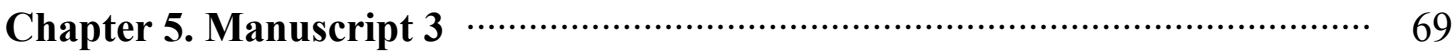

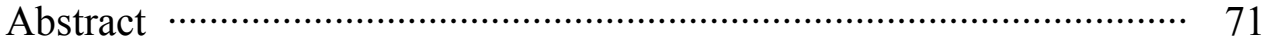

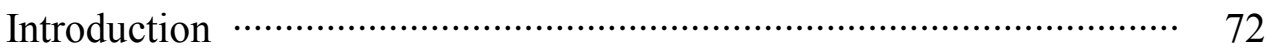

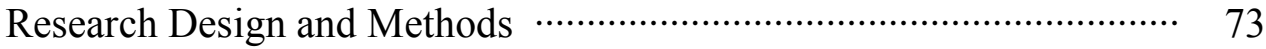

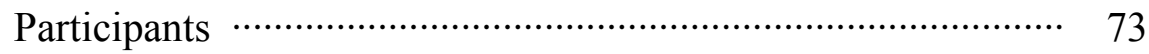

Procedures

Statistical Analyses …………………….................. 75

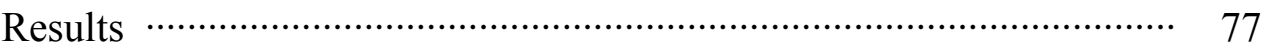

Discussion

Acknowledgements …….............................................. 82

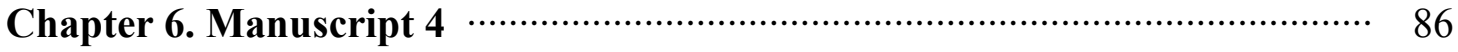

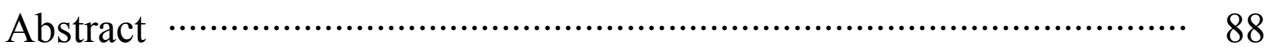

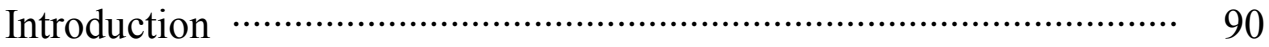

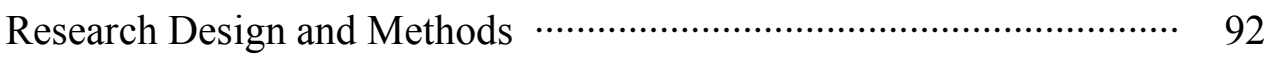

Subject Criteria

Clinical Examination ……………………................... 92

Computed Tomography Assessment …................................. 93

Maximal Oxygen Uptake Measurements ……....................... 95

Exercise Intervention ……......................................... 95

Statistical Analyses …................................................... 96 
Changes in Waist Circumference, Waist-to-Hip Ratio and Body Weight

Changes in the Computed Tomography Measures

Discussion

Acknowledgements

Chapter 7. General Discussion

References

\section{Appendices}

Appendix A Determination of Sample Size and Statistical Power …..............

Appendix B Anthropometric Data Collection Form - Queen's University …

Appendix C Electron Beam Computerized Tomography Scan Consent Form in Accordance with the Ethical Guidelines of The Cooper Institute Institutional Review Board

Appendix D Aerobics Center Longitudinal Study Self-Report Questionnaire - The Cooper Institute

Appendix E DREW Consent Form - The Cooper Institute

Appendix F DREW Electron Beam Tomography Disclosure and Consent Form - The Cooper Institute

Appendix G Letter to DREW Participants Regarding Randomization - The Cooper Institute 
Appendix H DREW Data Acquisition Timeline Form - The Cooper

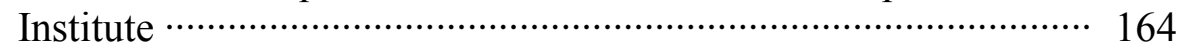

Appendix I DREW Anthropometric Data Form - The Cooper Institute …… 166

Appendix J DREW Maximal Oxygen Uptake Test Form - The Cooper Institute …………………………………………………… 168

Appendix K DREW Intervention Exercise Log - The Cooper Institute ……... 170

Appendix L Examples of Statistical Analyses Used in Manuscript 1 to $4 \cdots \cdots \cdot 174$

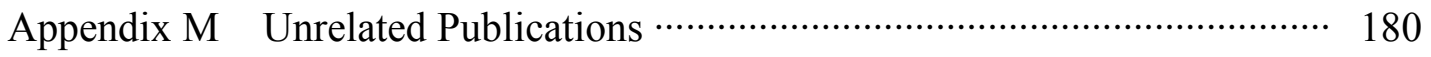

Appendix N List of Publications and Abstracts …….................................... 187 


\section{List of Tables}

\section{Chapter 3 - Manuscript 1}

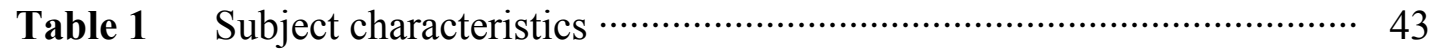

Table 2 Visceral adipose tissue predicted from waist circumference cutoffs associated with increased health risk ………................................ 44

\section{Chapter 4 - Manuscript 2}

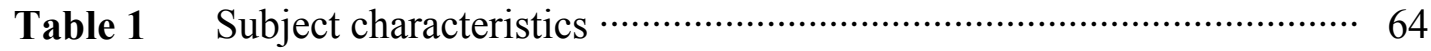

Table 2 The relationships between the VAT and ASAT partial volumes at each inter-vertebral space with the corresponding total VAT and ASAT volume measures ………........................................... 65

\section{Chapter 5 - Manuscript 3}

Table 1 Subject characteristics ……......................................................... 82

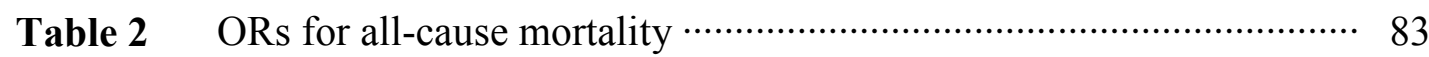

\section{Chapter 6 - Manuscript 4}

Table 1 Baseline Subject Characteristics for the Entire Cohort ……............. 103

Table 2 Baseline Subject Characteristics for the Sub-Sample with CT Data $\cdots 104$

Table 3 Changes in Subject Characteristics in the Sub-Sample with CT Data 105 


\section{List of Figures}

\section{Review of Literature}

Figure 1 Measurement of Liver Fat by Computed Tomography: Normal versus Fatty Liver ……………………………………………... 13

Figure 2 Dose response relationship between reductions in waist circumference and exercise duration in randomized controlled trials 25

Figure 3 Dose response relationship between reductions in visceral AT and abdominal subcutaneous AT with exercise duration in randomized controlled trials

\section{Chapter 3 - Manuscript 1}

Figure 1 Sex differences in the association between waist circumference and total abdominal adipose tissue

Figure 2 Sex differences in the association between waist circumference and abdominal subcutaneous adipose tissue

Figure 3 Sex differences in the association between waist circumference and visceral adipose tissue

\section{Chapter 4 - Manuscript 2}

Figure 1 VAT and ASAT deposition at various measurement sites across the abdomen in 85 men

Figure 2 ORs for prevalent metabolic syndrome using NCEP and IDF criteria according to measurement location for VAT and ASAT in all men and men with a BMI $\geq 27 \mathrm{~kg} / \mathrm{m}^{2}$

\section{Chapter 5 - Manuscript 3}

Figure 1 The OR for mortality with increasing visceral fat mass and area ….

\section{Chapter 6 - Manuscript 4}

Figure 1 Changes in anthropometric variables in response to 6 months of 
Figure 2 Changes in CT Variables in response to 6 months of exercise training 


\section{List of Abbreviations}

\begin{tabular}{|c|c|}
\hline ASAT & Abdominal subcutaneous adipose tissue \\
\hline AT & Adipose tissue \\
\hline BMI & Body mass index \\
\hline CI & Confidence intervals \\
\hline CT & Computed tomography \\
\hline CTL & Liver density assessed by $\mathrm{CT}$ \\
\hline CTS & Spleen density assessed by CT \\
\hline CV & Coefficient variation \\
\hline CVD & Cardiovascular diseases \\
\hline DREW & Dose Response Exercise in Women \\
\hline EMCL & Extramyocellular lipid \\
\hline FFA & Free fatty acids \\
\hline HDL & High density lipoprotein \\
\hline HDM & High density muscle \\
\hline HU & Hounsfield units \\
\hline IDF & International Diabetes Federation \\
\hline IMCL & Intramyocellular lipid \\
\hline IMTG & Intramuscular triglycerides \\
\hline L4-L5 & Intervertebral space between the fourth and fifth lumbar vertebraes \\
\hline LDM & Low density muscle \\
\hline LFS & Liver fat score \\
\hline MetS & Metabolic syndrome \\
\hline MRI & Magnetic resonance imaging \\
\hline MRS & Magnetic resonance spectroscopy \\
\hline NCEP & National Cholesterol Education Program \\
\hline NIH & National Institutes of Health \\
\hline OR & Odds-ratio \\
\hline PAI-1 & Plasminogen activator inhibitor-1 \\
\hline SD & Standard deviation \\
\hline SEE & Standard error of estimate \\
\hline SM & Skeletal muscle \\
\hline
\end{tabular}


T2D Type 2 diabetes

T12-L1 Intervertebral space between the twelfth thoracic and first lumbar vertebraes

TAAT total abdominal adipose tissue

TNF- $\alpha$ Tumor necrosis factor- $\alpha$

TZD Thiazolidinedione

VAT Visceral adipose tissue

$\mathbf{V O}_{2} \mathbf{m a x}$ Maximal oxygen uptake

WC Waist circumference 


\section{Chapter 1. General Introduction}

The prevalence of abdominal obesity worldwide is high and increasing (1-4).

This is a major public health problem as it is now firmly established that abdominal obesity, as commonly assessed using waist circumference, is a strong, independent predictor of morbidity (5-7) and mortality (8). It is suggested that the association between waist circumference and health risk may be explained by its' corresponding association with abdominal subcutaneous and/or visceral adipose tissue (AT) (9). Although the deleterious health outcomes associated with increased abdominal AT are established, there is no consensus definition of visceral AT and abdominal subcutaneous AT, and the potential influence of different measurement protocols on the association between these depots and metabolic risk is unknown. Further, whether abdominal subcutaneous or visceral adiposity is associated with mortality is unknown.

The established association between abdominal obesity and a broad range of health outcomes underscores the need to develop effective therapeutic interventions directed at reducing this AT depot. It is commonly reported that exercise is associated with reductions in abdominal AT (10-14). However, the minimal exercise dose required to elicit a reduction in abdominal obesity is unclear. It is also unknown whether doses of physical activity less than the consensus recommendation for attaining health benefits (ie. 30 minutes on all or most days of the week (15)) are associated with reduction in abdominal obesity, or whether increasing exercise energy expenditure is associated with reductions in abdominal obesity in a dose-response manner.

The aim of the first manuscript of this thesis is to examine the influence of age and gender on the relationship between waist circumference and abdominal AT. The 
second will examine the influence of measurement protocol on the relationship between abdominal AT and metabolic risk while the third considers the independent associations between abdominal subcutaneous and visceral AT with all-cause mortality. The final study will examine the relationship between variations in exercise energy expenditure and corresponding reductions in abdominal AT. The findings from these studies may have important implications in understanding the relationship between abdominal AT with morbidity and mortality, and will contribute to a better understanding of the potential therapeutic benefits of regular exercise. 


\section{Chapter 2. Literature Review}

\subsubsection{Introduction}

Body mass index (BMI) has traditionally been used to characterize obesity and related health risk, and indeed, BMI is related to increased risk for morbidity (16) and mortality (17). The prevalence of obesity worldwide is high and is presently increasing $(1,4)$. In the United States, the prevalence of obesity has increased from $11 \%$ in men and $16 \%$ in women in $1960-1962$, to over $30 \%$ in $1999-2000$ (18). Even more striking is that abdominal obesity, as assessed by waist circumference has also steadily risen from $13 \%$ to $38 \%$ in men and from $19 \%$ to $60 \%$ in women during this same time period (1). Growing evidence suggests that abdominal obesity is a phenotype that is more strongly related to the adverse health outcomes as compared to overall obesity $(9,16)$. Waist circumference is now recognized to be an independent predictor of several metabolic disorders (19-21) even independent of $\operatorname{BMI}(5,22)$.

Physical activity and exercise are associated with decreased morbidity and mortality independent of obesity (23), and are also useful therapeutic strategies for reducing abdominal obesity and visceral adiposity (24). Numerous studies report that waist circumference and visceral adiposity are decreased in response to exercise without caloric restriction, even in the absence of weight loss (24). However, the minimum exercise dose required for eliciting reductions in waist circumference or visceral AT is unclear. Further, the dose response relationship between exercise and reductions in waist circumference or visceral AT is largely unknown.

\subsubsection{Abdominal Obesity and Health Risk}


It is well established that abdominal obesity is a stronger predictor of metabolic risk than lower body or overall obesity $(5,9)$. Waist circumference is commonly used to assess abdominal obesity, and is often a stronger predictor of obesity related metabolic disorders compared to BMI $(5,6,25-28)$. The stronger association between waist circumference and metabolic risk compared to BMI may be partially explained by the ability of waist circumference to predict abdominal obesity. Although BMI and waist circumference have a similar magnitude of association with abdominal subcutaneous AT $(\mathrm{r}=0.52-0.94$ vs. $0.53-0.98)(29-35), \mathrm{BMI}$ tends to be a weaker correlate of visceral AT compared to waist circumference ( $\mathrm{r}=0.41-0.85$ vs. $0.64-0.89)(29-35)$. As visceral AT is reported to be a strong independent predictor of metabolic risk (36-39), the stronger association between waist circumference and visceral AT compared to BMI, may in part explain the stronger association between waist circumference and health risk.

Direct measures of visceral and abdominal subcutaneous AT through imaging techniques are not routinely available in clinical practice. While waist circumference is the single best anthropometric correlate of visceral AT on a population level, the substantial variation in the relationship between abdominal subcutaneous AT and visceral AT limits the ability of waist circumference to predict visceral AT on an individual basis (error $\sim 25-35 \%$ ). In other words, groups with larger waist circumferences will generally have greater amounts of visceral AT as compared to groups with smaller waist circumferences. However, due to the variation in the amount of visceral AT that exists for a given waist circumference between individuals (30-33), it is less likely that a given individual will necessarily have greater amounts of visceral AT compared to another individual with a smaller waist circumference. Similarly, two individuals with the same 
waist circumference could have a two-fold difference in the amount of visceral AT (31). Given the importance of visceral AT in the development of metabolic risk, it is important to examine factors that may influence the association between visceral AT and its surrogate measure, waist circumference. Factors such as age $(40)$, gender $(41,42)$, race $(40,42-44)$ and fitness $(45,46)$ are reported to alter the relationship between BMI and abdominal AT, and may also influence the relationship between waist circumference and abdominal AT. However, as the relationship between BMI and waist circumference itself may also be influenced by these factors $(45,47,48)$, this remains a topic for further investigation. For example, aging is associated with increases in total and visceral adiposity independent of BMI $(40,49,50)$, but there is inconsistency in the literature as to whether this is also true independent of waist circumference. Similarly, it is reported by some that compared to women, men have greater visceral AT for a given BMI, but not waist circumference (51).

\subsubsection{Abdominal Adiposity and Health Risk}

Visceral and abdominal subcutaneous AT are two anatomically and metabolically distinct AT depots. The subcutaneous AT depot lies outside the muscular wall beneath the skin while the visceral AT depot lies within the muscular wall and surrounds the organs. Of the two, visceral AT is more strongly associated with metabolic factors that increase risk for hypertension, dyslipidemia, metabolic syndrome, cardiovascular disease and type 2 diabetes (T2D) (52-57). As compared to subcutaneous adipocytes, visceral adipocytes are much more resistant to the antilipolytic actions of insulin, and therefore have a more sustained elevated free fatty acid (FFA) production (58-61). Furthermore, 
visceral adipocytes release FFAs directly into the portal circulation, which are exposed directly to the liver, unlike subcutaneous adipocytes that only have an indirect FFA delivery to the liver through the systemic circulation (62). As discussed later in this review in further detail, these observations have been used to support a substrate driven theory commonly termed the 'Portal Theory', and has historically been used to explain the association between visceral AT and metabolic risk (62).

Recent evidence demonstrates that AT not only releases FFA into the circulation, but also acts as an endocrine organ secreting numerous other factors that have profound effects on many systems throughout the body ranging from lipid and carbohydrate metabolism, appetite control, blood pressure regulation to the pattern of fat deposition (63-66). The factors released from AT are called adipocytokines, and include leptin, adiponectin, tumor necrosis factor- $\alpha$ (TNF- $\alpha$ ), plasminogen activator inhibitor-1 (PAI-1) and resistin, and are among the most commonly cited secretion products that may be responsible for many of the deleterious metabolic consequences associated with obesity (67-69). The quantity and type of secreted adipocytokines differ between abdominal subcutaneous and visceral adipocytes $(58,60,66)$, and may be responsible for some of the differences in the associations with health risk between the two AT depots. The relationship between these and other cytokines are the topic of several reviews $(68,70$ 77), and thus, only two cytokines of interest will be discussed briefly here.

Adiponectin is an anti-atherogenic factor that is secreted exclusively from adipose tissue (72), and is secreted in higher amounts by visceral (omental) adipocytes as compared to subcutaneous adipocytes (58). In contrast to most cytokines, adiponectin expression is negatively associated with visceral AT and is decreased in obesity $(71,72$, 
75, 78). Adiponectin levels are positively associated with insulin sensitivity (78) but negatively with increased risk for the development of T2D (79) and cardiovascular disease (CVD) (80). It is suggested that the insulin sensitizing effects of adiponectin may be in part attributable to increases in fat oxidation, resulting in decreased skeletal muscle and hepatic lipid infiltration $(71,81)$. Weight loss $(71,78)$ and thiazolidinedione (TZD) $(58,71,81)$, a peroxisome proliferator-activated receptor- $\gamma$ agonist, are two therapeutic strategies associated with increases in adiponectin levels, whereas, TNF- $\alpha$, glucocorticoids, interleukin-6 and beta-adrenergic agonists are associated with decreases in adiponectin (71).

PAI-1 is increased in obesity (82) and decreased with weight loss (83). PAI-1 is a prothrombotic factor that appears to be secreted more abundantly from visceral AT as compared to abdominal subcutaneous AT $(82,84,85)$. PAI-1 inhibits fibrinolysis (82) and is reported to be a predictor of incident T2D independent of fasting glucose, fasting insulin, HDL, triglycerides, hypertension, adiponectin, leptin and visceral AT (79). As with adiponectin, weight loss and TZDs are reported to improve PAI-1 levels.

\subsubsection{Measurement of Abdominal Adiposity}

Although visceral AT is extensively studied in obesity research, there is no consensus as to the anatomical borders or standardized methodology for quantifying this AT depot. Visceral AT can be further sub-divided into the intraperitoneal (within the visceral peritoneum) and extraperatoneal (outside the membrane) AT that differ in their lipolytic activity and blood circulation $(86,87)$. The intraperitoneal (omental and mesenteric) adipocytes are more sensitive to catecholamine-stimulated lipolysis 
adipocytes (88), and drain directly into the portal circulation. The extraperatoneal AT drains into the systemic circulation and surrounds the kidneys, heart and lungs, and also includes AT within the pelvic cavity. Some report that intraperitoneal AT may be more strongly associated with metabolic risk as compared to extraperitoneal AT (58), whereas others do not (55). Whether intra- and extra-peritoneal AT have distinct associations with metabolic risk is currently unclear, and results from imaging studies need to be interpreted with caution as the peritoneum is not visible on CT or MRI images, and the borders of the peritoneal membrane are crudely estimated using other anatomical structures such as the kidneys, aorta and bony structures.

Portally drained visceral adipocytes are reported to lie within the anatomical borders of T10-T11 to L5-S1, with the greatest deposition of omental and mesenteric fat being located in the upper abdomen within the region between L1-L2 and L3-L4 (89). Thus, visceral AT in the upper abdomen may be expected to have a greater impact on hepatic metabolism and metabolic derangements than visceral AT in the lower abdomen. However, visceral and abdominal subcutaneous AT is commonly assessed in the lower abdomen using a single image at L4-L5. L4-L5 was likely selected as the landmark of choice as it was reported to be the best predictor of total body AT (90), not abdominal AT or metabolic risk, and because it is proximal to measures taken at the umbilicus (another common landmark). Interestingly, recent studies report that a single image in the upper abdomen (i.e. L1-L2 or L2-L3) is a better surrogate of total visceral AT $(89,91,92)$ and abdominal subcutaneous AT volume (89) than an image at L4-L5. However, it is still unclear whether the ability to predict the total visceral AT or abdominal subcutaneous AT volume will translate to a corresponding ability to predict metabolic risk. 


\subsubsection{Liver Fat and Health Risk}

Lipid accumulation within the hepatocytes is a novel fat depot that is an independent predictor of insulin resistance (93), hypertension $(54,93,94)$ and dyslipidemia $(54,93-95)$. Some of the earliest work by Kral and colleagues (95), demonstrate that severely obese individuals with fatty liver disease as determined by biopsy, commonly exhibit perturbed hepatic liver metabolism. These initial observations were followed by a series of Finnish studies reporting that liver fat as measured using magnetic resonance spectroscopy (MRS), is a stronger correlate than visceral AT in the independent prediction of health risk $(93,94,96)$. However, for reasons that are unclear, several of these studies report that visceral AT was not a significant predictor of risk (93, 94). Within studies that report both liver fat and visceral AT as significant simple correlates of metabolic risk factors, visceral AT is generally reported to be the stronger predictor of the two fat depots $(21,54,97,98)$. However, this does not detract from the evidence suggesting that both fat depots may be independently related to metabolic disease and as such should be targets of therapeutic intervention.

The mechanisms associated with the development of fatty liver are currently unclear. It is thought that insulin resistance at the level of the subcutaneous adipocyte, or an enlarged visceral AT depot of inherently insulin resistant adipocytes may lie at the center of fatty liver disease (99-101). Insulin resistant adipocytes have an increased FFA release due to the diminished ability of insulin to inhibit lipolysis (61), and the FFAs from the visceral depot are delivered directly to the liver via the portal circulation, in turn causing an increased production of triglycerides and very low density lipoproteins. 
According to the portal theory, an enlarged visceral AT depot that delivers a FFA flux that surpasses the ability of the liver to secrete or oxidize the lipid results in lipid accumulation within the hepatocytes (62). Increased lipid accumulation within the hepatocytes or steatosis is associated with impaired hepatic glucose metabolism, and reduced insulin clearance, which results in increased gluconeogenesis and hyperinsulinenia $(21,62,97,102-104)$. Chronically elevated circulating insulin and glucose further contributes to this negative cycle leading to peripheral skeletal muscle insulin resistance and possibly T2D. However, this theory is difficult to substantiate in humans due to the inaccessibility of the portal vein. Indirect methods estimate the contribution of FFA from the visceral AT depot only accounts for 5 to $20 \%$ of the FFA flux to the liver (105). As such, an enlarged subcutaneous AT depot may be a more important contributor to the FFA flux to liver, and the consequent development of fatty liver. However, this is in conflict with the majority of cross-sectional data that suggest that liver fat may be more closely associated with visceral AT as compared to subcutaneous AT $(21,54,96,106,107)$.

An emerging theory suggests that all ectopic fat deposition may have a similar etiology, and that an increased capacity to buffer energy and store lipid in the glutealfemoral subcutaneous adipocytes may 'protect' against excess lipid deposition within tissues ectopic to subcutaneous fat such as the liver, muscle and visceral AT (108). Unlike gluteal-femoral subcutaneous adipocytes, excess lipid accumulation within these ectopic depots is clearly associated with deleterious metabolic consequences $(21,54,93$, $98,99,109-112)$. This is illustrated in individuals with lipodystrophy $(113,114)$ or in aging $(115,116)$; two conditions that are associated with minimal subcutaneous AT, but 
increased ectopic fat deposition in the liver and muscle, visceral adiposity and accordingly, a marked unfavorable metabolic profile. Conversely, individuals with multiple symmetric lipomatosis are associated with excessive subcutaneous AT deposition, but with minimal lipid accumulation in the liver, muscle and visceral AT, and a normal metabolic profile (117).

In addition to endogenous FFA from AT stores, increased FFA delivery to the liver can also result from increased exogenous FFA sources. Increased dietary fat consumption is also associated with increased fatty infiltration of the liver (118-120), and may also result in deleterious metabolic consequences. It is unclear whether the type of dietary fat (ie. saturated or unsaturated) influences liver fat accretion and whether fatty liver resulting from exogenous FFA delivery is associated with the same metabolic consequences as fatty liver resulting from endogenous FFA.

There are also reports that fatty liver may be associated with cytokines secreted from the adipocytes. Adiponectin is reported to reduce FFA turnover in the liver (121), and is negatively associated with liver fat (101). Drug therapies, such as TZDs, that are associated with increases in adiponectin are also associated with decreases in hepatic fat deposition $(81,122-124)$. Interestingly, this class of drug is also associated with increases in subcutaneous adipocytes proliferation and decreases in visceral AT (125, 126). Currently, the relative contributions of FFA delivery, adiponectin (FFA turnover) or other factors in the development of fatty liver remain unclear.

\subsubsection{Measurement of Liver Fat}


Several methods are currently employed to measure liver fat. Three common methods in obesity research include histology, MRS and CT. Of these the liver biopsy is considered the gold standard (127) for assessing liver disease as it is the only method available for assessing liver conditions such as inflammation, fibrosis and cirrhosis (128130), in addition to liver steatosis (fat). However, for clinical studies it would be unethical to routinely take liver biopsies for measurement of liver fat as it may be painful, and is associated with risk of bleeding and other complications that may result in hospitalization and possible mortality (131). Values from CT $(r=0.77$ to 0.90$)$ and MRS $(\mathrm{r}=0.68$ to 0.90$)$ techniques are generally well correlated to those from liver biopsies and may provide a safer non-invasive alternative $(129,132-139)$.

CT is used to measure hepatic fat infiltration by determining the attenuation value for each voxel within a region of interest in the liver. The $\mathrm{CT}$ attenuation value is dependent upon the molecular composition of the tissues within each voxel. Lipid has a lower density than water and protein, and increasing liver fat infiltration is reflected by a lower liver density and thus lower attenuation values (140). However, there is an overlap between the ranges of normal and fatty liver values (130). As such, it has been suggested that the liver density (CTL) should be expressed relative to the spleen attenuation value (CTS) (Figure 1) (130). In normal individuals, the liver and spleen attenuation values have a constant relationship wherein the liver is a denser organ and thus has a higher attenuation value. Therefore CTL/CTS less than one is indicative of fatty infiltration $(130,140)$. Normally, the liver and spleen mean attenuation values are derived from two or three regions of interest within the liver and spleen. However, due to the small area of interest, and subjectivity involved in placing the regions of interest, we have recently 
Figure 1: Measurement of Liver Fat by Computed Tomography: Normal versus Fatty Liver

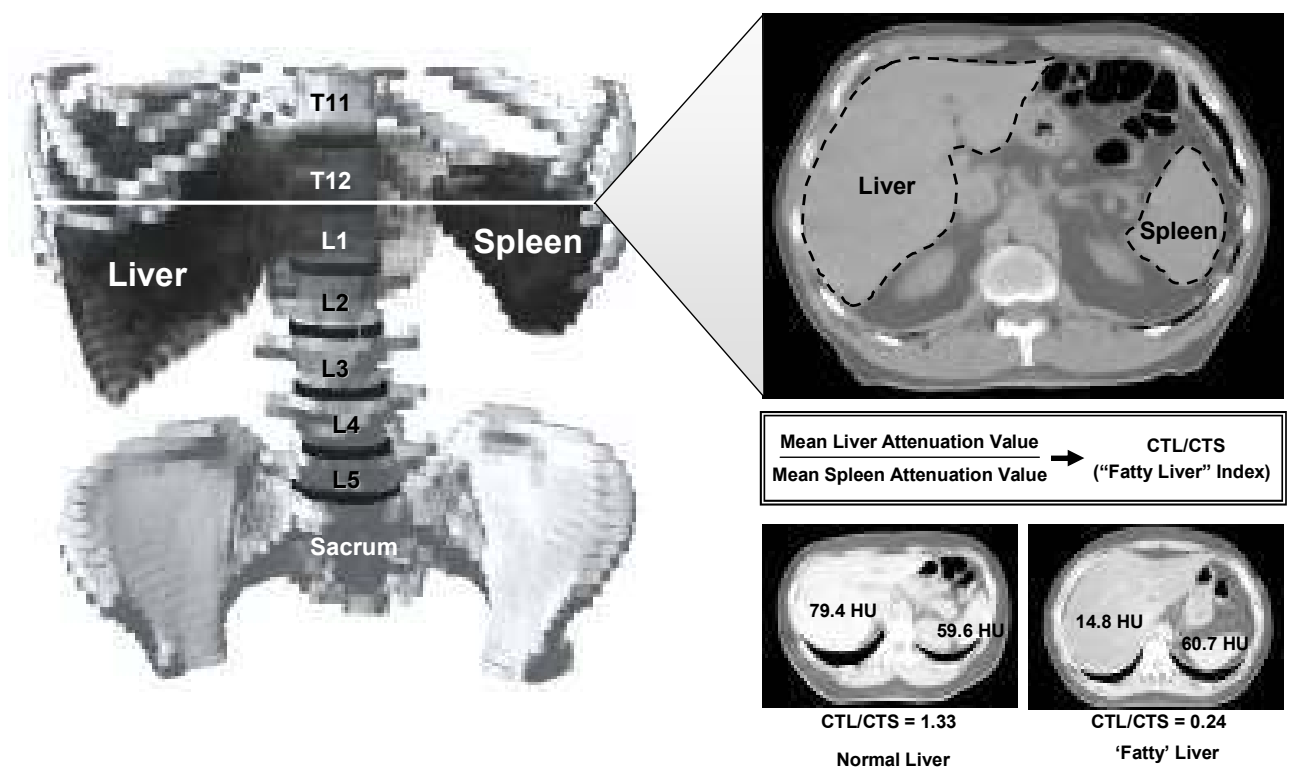


suggested that the whole liver and spleen surface areas should be used to derive the respective mean attenuation values (Figure 1) (141). Contrary to previous reports (21), we demonstrate that the attenuation values within the liver and spleen are fairly homogeneous throughout, and by using the whole surface area the inter-observer coefficient of variation in the analyses is slightly reduced from $5.1 \%$ (54) to $2.9 \%$ (141). However, obtaining a CT image that contains both liver and spleen presents a challenge; not only does variation exist in the vertical positioning of the spleen relative to the liver, but in positioning of both organs within the abdominal cavity. As a multi-image approach is not feasible due to excess exposure (142), Davidson et al. (141) proposed that a single axial image at the T12-L1 inter-vertebral space may provide the most optimal landmark for assessing both liver and spleen attenuation, as liver and spleen were identified at that level in approximately $90 \%$ of the men and women studied (141)

\section{(Figure 1).}

Unlike $\mathrm{CT}$, proton magnetic resonance spectroscopy $\left(\mathrm{H}^{1} \mathrm{MRS}\right)$ is not associated with radiation exposure, and provides a quantitative assessment of liver fat within a region of interest or voxel $\left(2\right.$ to $\left.3 \mathrm{~cm}^{2}\right)$ placed in the parenchyma of the right lobe of the liver. MRI facilitates this process and ensures accurate and consistent landmarking, and ensures the avoidance of major blood vessels, and areas of inhomogeneity within the liver. Briefly, MRS uses magnetic fields to deviate hydrogen protons from their normal orbits. When the protons relax back to the normal orbit, there is an energy release and the generation of radio frequency signals. $\mathrm{H}^{1}$ MRS uses the differing proton resonance signals from the fatty acyl groups (143-146) to generate a peak that reflects the intracellular component (methelyene signal) measured at 0 to $3.0 \mathrm{ppm}$, and another 
proton signal from water to generate a larger peak between 3.0 and $7.8 \mathrm{ppm}$ (129). The percent liver fat is derived from the ratio of the area under the curve for fat is then compared relative to the area under the curve for water $(93,129,139)$. 


\subsubsection{Skeletal Muscle Lipid and Health Risk}

Skeletal muscle is reported to be responsible for $90 \%$ of insulin stimulated glucose uptake (147). Thus, metabolic derangements caused by lipotoxicity at the level of the myocyte are thought to be involved in the development of insulin resistance and T2D. Pan et al. (148) made one of the initial observations relating intramuscular triglycerides (IMTG) with insulin resistance, a finding that has since been replicated by several others (149-152). As with liver fat, it is suggested that muscle lipid also accumulates as a function of increased FFA flux from either endogenous or exogenous sources. The importance of muscle lipid deposition in the development of insulin resistance was demonstrated by Bachmann et al. (149) who report that elevations in FFA through infusion (6 hours) or a high fat diet (3 days) are associated with increases in IMTG and accordingly insulin resistance. Due to the short duration of these studies, the interventions probably did not influence visceral fat deposition, but may have increased liver fat, which is another dynamic fat depot that is sensitive to deviations in energy balance. However as liver fat was not assessed, these results cannot exclude liver fat deposition in the etiology of insulin resistance. There is also a paradoxical observation by Goodpaster et al. (153) who report that athletes have IMTG levels comparable to obese or individuals with T2D, but are insulin sensitive. The authors in that study proposed therefore, that metabolic differences in muscle lipid kinetics may explain the differences in insulin sensitivity observed beyond simply the quantity of lipid present.

\subsubsection{Measurement of Skeletal Muscle Lipid}


As with liver fat, the average $\mathrm{HU}$ or mean attenuation value for adipose tissuefree skeletal muscle voxels can be used as an index of skeletal muscle lipid content (109). Thus, the lower the skeletal muscle mean attenuation value, or the greater the number of low-density skeletal muscle voxels (e.g., 0-30 HU), the higher the skeletal muscle lipid content. However, it is important to note that unlike liver, muscle attenuation values by $\mathrm{CT}$ are a reflection of both intra-myocellular and extra-myocellular lipid (IMCL and EMCL, respectively) content, and although correlated, they are not analogous to intramyocellular lipid values obtained by skeletal muscle biopsy or proton magnetic resonance spectroscopy.

Schick et al. (146) were the first to identify the two compartments of triglycerides in muscle that are located inside (IMCL) and outside (EMCL) the muscle cell. IMCL droplets are spherical with a relatively homogenous distribution, and as such, are independent of muscle orientation relative to the magnetic field $(143-145,154)$. On the other hand, EMCL lies outside of the muscle fibre and appears in plate-like structures $(143-145,155)$ that have a highly variable distribution throughout the muscle. Due to the varying orientations and patterns of EMCL, misalignment of the muscle fibres relative to the magnetic field will cause the EMCL line to broaden, and potentially overlap with the IMCL resonance peak (145). Boesch et al. demonstrated that IMCL, and not EMCL signals scale linearly with voxel size, water and creatine signals which are used to quantify and scale the IMCL spectra $(143,144)$. As such, quantification of IMCL using MRS is much more repeatable (CV 6\%) (143-145) than measures of EMCL (CV 53\%) (145). Presently there is no available method for accurately quantifying EMCL. 
Consequently, the metabolic significance of EMCL has yet to be clearly demonstrated in a well-controlled study, but is generally thought to be metabolically inert (155).

\subsubsection{Influence of Physical Activity and Exercise on Obesity and Health Risk}

It is widely reported that engaging in physical activity or having a higher cardiorespiratory fitness is associated with reduced risk for morbidity and mortality (23, $156,157)$. While the principal mechanisms by which exercise is associated with improvements in metabolic profile or risk for morbidity and morality are unclear, several plausible mechanisms exist. These range from the acute benefits associated with a single bout of aerobic physical activity on lipid or glucose metabolism $(158,159)$, to more gradual changes associated with regularly repeated bouts of physical activity including improvements in cardiopulmonary function (160), cardiovascular/circulatory system (160) or changes in body composition $(11,12,161,162)$. In particular, chronic physical activity has been shown to significantly reduce abdominal obesity, specifically visceral $\operatorname{AT}(11,12,162)$.

\subsubsection{Influence of Physical Activity and Exercise on Obesity}

It is clear that exercise is a useful strategy for inducing reductions in body mass and adiposity through increases in energy expenditure resulting in negative energy balance $(11,12)$. However, there is also evidence that individuals who engage in regular physical activity are phenotypically different from individuals with low cardiorespiratory fitness despite having a similar BMI or total adiposity $(45,46)$. For a given BMI, individuals with high cardiorespiratory fitness have lower levels of visceral and 
abdominal subcutaneous adiposity as compared to individuals with low cardiorespiratory fitness (45). For example, a 50 year old man with high cardiorespiratory fitness (upper four quintiles) and a BMI of 25 to $27 \mathrm{~kg} / \mathrm{m}^{2}$ would be expected to have approximately 10 to $16 \%$ less visceral AT than an individual of the same age and BMI in the lowest quintile of cardiorespiratory fitness (45). Thus, reduced visceral AT is a plausible mechanism by which high cardiorespiratory fitness levels may attenuate the health risk associated with a given degree of obesity.

Several exercise training studies also corroborate these findings, and report that regular physical activity is associated with improvements in body composition independent of weight loss $(11,12,163)$. For example, Ross et al. $(11,12)$ demonstrate that regular moderate intensity exercise is associated with substantive reductions in visceral and abdominal subcutaneous AT despite an absence of changes in weight in both middle-aged obese men and women. Similarly, Thomas et al. (164) report that even nonobese premenopausal women with relatively low levels of visceral AT experience a significant $25 \%$ reduction in visceral AT in response to 6 months of aerobic exercise despite no significant change in weight.

Although available data would clearly support the notion that regular aerobic exercise is associated with reductions in visceral adiposity independent of weight loss, it is important to note that exercisers who lose weight have substantially greater reductions in visceral AT compared to exercisers who maintain body weight $(11,12)$. Thus, from a clinical perspective exercise-induced weight loss should be advocated to those at risk for obesity-related metabolic disorders, as it is likely associated with the greatest benefit. However, given the challenges associated with attaining substantial weight loss for many 
obese individuals, it is equally important to appreciate that abdominal obesity and related health risk may be markedly reduced in response to minimal amounts of weight loss.

\subsubsection{Influence of Physical Activity and Exercise on Health Risk}

The weighted evidence suggests that acute exercise bouts are associated with increased HDL, decreased triglycerides (158) and improvements in insulin sensitivity $(159,165)$. Improvements in HDL and triglycerides appear to be related to the energy expenditure $(158,166)$, and thus acute effects of a single exercise bout may be more apparent in trained individuals who are capable of greater energy expenditures (158). These effects are transient, lasting up to 72 hours and are independent of changes in cardiorespiratory fitness. Similarly, improvements in insulin sensitivity last 48 to 72 hours (159) and even a single bout of moderate intensity exercise is associated with a substantial $25 \%$ improvement in insulin sensitivity in both normal individuals, and in offspring T2D parents (165). Accordingly, regular bouts of physical activity may induce a beneficial health effect independent of changes in obesity.

Several studies report a beneficial effect of cardiorespiratory fitness or physical activity even independent of BMI $(23,156)$ and overall adiposity $(156)$. Wei et al. (23) report that individuals with low cardiorespiratory fitness who were classified as normal weight by BMI had twice the mortality risk as compared to obese individuals with high cardiorespiratory fitness. Similarly, Lee et al. (156) report that when men were categorized by percent body fat $(<16.7 \%)$ or waist circumference $(<87 \mathrm{~cm})$, lean men with low cardiorespiratory fitness had a higher mortality risk than obese men $(>25 \%$ body fat or waist circumference $>99 \mathrm{~cm}$ ) with high cardiorespiratory fitness. Further, 
cross-sectional data from Lee et al. (167) demonstrate that men with a high cardiorespiratory fitness have a lower prevalence of the metabolic syndrome independent of visceral and abdominal subcutaneous AT. These observations reinforce the importance of having a high cardiorespiratory fitness, but should not detract from the importance of maintaining a normal body weight. In all the aforementioned studies, there was also a clear association between obesity and mortality risk.

Many studies report that changes in metabolic risk are more closely related to changes in adiposity as opposed to changes in fitness or physical activity. Katzsel et al. (168) examined the effect of a $10 \%$ reduction in body as compared to a $10 \%$ improvement in cardiorespiratory fitness to improve the lipid profile. Weight reduction was achieved through caloric restriction, and the improvement in cardiorespiratory fitness was achieved through exercising three times a week while maintaining a stable body weight over a nine month period. As expected, the diet weight loss group did not alter their cardiorespiratory fitness, but significantly reduced body fat. Conversely, the exercise group significantly improved their cardiorespiratory fitness, but did not significantly reduce body fat. In that study, only the diet weight loss group report significant improvements in the lipid profile, and as such, the authors of that study concluded that weight loss is more beneficial for improving CVD risk factors as compared to increasing cardiorespiratory fitness. Similarly, Katzmarzyk et al. (169) suggest that changes in lipids consequent to exercise training is more closely related to changes in fatness than cardiorespiratory fitness.

These observations should not detract from the importance of engaging in physical activity. Indeed, for a given amount of weight loss, exercise is associated with 
greater improvements reductions in adiposity, a better maintenance of lean body mass, and improvements in cardiorespiratory fitness as compared to caloric restriction in men and women $(11,12,163)$. As such, it appears that pound for pound, exercise may be a better treatment strategy for improving health and obesity reduction. Moreover, exercise without weight loss is associated with significant reductions in waist circumference, and total and visceral adiposity $(11,12,163)$, and improvements in insulin sensitivity $(159$, 165). Although exercise without weight loss is not as beneficial as exercise with weight loss, one should not consider exercise in the absence of weight loss a failure for decreasing obesity-related health risk.

\subsubsection{Dose Response Relationship between Exercise and Obesity Reduction}

There are currently several exercise recommendations for attaining health benefits ranging from as little as 20 minutes, 3 or more times a week to as much as 60 minutes of daily moderate intensity physical activity $(15,170-172)$. However, based on the limited data available from randomized controlled trials, the minimal amount of exercise required to elicit a reduction in abdominal obesity is largely unknown. Whereas some randomized controlled trials report significant reductions in waist circumference (173-175), some do not $(168,176,177)$. The discrepant findings may be partially explained by small sample size $(176,177)$ or increased dietary intake to maintain body weight $(168)$. Studies that prescribe $\sim 150$ minutes/week of moderate intensity exercise generally report a 1 to $3 \mathrm{~cm}$ reduction in waist circumference relative to the respective control group (173-175). However, studies that prescribe exercise doses of less than the recommendation $(\sim 100$ minutes/week) also show reductions in waist circumference in the order of $2 \mathrm{~cm}(178$, 
179), whereas the few studies that prescribe more than the recommendations $(\sim 450$ minutes/week) show substantially larger reductions in waist circumference in the order of $6 \mathrm{~cm}(11,12)$.

From this data there appears to be a very modest dose response relationship between weekly physical activity duration and reductions in both waist circumference (Figure 2) and abdominal adiposity (visceral and abdominal subcutaneous AT, Figure 3). In general, moderate physical activity of $\sim 150$ to 200 minutes per week is associated with modest reductions in waist circumference $(\leq 3 \mathrm{~cm})$ and abdominal adiposity $(5-10 \%)$ as compared to their respective control groups, and increasing daily physical activity to approximately 450 minutes per week is associated with a $\sim 30 \%$ and $\sim 20 \%$ reduction in visceral and abdominal subcutaneous AT, respectively. However, these studies differed in terms of the exercise intensity (40 to $80 \% \mathrm{VO}_{2 \max }$ ), length of the intervention (2 to 12 months), study cohort characteristics (men vs women, younger vs older, T2D vs no T2D, etc.), exercise modality (walking, biking, etc.) or controlling dietary intake $(168,174)$; all of which would influence the exercise energy expenditure, net energy balance and ultimately the expected reduction in waist circumference. Accordingly, it is difficult to infer a dose response relationship between exercise and reductions in abdominal adiposity from the current literature.

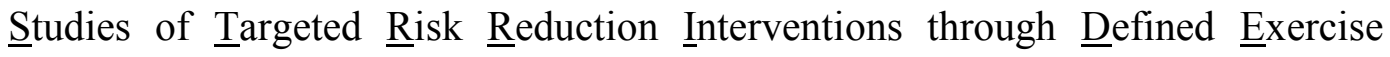
(STRRIDE) $(174,180,181)$ is one of the few studies that have examined the doseresponse relationship between exercise with obesity reduction and improvements in health risk. As expected, they report that obese middle-aged individuals who exercised for about $175 \mathrm{~min} / \mathrm{wk}$ lost significantly more body weight and total fat mass than those 
who exercised about $114 \mathrm{~min} / \mathrm{wk}$ (174). However, reductions in total abdominal, visceral and abdominal subcutaneous AT were significant only among those who exercised for $175 \mathrm{~min} / \mathrm{wk}$, although both the $175 \mathrm{~min} / \mathrm{wk}$ and the $114 \mathrm{~min} / \mathrm{wk}$ of physical activity groups tended to reduce their WC (174). The authors of that study did not report if there is a dose response relationship between exercise duration and reductions in abdominal obesity. Further, these results must be interpreted with caution as the participants in the trial were instructed to maintain their baseline body weight by increasing their caloric intake. The compensatory consumption of additional calories would lead to a significant underestimation of the effects of exercise per se on body weight and fat mass, thereby confounding interpretation. The study by Irwin et al. (175) is one of a few that report a dose-response effect of exercise volume $(\leq 135,136-195$ or $>195 \mathrm{~min} /$ week $)$ on reductions in total and visceral adiposity. However, this was a retrospective analysis of mainly self-report data, and actual energy expenditure and changes in waist circumference were not determined. As such, the dose response relationship between exercise volume and reductions in abdominal obesity requires further investigation. 
Figure 2: Dose response relationship between reductions in waist circumference and exercise duration in randomized controlled trials

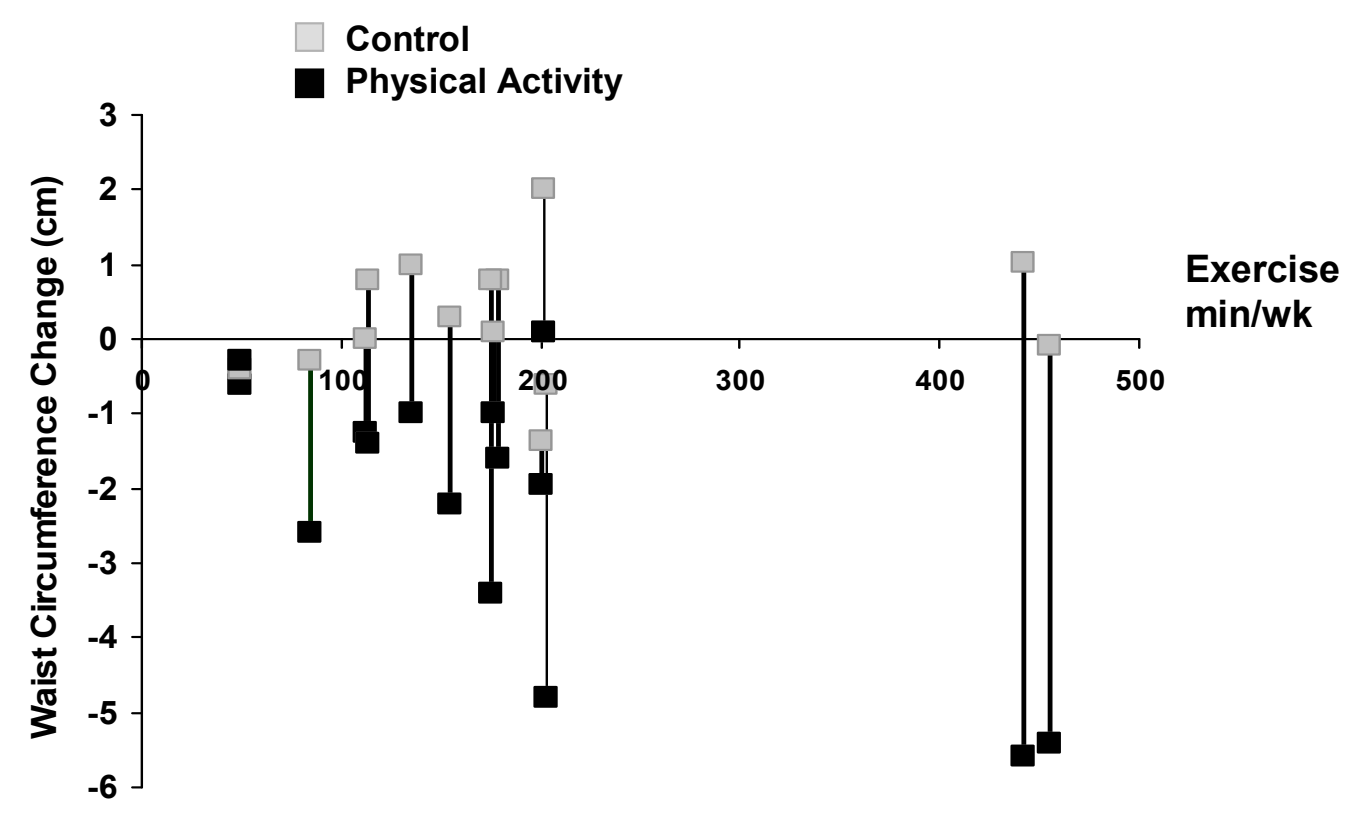

References $(11,12,168,173-178,182-184)$ 
Figure 3: Dose response relationship between reductions in visceral AT and abdominal subcutaneous AT with exercise duration in randomized controlled trials
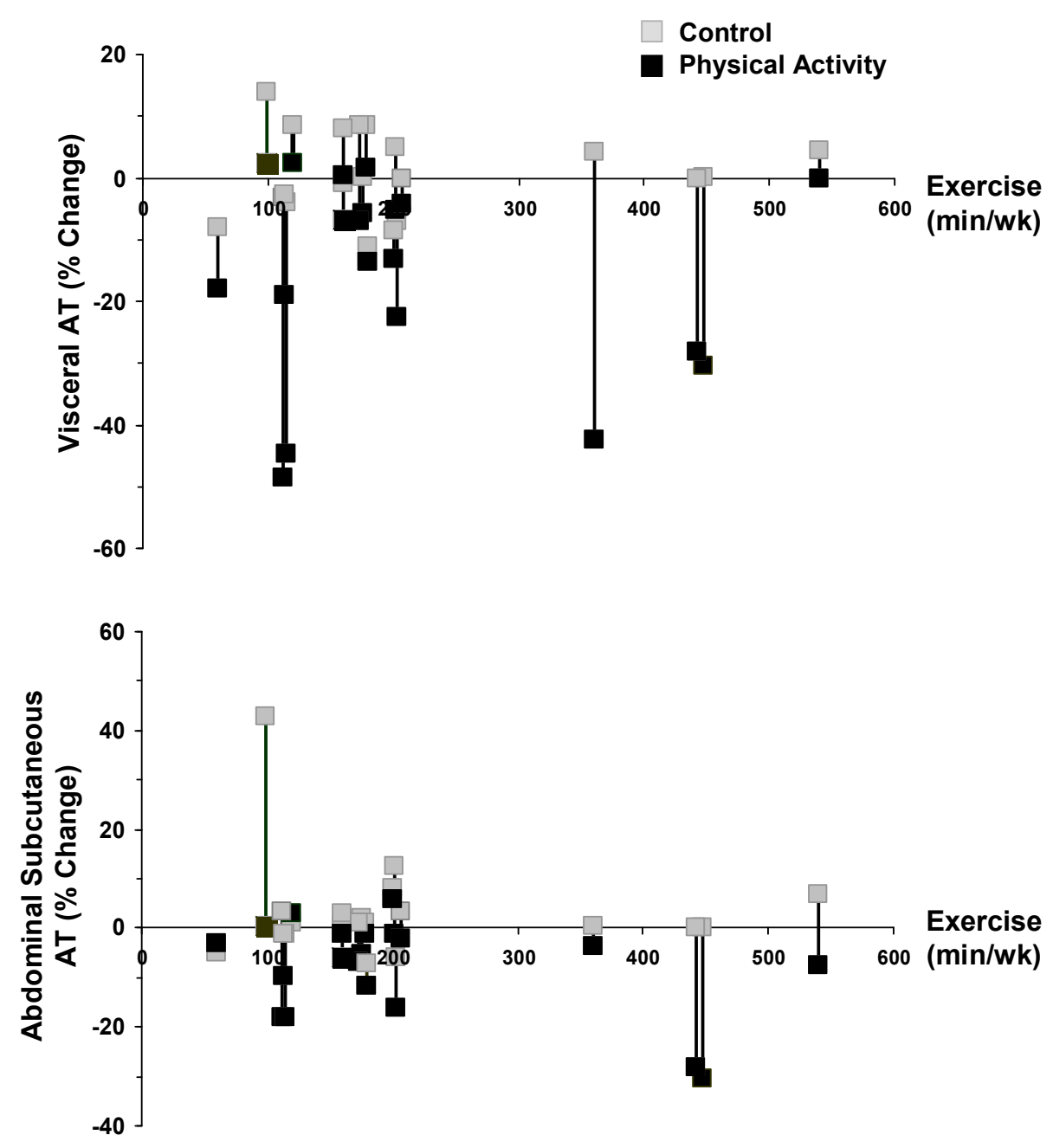

References $(11,12,161,162,174-177,185-191)$ 


\subsubsection{Summary}

The dramatic rise in the prevalence of abdominal obesity and obesity-related metabolic disorders necessitates the need for studies that further examine the association between abdominal obesity and health risk, and therapeutic strategies designed to reduce waist circumference, and sets the stage for the present thesis. The aim of the first manuscript is to examine the influence of age and gender on the relationship between waist circumference and abdominal AT. The second will examine the influence of measurement methodology on the relationship between abdominal AT and metabolic risk. The independent associations between abdominal subcutaneous and visceral AT with allcause mortality will be examined within the third study and the final will examine the relationship between exercise energy expenditure and reductions in abdominal AT.

The findings from these studies may have important implications for understanding the relationship between abdominal AT, morbidity and mortality, and will contribute to a better understanding of the potential therapeutic effects of regular exercise. 


\section{Chapter 3. Manuscript 1}

This manuscript has been published in The American Journal of Clinical

Nutrition. The co-authors of this manuscript are SoJung Lee, Steven B. Heymsfield and Robert Ross. This research was supported in part by research grants from the Canadian Institutes of Health Research to Robert Ross (MT13448) and the National Institutes of Health to Steven B. Heymsfield (RR-00645 and DK-42618). 
Waist circumference and abdominal adipose tissue distribution:

Influence of age and gender 


\section{ABSTRACT}

Background: The influence of age and gender on the distribution of abdominal adipose tissue for a given waist circumference (WC) is unclear.

Objective: To investigate the influence of age and gender on total abdominal (TAAT), visceral (VAT) and abdominal subcutaneous adipose tissue (ASAT) for a given WC. Design: Subjects included 147 younger men (age $<50$ yr), 83 older men, 171 younger (premenopausal) women and 80 older (postmenopausal) women with a wide range in BMI (16-40 kg/m²). Body composition was assessed using a whole body magnetic resonance protocol.

Results: Within each gender, the regression lines between WC and TAAT were not significantly different $(P>0.1)$ between younger and older groups. Collapsed across age groups, women had more TAAT for a given WC than men, however, this difference was significantly reduced with increasing WC $(P<0.05)$. Within each gender, regression lines derived for WC and ASAT were not significantly different between younger and older groups $(P>0.1)$. Collapsed across age groups, women had $1.8 \mathrm{~kg}$ more ASAT for a given WC $(P<0.05)$ than men across the range of WC. Within each gender, older men and women had a significantly greater increase in VAT for a given WC $(P<0.05)$ than younger men and women. Further, independent of age group, the slope for WC and VAT was significantly higher $(P<0.05)$ in men than women.

Conclusions: There are significant gender differences in TAAT, VAT and ASAT for a given WC. Further, the relationship between WC and VAT is substantially influenced by age. 
Keywords: Visceral fat, abdominal subcutaneous fat, gender, menopause, age, magnetic resonance imaging 


\section{INTRODUCTION}

Previous studies have reported that for a given waist circumference, premenopausal (51) and postmenopausal (41) women have more abdominal subcutaneous adipose tissue (AT) than men. However, it is unclear how gender alters the association between visceral AT accumulation and waist circumference. Pouliot et al. (51) suggest that men and women have similar amounts of visceral AT for a given waist circumference. In contrast, Schreiner et al. (41) report that although older men generally have more visceral AT than older women, this difference is diminished with increasing waist circumference. Whether the disparate findings between these studies are explained by differences in age is unknown.

It is well known that age is positively associated with an increase in visceral AT independent of gender $(50,192)$. The age-related increase in visceral AT is greater in men than women, however, in women visceral AT accumulation increases rapidly after menopause to a rate similar to that of men (192). In contrast, subcutaneous AT has been reported to decrease with age in men (192). Whether this remains true in women is unclear as some studies report an age related decrease in abdominal subcutaneous AT (50), whereas others do not (192). Accordingly, age associated changes in abdominal AT distribution are not always reflected by a change in total abdominal AT or waist circumference $(50)$.

Waist circumference is a strong predictor of both visceral and abdominal subcutaneous AT (29). Given the independent contributions of visceral (193) and subcutaneous AT (52) in the development of metabolic risk, it is important to understand the influence of gender and age on abdominal AT distribution and its surrogate measure, 
waist circumference. Thus, the purpose of this study was to examine the influence of gender and age on the relationship between waist circumference and abdominal AT accumulation in a large sample of Caucasian men and women with a wide range of age and adiposity. We hypothesized that advanced age would be associated with greater visceral AT deposition for a given waist circumference independent of gender. Further, we speculated that men would have greater visceral AT and less abdominal subcutaneous AT than women for a given waist circumference. 


\section{RESEARCH DESIGN AND METHODS}

\section{Subjects}

Subjects consisted of 483 healthy Caucasian men $(n=232)$, premenopausal $(n=$ $171)$ and postmenopausal women $(n=80)$ who participated in various body composition studies at Queen's University (Kingston, Canada) and St. Luke’s-Roosevelt Hospital (New York, NY). The subjects varied widely in age (18-84 yr) and BMI $\left(16-40 \mathrm{~kg} / \mathrm{m}^{2}\right)$. Three hundred and forty-five subjects from Queen's University and 136 subjects from Columbia University were recruited from among hospital employees, students at local universities, and the general public through posted flyers and the local media. Menopausal status was determined by self-report. The men were divided into younger $(n=147)$ and older $(n=83)$ by using a cut-off age of 50 years to facilitate comparisons between older men and (postmenopausal) women, and younger men and (premenopausal) women across a similar age range. All participants gave informed consent before participation in accordance with the ethical guidelines of the respective institutional review boards.

\section{Anthropometric Measurements}

Body mass was measured to the nearest $0.1 \mathrm{~kg}$ on a calibrated balance. Standing height was measured to the nearest $0.1 \mathrm{~cm}$ using a wall-mounted stadiometer. Waist circumference was taken at the level of the last rib to the nearest $0.1 \mathrm{~cm}$ after a normal expiration. 


\section{Measurement by Magnetic Resonance Imaging}

Whole body tissue quantification was determined using magnetic resonance imaging data acquired with a General Electric 1.5-Tesla magnet (Milwaukee, Wisconsin) by using an established protocol described in detail elsewhere (194). Once acquired, the data was analyzed with an image analysis program (TomoVision, Montreal, QC, Canada) as described previously $(194,195)$. Approximately 41 equidistant images (1-cm-thick images, 4-cm spaces between images) were used to determine the tissue volumes. Visceral AT and abdominal subcutaneous AT were calculated using the five images extending from $5 \mathrm{~cm}$ below to $15 \mathrm{~cm}$ above L4-L5. Volume units (L) were converted to mass units (kg) by multiplying the volumes by the assumed density constants for AT $(0.92 \mathrm{~kg} / \mathrm{L})(196)$.

\section{Statistical Analyses}

Group data are presented as group means $\pm \mathrm{SD}$. ANOVA was used to determine group differences in subject characteristics. Bonferroni adjustments were used for all post hoc multiple group comparisons. General linear modeling was used to determine group-specific multivariate regressions between the various study variables. Gender, age, waist circumference and the interaction effects were used in the multiple regression analyses. Quadratic terms were also tested in the model for waist circumference. Models were tested first for significant age group, gender and waist circumference interactions. If the 3-factor interaction effect was significant, further analyses were conducted within each gender separately. If the 3-factor and age interaction terms were not significant, a single model was used to determine gender differences in slope and intercept collapsed 
across age group. The study variables were normally distributed and residual analyses were performed to determine significant points of influence in the final models. Significant outliers with large jackknife residuals and high leverage were excluded from the analyses. To allow for comparisons between premenopausal and postmenopausal women, figures were age standardized to 50 year of age. To allow for comparisons in the younger men and women, figures and tables were standardized to 25 - and 40- year old men and women. All statistical procedures were performed using SAS version 8.2. 


\section{RESULTS}

Subject characteristics are presented in Table 1. BMI was not significantly different $(P>0.05)$ between any of the four groups. As expected, both groups of women had a significantly higher $(P<0.05)$ body fat percentage, but a lower waist circumference and less visceral AT than the men. While the older men had a significantly higher $(P$ $<0.05$ ) waist circumference and more visceral AT than the younger men, the younger and older women were not different in any of the body composition measures.

Age was positively associated with waist circumference, visceral AT, and abdominal subcutaneous AT in only the younger groups of men and women (data not shown). Therefore, statistical adjustment for age was restricted to gender comparisons between the younger men and women.

The 3-factor interaction (age*gender*WC) effect was not significant $(\mathrm{P}>0.05)$. However, there was a significant gender, but not age group interaction effect $(\mathrm{P}>0.05)$ on the relationship between waist circumference and total abdominal AT $(\mathrm{P}<0.05)$. Collapsed across the age groups, women had more abdominal AT for a given waist circumference than men, however, this difference was significantly reduced $(P<0.05)$ with increasing waist circumference (Figure 1). Age was not a significant covariate ( $P$ $>0.05$ ) in any of the models for waist circumference and abdominal AT.

The 3-factor interaction (age*gender*WC) effect was not significant $(\mathrm{P}>0.05)$. There was a significant gender, but not age group interaction effect $(\mathrm{P}>0.05)$ on the relationship between waist circumference and abdominal subcutaneous AT $(\mathrm{P}<0.05)$. Collapsed across the age groups, women had $1.8 \mathrm{~kg}$ more $(P>0.05)$ abdominal 
subcutaneous AT for a given waist circumference than men across the range of waist circumference values (Figure 2).

There was a significant 3-factor interaction (age*gender*WC) effect $(\mathrm{P}<0.05)$, and thus visceral AT analyses were performed within each gender separately. Within each gender, the slope of the regression lines between waist circumference and visceral AT were significantly higher in the older men and women than the younger men and women $(\mathrm{P}<0.05)$. Thus for a given increase in waist circumference, men have a greater increase in visceral AT than women. Further, independent of age group, the slope of the regression line between waist circumference and visceral AT was higher $(P<0.05)$ in men than women (Figure 3a+b). After including age in the model, younger men continued to have a higher slope for the association between waist circumference and visceral AT than younger women (Figure 3c).

The postmenopausal women continued to have a greater increase $(\mathrm{P}<0.05)$ in visceral AT for a given increase in waist circumference (i.e. higher slope) after control for age, and the difference increased with increasing waist circumference. The relationship between waist circumference and visceral AT standardized to 50 years of age is shown in Figure 3d.

The predicted visceral AT mass values for waist circumference cut-points associated with increased health risk in men and women are given in Table 2. Given the common use of the L4-L5 landmark for determination of abdominal AT deposition using radiographic methods, visceral AT values $\left(\mathrm{cm}^{2}\right)$ at L4-L5 are also provided. 


\section{DISCUSSION}

The findings from this study demonstrate that significant gender differences exist in total abdominal, visceral and abdominal subcutaneous AT deposition for a given waist circumference. Although our findings confirm that women have significantly more abdominal subcutaneous AT than men for a given waist circumference, in contrast to others, we observed that visceral AT deposition was significantly higher in men than women. Further, we demonstrate that menopausal status influences visceral AT deposition for a given waist circumference beyond that of age per se. Moreover, the influence of age on visceral AT deposition is different between older and younger individuals. These findings suggest that for a given waist circumference, gender, age and menopausal status need to be considered when predicting abdominal AT distribution and associated health risk.

Contrary to previous observations $(41,51)$, we observed that independent of age, visceral AT deposition is significantly higher in men than women for a given waist circumference, and the difference was magnified with increasing waist circumference. Pouliot et al. (51) report that younger men and women have similar amounts of visceral AT for a given waist circumference. In older men and women, Schreiner et al. (41) report a curvilinear relationship between waist circumference and visceral AT, wherein men generally have more visceral AT than women, although the difference is attenuated with increasing waist circumference. The discrepant findings are not readily explained but may be due in part to differences in the landmark employed for the waist circumference measurement, as waist circumference was determined at the last rib in this study, at the waist narrowing in the study by Pouliot et al. (51) and at the umbilicus in the 
study by Schreiner (41). Wang et al. (197) report that although the difference between waist circumference measurement sites is relatively small in men $(\sim 1 \mathrm{~cm})$, in women a difference of approximately $5 \mathrm{~cm}$ is observed between the smallest (minimal waist) and largest (iliac crest) measurement sites. Thus, differences in waist circumference measurement site may have influenced the gender differences in the pattern of visceral AT deposition observed. Nevertheless, it is clear that the influence of gender on the relationship between visceral $\mathrm{AT}$ and waist circumference requires further investigation.

That menopausal status influences the relationship between waist circumference and visceral AT distribution in women beyond the effect of age per se is noteworthy. In other words, for a given waist circumference postmenopausal women have more visceral AT than a premenopausal women even after controlling age. This is in contrast to a study by Han et al. (198) that failed to demonstrate a difference in visceral AT deposition for a given waist circumference in a small cohort of women above $(n=19)$ and below $(n$ =15) 50 years of age. Nevertheless, several other studies have also demonstrated a preferential accumulation in visceral AT in postmenopausal women $(50,192,199)$. It is suggested that these body compositional changes are in part due to alterations in sex hormone levels that are associated with menopause (200-202). Indeed, ovarian hormone deficient women have a significantly higher rate of fatty acid uptake in the visceral AT depot as compared to premenopausal woman (202). Further, it has been reported that hormone replacement therapy attenuates the propensity to increase visceral AT (201). Together, these findings suggest that menopausal status should be considered when developing waist circumference cut-points for abdominal obesity related metabolic risk. 
It is known that waist circumference is a strong predictor of metabolic risk independent of BMI (5). Current evidence suggests that a waist circumference greater than $102 \mathrm{~cm}$ in men and $88 \mathrm{~cm}$ in women is associated with increased health risk (203205). It is also demonstrated that waist circumference is a strong independent predictor of visceral adiposity (29), which conveys a significant health risk $(9,206)$. Current health-related, waist circumference cut-points do not consider the influence of age on the assessment of metabolic risk (205). Indeed, this study clearly demonstrates that the amount of visceral AT associated with a given waist circumference is markedly affected by the age and gender of the individual. For example, an older man ( $>50$ years of age) with a waist circumference of $102 \mathrm{~cm}$ would be expected to have $70 \%$ more visceral AT than a 25 year old man with the same waist circumference (Table 2). Thus it is clear that differences in visceral and abdominal subcutaneous AT distribution may not be reflected by differences in its' surrogate measure waist circumference $(50,198)$. Whether the prediction of metabolic risk within each gender by waist circumference is improved by establishing age specific cut-points is unknown.

Limitations of this study warrant mention. First, our findings are restricted to Caucasian men and women. As racial differences in body fat distribution are clearly established (207), it is unclear whether the pattern or magnitude of gender and age related differences in abdominal AT deposition for a given waist circumference would be similar in other races. Second, this study did not control for differences in cardiorespiratory fitness. It has been shown that for a given waist circumference, fit men have lower levels of visceral adiposity than unfit men (46). As this was a cross-sectional study we cannot make causal or temporal inferences about the pattern of abdominal subcutaneous or 
visceral AT deposition for a given increase in waist circumference. It is also unclear whether our results were influenced by a selection bias, as individuals who continued to increase abdominal adiposity throughout their lifespan may have developed disease and would have been excluded from participation in this study.

In summary, our findings demonstrate that for a given waist circumference, abdominal AT distribution differs between genders. We confirm that for a given waist circumference, women tend to have more abdominal subcutaneous AT than men independent of age. Contrary to previous observations, men had more visceral AT than women for a given waist circumference. Further we demonstrate that visceral AT accumulation for a given waist circumference is also influenced by age and menopausal status. These findings suggest that AT distribution for a given waist circumference is altered substantially by age and gender. Whether age related changes in visceral AT influence the interpretation of health risk for a given waist circumference is unknown. 


\section{ACKNOWLEDGEMENTS}

Kuk and Lee performed the data analysis. Kuk, Lee, and Ross wrote the manuscript. Heymsfield aided in the interpretation and presentation of the results. None of the authors declared any conflicts of interest. 


\section{Table 1: Subject Characteristics}

\begin{tabular}{lcccc}
\hline \hline & Younger Men & Older Men & Younger Women & Older Women \\
\cline { 2 - 5 }$n$ & 147 & 83 & 171 & 80 \\
\hline Age & $36.2 \pm 8.0^{\mathrm{a}}$ & $60.5 \pm 8.7^{\mathrm{b}}$ & $37.6 \pm 8.6^{\mathrm{a}}$ & $66.7 \pm 8.0^{\mathrm{c}}$ \\
Body Mass Index $\left(\mathrm{kg} / \mathrm{m}^{2}\right)$ & $28.7 \pm 4.5$ & $29.3 \pm 3.8$ & $29.2 \pm 5.6$ & $27.5 \pm 4.7$ \\
Waist Circumference (cm) & $97.9 \pm 13.4^{\mathrm{a}}$ & $104.4 \pm 11.1^{\mathrm{b}}$ & $89.6 \pm 14.4^{\mathrm{c}}$ & $88.5 \pm 13.0^{\mathrm{c}}$ \\
Body Fat (\%) & $25.9 \pm 7.8^{\mathrm{a}}$ & $29.4 \pm 6.3^{\mathrm{b}}$ & $39.1 \pm 9.5^{\mathrm{c}}$ & $38.9 \pm 8.1^{\mathrm{c}}$ \\
AT Mass (kg) & & & & \\
Visceral AT & $2.34 \pm 1.44^{\mathrm{a}}$ & $3.92 \pm 1.84^{\mathrm{b}}$ & $1.49 \pm 1.01^{\mathrm{c}}$ & $1.71 \pm 1.11^{\mathrm{c}}$ \\
Abdominal Subcutaneous AT & $3.57 \pm 1.79^{\mathrm{a}}$ & $3.61 \pm 1.28^{\mathrm{a}}$ & $4.38 \pm 2.29^{\mathrm{b}}$ & $4.14 \pm 1.78^{\mathrm{ab}}$ \\
AT Area at L4-L5 (cm $\left.\mathbf{c}^{2}\right)$ & & & & \\
Visceral AT & $115.1 \pm 66.1^{\mathrm{a}}$ & $193.3 \pm 88.1^{\mathrm{b}}$ & $86.4 \pm 56.4^{\mathrm{c}}$ & $102.3 \pm 63.5^{\mathrm{ac}}$ \\
Abdominal Subcutaneous AT & $239.0 \pm 115.3^{\mathrm{a}}$ & $244.8 \pm 86.8^{\mathrm{ab}}$ & $301.9 \pm 153.8^{\mathrm{c}}$ & $288.6 \pm 124.6^{\mathrm{bc}}$ \\
\hline \hline
\end{tabular}

$\mathrm{AT}=$ Adipose Tissue

Values are mean $\pm \mathrm{SD}$.

For each variable a one-way ANOVA with Bonferroni adjustments were used to determine significance group differences at $P<0.05$. Different letters denote significance. 
Table 2: Predicted Visceral Adipose Tissue from Waist Circumference (WC) Cut-offs Associated with Increased Health Risk

\begin{tabular}{|c|c|c|c|c|c|c|}
\hline \multirow[b]{3}{*}{$\overline{W C ~(c m) ~}$} & \multicolumn{6}{|c|}{ Predicted Visceral Adipose Tissue } \\
\hline & \multicolumn{2}{|c|}{25 year old } & \multicolumn{2}{|c|}{40 year old } & \multicolumn{2}{|c|}{ Unstandardized } \\
\hline & Younger Men & Younger Women & Younger Men & Younger Women & Older Men & Older Women \\
\hline \multirow[t]{2}{*}{102} & $2.10 \mathrm{~kg}(2.09-2.11)$ & $1.50 \mathrm{~kg}(1.49-1.52)$ & $2.80 \mathrm{~kg}(2.76-2.85)$ & $2.21 \mathrm{~kg}(2.19-2.22)$ & $3.65 \mathrm{~kg}(3.60-3.71)$ & $3.08 \mathrm{~kg}(3.00-3.16)$ \\
\hline & $105.0 \mathrm{~cm}^{2}(104.7-105.3)$ & $95.3 \mathrm{~cm}^{2}(95.0-95.5)$ & $135.4 \mathrm{~cm}^{2}(133.3-137.4)$ & $125.6 \mathrm{~cm}^{2}(124.1-127.1)$ & $179.4 \mathrm{~cm}^{2}(176.1-182.7)$ & $167.9 \mathrm{~cm}^{2}(163.6-172.1)$ \\
\hline \multirow[t]{2}{*}{94} & $1.59 \mathrm{~kg}(1.57-1.60)$ & $1.22 \mathrm{~kg}(1.18-1.25)$ & $2.11 \mathrm{~kg}(2.10-2.12)$ & $1.74 \mathrm{~kg}(1.73-1.75)$ & $2.49 \mathrm{~kg}(2.47-2.51)$ & $2.35 \mathrm{~kg}(2.31-2.39)$ \\
\hline & $81.6 \mathrm{~cm}^{2}(80.6-82.7)$ & $76.4 \mathrm{~cm}^{2}(75.0-77.7)$ & $105.8 \mathrm{~cm}^{2}(105.5-106.2)$ & $100.6 \mathrm{~cm}^{2}(100.5-100.6)$ & $127.6 \mathrm{~cm}^{2}(126.9-128.3)$ & $131.3 \mathrm{~cm}^{2}(129.3-133.3)$ \\
\hline \multirow[t]{2}{*}{88} & $1.21 \mathrm{~kg}(1.17-1.24)$ & $1.00 \mathrm{~kg}(0.96-1.05)$ & $1.59 \mathrm{~kg}(1.57-1.60)$ & $1.39 \mathrm{~kg}(1.36-1.41)$ & $1.62 \mathrm{~kg}(1.55-1.70)$ & $1.80 \mathrm{~kg}(1.80-1.81)$ \\
\hline & $64.1 \mathrm{~cm}^{2}(62.0-66.2)$ & $62.2 \mathrm{~cm}^{2}(60.0-64.4)$ & $83.7 \mathrm{~cm}^{2}(82.7-84.6)$ & $81.8 \mathrm{~cm}^{2}(80.7-82.8)$ & $88.7 \mathrm{~cm}^{2}(85.0-92.4)$ & $103.8 \mathrm{~cm}^{2}(103.6-104.1)$ \\
\hline \multirow[t]{2}{*}{80} & $0.69 \mathrm{~kg}(0.63-0.75)$ & $0.72 \mathrm{~kg}(0.66-0.78)$ & $0.89 \mathrm{~kg}(0.84-0.94)$ & $0.92 \mathrm{~kg}(0.87-0.96)$ & $0.47 \mathrm{~kg}(0.31-0.62)$ & $1.07 \mathrm{~kg}(1.03-1.11)$ \\
\hline & $40.7 \mathrm{~cm}^{2}(37.3-44.2)$ & $43.3 \mathrm{~cm}^{2}(40.0-46.6)$ & $54.1 \mathrm{~cm}^{2}(51.5-56.8)$ & $56.7 \mathrm{~cm}^{2}(54.2-59.2)$ & $36.8 \mathrm{~cm}^{2}(29.2-44.5)$ & $67.2 \mathrm{~cm}^{2}(65.2-69.2)$ \\
\hline
\end{tabular}

The values are the predicted visceral adipose tissue $(95 \% \mathrm{CI})$ derived from the regression equations reported in Figure 3. 
Figure 1 - Gender Differences in the Association between Waist Circumference (WC) and Total Abdominal Adipose Tissue (TAAT).

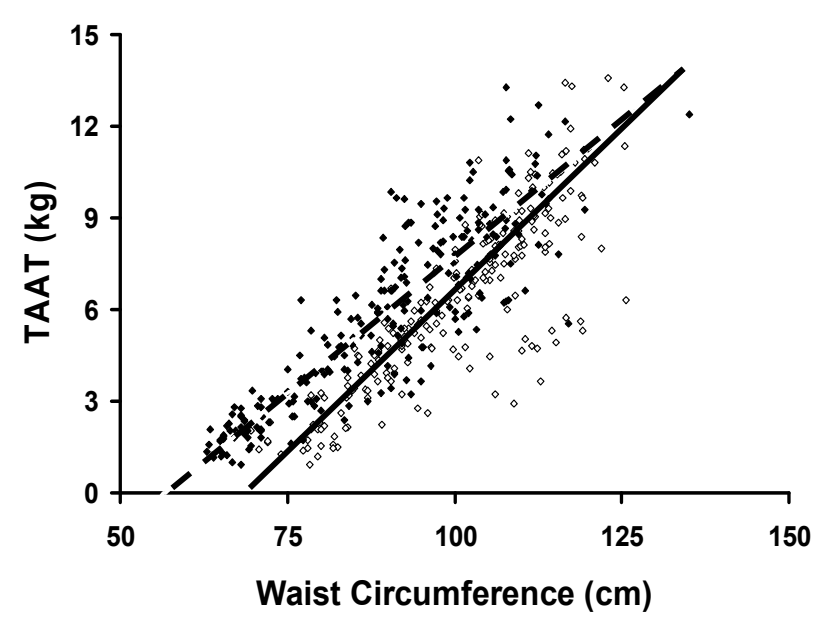

The 3-factor interaction was not significant $(\mathrm{P}>0.05)$. There was a significant gender*WC $(\mathrm{P}<0.05)$, but not age*WC interaction $(\mathrm{P}>0.05)$. The regression line for men $(n=230)$ is the solid line and the dotted line for women $(n=251)$. Values for men are in open diamonds $(\diamond)$ and values for women are in closed diamonds $(\diamond)$. TAAT Men $_{\text {. }}$ $(\mathrm{kg})=-14.5+0.21(\mathrm{WC})$, TAAT $_{\text {Women }}(\mathrm{kg})=-10.1+0.18(\mathrm{WC})$. 
Figure 2 - Gender Differences in the Association between Waist Circumference (WC) and Abdominal Subcutaneous Adipose Tissue (ASAT).

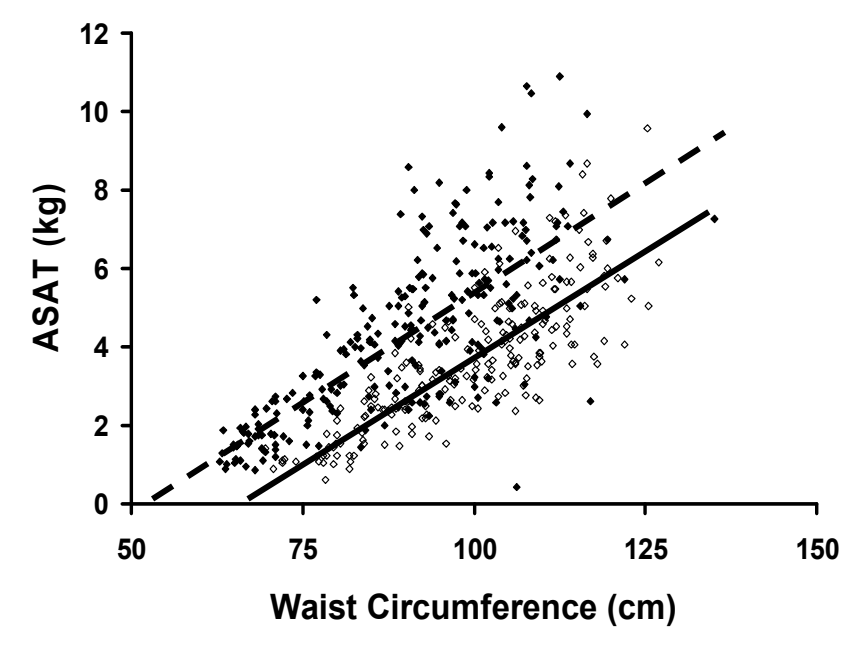

The 3-factor and 2-factor interactions were not significant $(\mathrm{P}>0.05)$. There was a significant main effect of gender $(\mathrm{P}<0.05)$. The regression line for men $(n=230)$ is in the solid line and the dotted line for women $(n=251)$. Values for men are in open diamonds $(\diamond)$ and values for women are in closed diamonds $(\diamond) . \operatorname{ASAT}_{\text {Men }}(\mathrm{kg})=-7.57$ $+0.11(\mathrm{WC}), \mathrm{ASAT}_{\text {Women }}(\mathrm{kg})=-5.74+0.11(\mathrm{WC})$. 
Figure 3 - Gender Differences in the Association between Waist Circumference (WC) and Visceral Adipose Tissue (VAT).
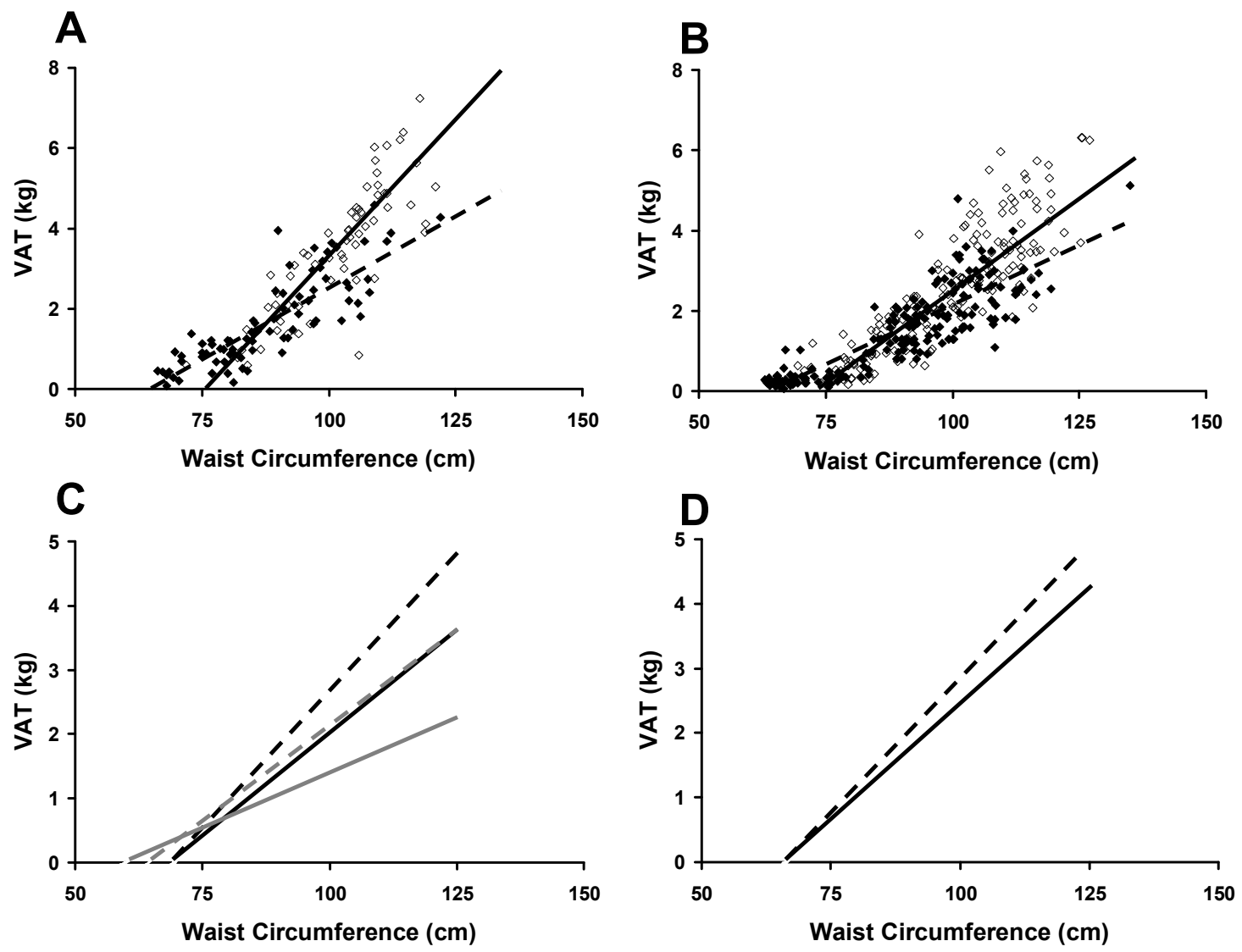

There was a significant 3 -factor interaction (age*gender*WC, P <0.05). Panel A:

Relationship between waist circumference and visceral AT mass in 83 older men (solid line), and 80 older women (dashed line). $\operatorname{VAT}_{\text {Older Men }}(\mathrm{kg})=-11.12+0.145(\mathrm{WC})$, $\operatorname{VAT}_{\text {Older Women }}(\mathrm{kg})=-6.25+0.091(\mathrm{WC})$. Panel B: Relationship between waist circumference and visceral AT mass in 147 younger men (solid line), and 171 younger women (dashed line). Values for men are in open diamonds $(\diamond)$, values for women are in closed diamonds $(\diamond)$. $\operatorname{VAT}_{\text {Younger Men }}(\mathrm{kg})=-6.59+0.092(\mathrm{WC}), \mathrm{VAT}_{\text {Younger Women }}(\mathrm{kg})$ $=-3.74+0.058(\mathrm{WC})$. Panel C: Relationship between waist circumference and visceral AT mass standardized to 25- (solid line) and 40- (dotted line) years of age in 147 younger 
men (black lines) and 171 women women (grey lines). $\operatorname{VAT}_{\text {Men }}(\mathrm{kg})=-1.69+$ $0.026(\mathrm{WC})-0.109($ Age $)+0.0015\left(\right.$ Age $\left.^{*} \mathrm{WC}\right), \mathrm{VAT}_{\text {Women }}(\mathrm{kg})=0.58-0.0024(\mathrm{WC})-$ 0.109(Age) + 0.0015(Age*WC). Panel D: Relationship between waist circumference and visceral AT in premenopausal and postmenopausal women standardized to 50 years of age. There was a significant menopausal status*WC interaction $(\mathrm{P}<0.05)$. Data for the premenopausal women is in the solid line $(n=171)$, and postmenopausal women in the dashed line $(n=80) . \operatorname{VAT}_{\text {Pre Women }}(\mathrm{kg})=-0.14+0.0045(\mathrm{WC})-0.099($ Age $)+$ $0.0014\left(\right.$ Age $\left.^{*} \mathrm{WC}\right), \mathrm{VAT}_{\text {Post Women }}(\mathrm{kg})=-8.01+0.115(\mathrm{WC})+0.050($ Age $)+$ 0.0006(Age*WC). 


\section{Chapter 4. Manuscript 2}

This manuscript has been published in Diabetes Care. The co-authors of this manuscript are Steven N. Blair, Timothy S. Church and Robert Ross. This research was supported in part by research grants from the Canadian Institutes of Health Research to Robert Ross (MT13448) and the National Institutes of Health to Steven N. Blair and Timothy S. Church (AG-06945). 
Does Measurement Site for Visceral and Abdominal Subcutaneous Adipose Tissue Alter Associations with the Metabolic Syndrome? 


\begin{abstract}
Objective: To determine whether the associations between visceral (VAT), abdominal subcutaneous adipose tissue (ASAT) and the metabolic syndrome (MetS) are altered depending on measurement site for VAT and ASAT, and the definition used to identify MetS.
\end{abstract}

Research Methods and Procedures: Total VAT and ASAT volume was derived using approximately 37 contiguous CT images from T10-T11 to L5-S1 in 85 men. CT images obtained at 8 intervertebral locations (e.g., L4-L5, L3-L4, etc) were used to determine associations between partial volumes (single images) and MetS. MetS was defined using the National Cholesterol Education Program (NCEP) and International Diabetes Federation (IDF) criteria. Logistic regression was used to calculate the odds ratio (OR, per SD increase in AT).

Results: For total and all partial volumes, VAT was more strongly associated with MetS than ASAT independent of MetS criteria. The OR for NCEP MetS was higher for total VAT volume $(\mathrm{OR}=7.26)$ and for the partial volumes at T12-L1 $(\mathrm{OR}=7.46)$ and L1-L2 $(\mathrm{OR}=8.77)$ than at the L4-L5 level $(\mathrm{OR}=3.94)$. The OR for MetS was not substantially different among the ASAT measures (OR 2.6). A similar pattern of association was observed using the IDF MetS criteria.

Discussion: Measurement site for VAT, but not ASAT, has a substantial influence on the magnitude of the association with both MetS definitions. However, because VAT remained significantly associated with MetS regardless of measurement site, the clinical interpretation was unaltered by measurement protocol or MetS definition. 


\section{INTRODUCTION}

Mounting evidence suggests that the metabolic syndrome (MetS), generally characterized using the National Cholesterol Education Program (NCEP) criteria, is a powerful predictor of morbidity and mortality (208). Because abdominal obesity is central to the NCEP MetS definition, studies have investigated the relationship between visceral and abdominal subcutaneous adipose tissue (VAT and ASAT) with MetS (36, 38). Carr et al. (36) and Goodpaster et al. (38) report that VAT is associated with increased prevalence of MetS. Conversely, ASAT is not consistently reported to be a significant correlate of MetS (36), or its individual components (38). This may be due in part to variation in the measurement site selected for quantifying abdominal AT. Although L4-L5 is the most commonly used landmark for measuring VAT and ASAT, there is reason to believe that L4-L5 is not the ideal site for quantifying abdominal AT or predicting obesity related metabolic risk. Recent studies report that a single image in the upper abdomen (i.e. L1-L2 or L2-L3) is a more suitable surrogate for total VAT $(89,91$, 92), and ASAT volume (89) than L4-L5. If one assumes that the ability to predict the total VAT or ASAT volume will translate to a corresponding ability to predict MetS, then VAT and ASAT obtained at L1-L2 or L2-L3 may be better predictors of MetS than L4L5. Further, there is evidence to suggest that a greater deposition of the more metabolically active visceral adipocytes within the omental and mesenteric depots are located within the region of L1-L2 to L3-L4 $(58,209)$. Taken together these observations suggest that VAT measures in the upper abdomen should have stronger associations with MetS than measures in the lower abdomen. No study has systematically determined the measurement site for VAT and ASAT that is optimal for 
determination of MetS. Further, although several criteria exist for identifying MetS (210), the NCEP and International Diabetes Federation (IDF) criteria are the most readily employed in clinical settings. Unknown is whether the criteria used to identify MetS alters the association with VAT and ASAT.

The primary purpose of this study was, therefore, to determine whether the associations between VAT, ASAT and MetS were altered depending on the site of measurement for VAT and ASAT and/or the criteria used to identify MetS. 


\section{RESEARCH DESIGN AND METHODS}

\section{Subjects}

Subjects consisted of a subset of 85 Caucasian men selected from a cohort of men who had received an abdominal CT scan as part of a preventive medicine diagnostic examination at the Cooper Clinic in Dallas, Texas. The details of this examination are described elsewhere (37). All subjects gave their informed written consent prior to participation in the examination, and the study was reviewed and approved annually by The Cooper Institute Institutional Review Board.

\section{Biochemistry Analyses}

Venous blood samples were taken from the antecubital vein in the morning following an overnight fast of at least 12 hours, and were analyzed using automated methods in a laboratory that participates in and meets quality control standards of the Centers for Disease Control and Prevention Lipid Standardization Program. Measures included total serum triglycerides, high-density lipoprotein (HDL) cholesterol and fasting glucose.

MetS was defined according to the criteria established by NCEP, Adult Treatment Panel III (211) and IDF (212). Participants were classified as having NCEP MetS if they had 3 or more of the following 5 risk factors: (1) high systolic blood pressure $\geq 130 \mathrm{~mm}$ $\mathrm{Hg}$, or the use of antihypertensive medication; (2) abdominal obesity (waist circumference $>102 \mathrm{~cm}$ ); (3) high triglyceride level ( $\geq 150 \mathrm{mg} / \mathrm{mL})$; (4) low HDL cholesterol level (<40 mg/mL); and (5) high fasting plasma glucose level $(\geq 110 \mathrm{mg} / \mathrm{mL})$. 
Participants were classified as having IDF MetS if they had central obesity (waist circumference $\geq 94 \mathrm{~cm}$ ) and 2 or more of the following 4 risk factors: (1) high systolic blood pressure $\geq 130 \mathrm{~mm} \mathrm{Hg}$, or the use of antihypertensive medication; (2) high triglyceride level ( $\geq 150 \mathrm{mg} / \mathrm{mL})$; (3) low HDL cholesterol level $(<40 \mathrm{mg} / \mathrm{mL})$; and (4) high fasting plasma glucose level ( $\geq 100 \mathrm{mg} / \mathrm{mL})$.

\section{Measurement of abdominal AT by CT}

Axial images of the abdominal region were obtained in an electron beam CT scanner (Imatron, General Electric, Milwaukee, WI) using a standard protocol (213). Subjects were examined in a supine position with their arms extended above their heads. Approximately 40 contiguous transverse images ( $6 \mathrm{~mm}$ thickness, $0 \mathrm{~mm}$ inter-space) were acquired from the mid-region of the iliac crest to the caudal region of the heart. Images were obtained using $130 \mathrm{kV}$ and $630 \mathrm{~mA}$ with a $480 \mathrm{~mm}$ field of view and a 512 x 512 matrix, resulting in a pixel size of $0.7813 \mathrm{~mm}^{2}$. The CT data obtained in Dallas were transferred electronically to Queen's University in Kingston for analysis using specially designed image analysis software (Tomovision, Inc., Montreal, Canada) as previously described (37).

The inter-observer reliability error for VAT and ASAT measurement for two observers' analyses of the same L4-L5 image $(\mathrm{n}=40)$ was $\sim 3 \%$ and $\sim 1 \%$, respectively (214). 


\section{Determination and calculation of abdominal AT partial and total volumes}

For each subject a single axial image that most closely approximated the center of the inter-vertebral space or vertebral body was identified for L4-L5, L3-L4, etc. using known anatomical landmarks (215). The partial volumes $\left(\mathrm{cm}^{3}\right)$ of AT were calculated as

the AT tissue areas $\left(\mathrm{cm}^{2}\right)$ at the eight inter-vertebral spaces within the region of T10-T11 to L5-S1 multiplied by the slice thickness $(6 \mathrm{~mm})$. Total VAT and ASAT volume was determined from approximately 37 contiguous images from T10-T11 to L5-S1 (criterion method). The total volume $\left(\mathrm{cm}^{3}\right)$ of AT was calculated as the sum of all partial AT tissue volumes $\left(\mathrm{cm}^{3}\right)$ within the region of T10-T11 to L5-S1.

\section{Statistical analyses}

Group data are presented as means \pm SD. Pearson's correlations and linear regression analyses were performed to determine the relationship between the criterion measure of abdominal AT volume, and the regional AT areas. The strength of the correlations was compared by using the Hotelling method (216). The strength of the standard error of estimate values was compared using Pitman's test (217). Logistic regression was used to calculate the odds-ratios for MetS. The odds-ratios were expressed per standard deviation increase to facilitate comparisons between AT variables. To examine these associations in overweight or obese men who are at increased risk for metabolic disorders, we analyzed data from men with a BMI $\geq 27 \mathrm{~kg} / \mathrm{m}^{2}(\mathrm{n}=40)$. All statistical procedures were performed using SAS v8. 


\section{RESULTS}

The subject characteristics are listed in Table 1. The pattern of VAT and ASAT distribution within the abdomen is shown in Figure 1. The relationships between each of the partial volumes obtained at the inter-vertebral spaces between T10-T11 to L5-S1 with the total VAT and ASAT volume are provided in Table 2. As expected, all the VAT and ASAT partial volumes derived from single images were significantly correlated with the respective total volumes for VAT $\left(R^{2}=0.65\right.$ to 0.96$)$ and $\operatorname{ASAT}\left(R^{2}=0.80\right.$ to 0.96$)$ respectively (Table 2). For VAT, the partial volumes at the L1-L2 and L2-L3 intervertebral spaces had significantly higher correlation coefficients with total volume and lower SEEs than all the other images (Table 2, $P<0.05$ ). For subcutaneous AT, the partial volume at the L2-L3 inter-vertebral space had a significantly higher correlation coefficient with total volume and lower SEE than all the other images $(P<0.05)$ with the exception of the image obtained at the level of L1-L2 (Table 2, $P>0.10$ ).

The MetS was present in $16.5 \%$ and $28.2 \%$ of the sample as defined by NCEP and IDF respectively. The association between VAT and ASAT partial and total volumes with the NCEP and IDF MetS is shown in Figure 2. Independent of measurement site or MetS definition, the ORs between VAT and MetS were higher than for ASAT. The OR for the NCEP MetS was higher for total VAT volume $(\mathrm{OR}=7.26)$ and for the partial volumes at T12-L1 $(\mathrm{OR}=7.46)$ and L1-L2 $(\mathrm{OR}=8.77)$ than for L4-L5 $(\mathrm{OR}=3.94)$. Similarly, the OR for the IDF MetS was higher for total VAT volume $(\mathrm{OR}=4.95)$ and for the partial volumes at T12-L1 $(\mathrm{OR}=7.08)$ and L1-L2 $(\mathrm{OR}=5.54)$ than for L4-L5 $(\mathrm{OR}=2.99)$. The association between the NCEP and IDF MetS, and ASAT was not altered by measurement site (ORs $\sim 2.6$, Figure 2). 
For both VAT and ASAT the observations were similar in a subsample of men with a BMI $\geq 27 \mathrm{~kg} / \mathrm{m}^{2}$, although the magnitude of the associations was diminished using either MetS definition (Figure 2). Indeed, VAT was significantly associated with NCEP MetS at all measurement sites, and was significantly associated with IDF MetS at intervertebral spaces in the upper abdomen within the region of T10-T11 to L2-L3 (P $\leq 0.05$ ). For those with a BMI $>27 \mathrm{~kg} / \mathrm{m}^{2}$, ASAT was not significantly associated with either MetS definition regardless of measurement site $(\mathrm{P}>0.10)$. 


\section{DISCUSSION}

The primary finding of this study was that the measurement site for VAT has a substantial influence on the magnitude of the odds-ratios obtained for MetS. Indeed, VAT at the T12-L1 and L1-L2 landmarks had much stronger associations with MetS than corresponding measures at L4-L5. However, regardless of measurement site and MetS criteria, VAT was significantly associated with MetS and thus, the clinical interpretation was unaltered by measurement protocol.

In this study, regardless of measurement site and criteria used to identify MetS, VAT was more strongly associated with MetS than ASAT. These findings extend prior observations that report VAT is a stronger predictor of prevalent NCEP MetS than ASAT $(36,38)$. Carr et al. (36), measured VAT and ASAT at the umbilicus, which is proximal to measures at L4-L5. Accordingly, the odds ratios reported by Carr et al. (36) for VAT $(\mathrm{OR}=3.8)$ and ASAT $(\mathrm{OR}=2.9)$ were similar to those reported in this study at L4-L5 (VAT: OR =3.9, ASAT: OR =2.8). However, the OR we report for MetS when VAT was measured at L4-L5 was substantially lower than those observed in the upper abdomen. For example, measurement of VAT at L1-L2 resulted in a two-fold higher OR than L4-L5 for NCEP (OR = 8.8 vs. 3.9) and IDF ( $\mathrm{OR}=5.5$ vs. 2.9) MetS. Conversely, the association between ASAT for either definition of MetS was similar regardless of measurement site $(\mathrm{OR}=2.1$ to 3.0$)$.

It is unclear why measurement site would influence the strength of the association between VAT and MetS, but not ASAT and MetS. One reason may be that, whereas the relationship between a single image (e.g. ASAT at a given level) and the total ASAT volume is generally similar throughout the abdomen, we observed marked differences in 
the association between VAT at a single level and the total volume of VAT. If one assumes that the ability to predict VAT or ASAT volume will translate to a corresponding ability to predict MetS, then a single image at L1-L2 or L2-L3 may be a better predictor of MetS than L4-L5 simply because it is a better predictor of the total AT volume. Indeed, a single measure at L4-L5 accounted for significantly less variance and had two-fold higher error estimates than measures at L1-L2 or L2-L3 $\left(\mathrm{R}^{2}=0.80\right.$ vs. 0.96 and $\mathrm{SEE}=22 \%$ vs. $10 \%$, respectively). Conversely, the differences in the variance explained and the error estimates for ASAT measured at L4-L5 versus L1-L2 or L2-L3 were subtle $\left(\mathrm{R}^{2}=0.92\right.$ vs. 0.95 and $\mathrm{SEE}=10.5 \%$ vs. $7.5 \%$, respectively). As such, measurement site may be expected to have a greater influence on VAT associated metabolic risk than ASAT.

It is also plausible that differences in the metabolic activity of adipocytes within the VAT and ASAT depots contribute to differences in predicting MetS. Visceral adipocytes in the omental and mesenteric depots are portally drained, and are more sensitive to catecholamine-stimulated lipolysis by comparison to the non-portally drained retroperitoneal adipocytes (209). Similarly, it is reported that changes in adiponectin levels are largely accounted for by changes in the secretion from the omental adipocytes (58). Although the mechanistic links between VAT and metabolic risk factors remains to be firmly established, it is reported that the greatest deposition of omental and mesenteric fat is located in the upper abdomen within the region between L1-L2 and L3-L4 (89), and thus, VAT in the upper abdomen may be expected to have a greater impact on hepatic metabolism and metabolic derangements than the lower abdomen. Whether there are 
similar regional differences in ASAT metabolism in the upper and lower abdomen is unknown.

We also examined whether the criteria used to identify MetS would influence the association with VAT and ASAT. The most clinically relevant MetS criteria were proposed by NCEP in 2001 (211). The recently proposed IDF consensus criteria for identification of MetS (212) builds upon the NCEP criteria, but differs in two key aspects. First, the IDF lowered the threshold for waist circumference $(102 \mathrm{~cm}$ to $94 \mathrm{~cm})$ and fasting glucose ( 6.1 to $5.6 \mathrm{mmol} / \mathrm{L})$. Second, waist circumference is a required component of MetS under the IDF criteria, rather than an optional component as employed with NCEP. Given the lower values for waist circumference and glucose it is not surprising that the IDF MetS classifies a larger population with MetS than NCEP ( $16.5 \%$ vs. $28.2 \%$, respectively). Nevertheless, we demonstrate a similar influence of measurement site wherein VAT measures in the upper abdomen are more strongly associated with IDF MetS. However, the odds ratios for IDF MetS for VAT were generally lower than those associated with NCEP MetS. This may be partially explained by the observation that the additional individuals captured using the IDF MetS criteria have a lower waist circumference and hence, lower VAT. As a result, the difference in VAT between individuals with or without MetS (NCEP: $74 \mathrm{~cm}^{2}$, IDF: $59 \mathrm{~cm}^{2}$ at L4-L5) is reduced using the IDF guidelines, consequently weakening the association between VAT and IDF MetS as compared to the NCEP criteria. Nevertheless, neither MetS criteria nor measurement site altered the statistical significance of the association between VAT or ASAT and MetS, and thus the clinical observation was unaltered. 
The strengths and limitations of this study include the use of a relatively small opportunistic sample of men who had acquired abdominal CT images at the Cooper Institute. The men in this study are predominantly white, and from a middle-to-upper class population. This limits the generalizability of the results of our study, but should not affect the internal validity. Indeed, the homogeneity of our study group on socioeconomic factors is a benefit because it reduces the likelihood of confounding by these factors. Future studies should examine these observations hold true in women and other racial populations as there are clear gender and racial differences in fat distribution (207) and adipocyte metabolism in vitro (218). Further, studies should investigate whether this pattern of association remains true with abdominal AT changes over time, or whether the measurement site influences the relationship between changes in abdominal AT and obesity related metabolic risk factors to determine the optimal landmark for the quantification of abdominal adiposity.

In summary, this study is the first to provide evidence that VAT has a stronger association with MetS than ASAT independent of measurement site and MetS criteria. Although the strength of the association was affected by the measurement site, VAT remained significantly associated with MetS regardless of measurement methodology. Thus, the clinical observation and conclusion remains the same regardless of measurement site. This reinforces the notion that elevated visceral adiposity is associated with increased risk of incident MetS as characterized by NCEP and IDF, and that VAT should be a primary target for pharmacological and lifestyle based intervention. 


\section{ACKNOWLEDGEMENTS}

The authors would like to extend a special thanks to Elisa Priest and Michael LaMonte for their work related to the management of the CT data. This research was supported in part by research grants from the National Institutes of Health to Steven N. Blair (AG06945), and from the Canadian Institutes of Health Research to Robert Ross (MT13448). 
Table 1. Subject Characteristics

\begin{tabular}{|c|c|c|}
\hline $\mathrm{n}=85$ & Mean \pm SD & Range \\
\hline Age (y) & $51.0 \pm 8.0$ & $30-67$ \\
\hline $\operatorname{BMI}\left(\mathrm{kg} / \mathrm{m}^{2}\right)$ & $27.0 \pm 3.6$ & $21.2-35.8$ \\
\hline Waist Circumference (cm) & $95.2 \pm 10.4$ & $74-122$ \\
\hline VAT Volume (L) & $3.1 \pm 1.5$ & $0.3-6.4$ \\
\hline ASAT Volume (L) & $3.7 \pm 1.3$ & $1.3-7.9$ \\
\hline VAT Area L4-L5 (cm²) & $139.0 \pm 60.2$ & $20.7-272.9$ \\
\hline ASAT Area L4-L5 $\left(\mathrm{cm}^{2}\right)$ & $223.3 \pm 77.4$ & $77.7-416.1$ \\
\hline Fasting Glucose (mg/dL) & $101.5 \pm 9.2$ & $83-133$ \\
\hline Fasting Triglycerides (mg/dL) & $131.0 \pm 54.6$ & $50-295$ \\
\hline HDL Cholesterol (mg/dL) & $46.2 \pm 10.3$ & $28-73$ \\
\hline Systolic Blood Pressure $(\mathrm{mmHg}) *$ & $124.4 \pm 13.3$ & $100-176$ \\
\hline
\end{tabular}


Table 2. The relationships between the VAT and ASAT partial volumes at each inter-vertebral space with the corresponding total VAT and ASAT volume measures.

\begin{tabular}{|c|c|c|c|c|}
\hline \multirow{2}{*}{$\begin{array}{l}\text { Region of } \\
\text { Measure }\left(\mathrm{cm}^{3}\right)\end{array}$} & \multicolumn{2}{|c|}{$\begin{array}{l}\text { Total VAT Volume } \\
\text { (T10-T11 to L5-S1), L }\end{array}$} & \multicolumn{2}{|c|}{$\begin{array}{l}\text { Total ASAT Volume } \\
\text { (T10-T11 to L5-S1), L }\end{array}$} \\
\hline & $R^{2}$ & SEE, L (\%) & $R^{2}$ & SEE, L (\%) \\
\hline T10-T11 & $0.68^{\mathrm{a}}$ & $0.85(27.7)^{\mathrm{a}}$ & $0.85^{\mathrm{a}}$ & $0.52(14.2)^{\mathrm{a}}$ \\
\hline T11-T12 & $0.69^{\mathrm{a}}$ & $0.82(26.9)^{\mathrm{a}}$ & $0.89^{\mathrm{b}}$ & $0.45(12.3)^{b}$ \\
\hline T12-L1 & $0.90^{\mathrm{b}}$ & $0.47(15.5)^{b}$ & $0.94^{\mathrm{c}}$ & $0.34(9.2)^{\mathrm{c}, \mathrm{d}}$ \\
\hline L1-L2 & $0.96^{\mathrm{c}}$ & $0.29(9.6)^{\mathrm{c}}$ & $0.95^{\mathrm{d}}$ & $0.29(7.8)^{\mathrm{d}, \mathrm{e}}$ \\
\hline L2-L3 & $0.96^{\mathrm{c}}$ & $0.31(10.1)^{\mathrm{c}}$ & $0.96^{\mathrm{d}}$ & $0.26(7.2)^{\mathrm{e}}$ \\
\hline L3-L4 & $0.91^{\mathrm{b}}$ & $0.44(14.3)^{b}$ & $0.94^{\mathrm{c}}$ & $0.32(8.7)^{\mathrm{c}, \mathrm{d}}$ \\
\hline L4-L5 & $0.80^{\mathrm{d}}$ & $0.65(21.6)^{d}$ & $0.92^{\mathrm{e}}$ & $0.38(10.5)^{b, c}$ \\
\hline L5-S1 & $0.65^{\mathrm{a}}$ & $0.88(28.8)^{\mathrm{a}}$ & $0.80^{\mathrm{a}}$ & $0.59(16.1)^{\mathrm{a}}$ \\
\hline
\end{tabular}

All correlations were significant at $P<0.001$.

Different superscript letters indicate significant difference in the correlation coefficient using the Hotelling method $(P<0.05)$, and SEE using the Pitman's Test $(P<0.05)$. 
Figure 1 - VAT (A) and ASAT (B) Deposition at Various Measurement Sites across the Abdomen in 85 Men.
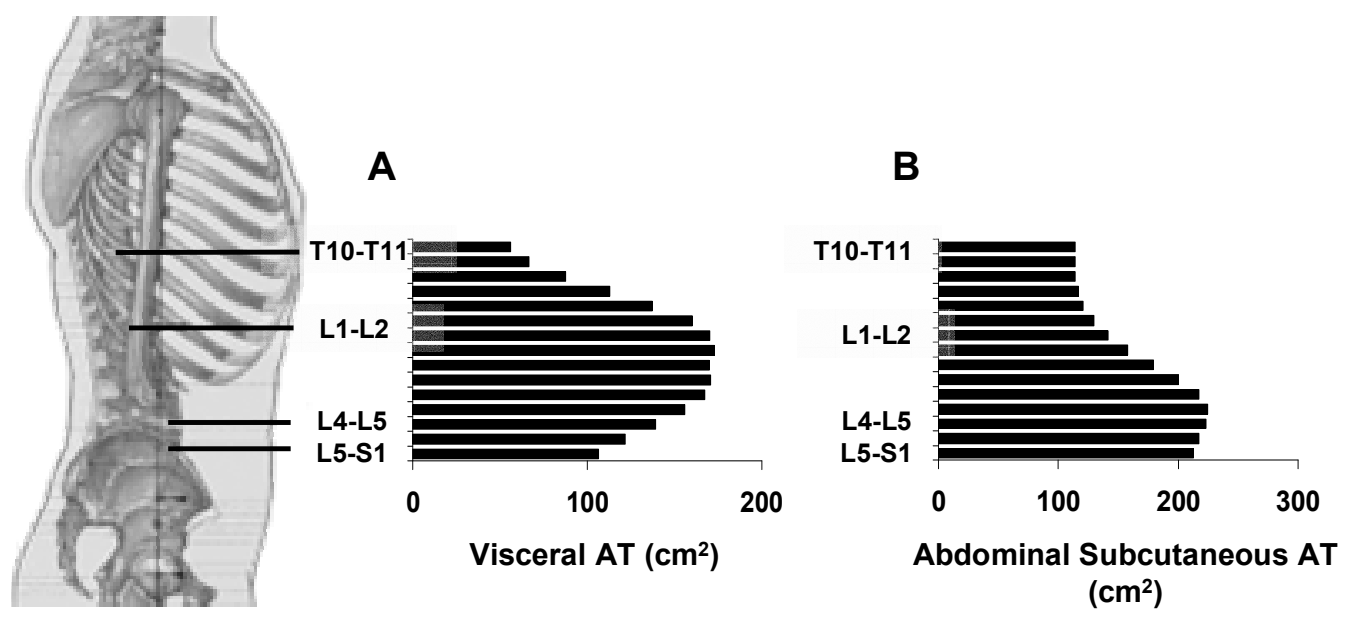
Figure 2 - Odds Ratios for Prevalent MetS using NCEP (A) and IDF (B) Criteria According to Measurement Location for VAT and ASAT in All Men and Men with a $B M I \geq 27 \mathrm{~kg} / \mathrm{m}^{2}$.
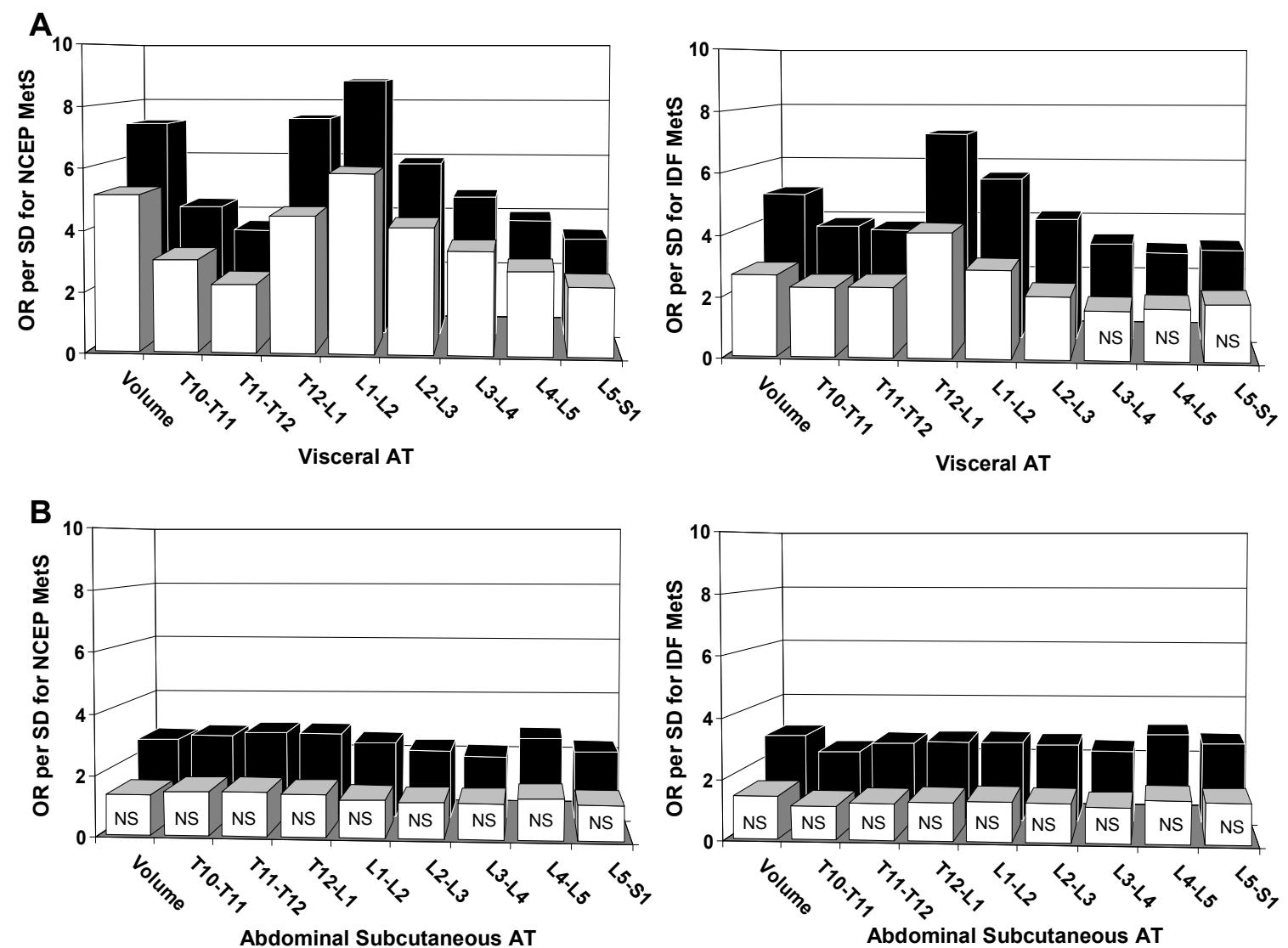

Black bars $=$ All men $(n=85)$, White Bars $=$ Men with BMI $\geq 27 \mathrm{~kg} / \mathrm{m}^{2}(\mathrm{n}=40)$.

OR for each measure significant at $\mathrm{P}<0.05, \mathrm{NS}=$ Not Significant $(\mathrm{P}>0.05),{ }^{*} \mathrm{P}=0.05$ 


\section{Chapter 5. Manuscript 3}

This manuscript has been published in Obesity. The co-authors of this manuscript are Peter T. Katzmarzyk, Milton Z. Nichaman, Steven N. Blair, Timothy S. Church and Robert Ross. This research was supported in part by research grants from the Canadian Institutes of Health Research to Robert Ross (MT13448) and the National Institutes of Health to Steven N. Blair (AG-06945) and Milton Z. Nichaman (HL-62508). 
Visceral Fat Is an Independent Predictor of All-Cause Mortality in Men 


\begin{abstract}
Objective: To examine the independent associations between abdominal fat (visceral and subcutaneous), liver fat and all-cause mortality.
\end{abstract}

Research Methods and Procedures: Participants included 291 men (97 decedents and 194 controls, mean [SD], age, 56.4 [12.0] years) who received a computed tomography (CT) examination at the preventive medicine clinic in Dallas, Texas between 1995 and 1999 , with a mean mortality follow-up of $2.2 \pm 1.3$ years. Abdominal fat was determined using contiguous CT images from the L3-L4 to L4-L5 intervertebral space. Liver fat was assessed using the CT-determined liver attenuation value, which is inversely related to liver fat. Logistic regression was used to determine the association between the independent abdominal fat depots and all-cause mortality.

Results: During the study there were 97 deaths. Visceral fat (OR per SD: 1.83 [95\% CI: $1.23-2.73])$, abdominal subcutaneous fat (1.44 [1.02-2.03]), liver fat (0.64 [0.46$0.87])$ and waist circumference (1.41 [1.01-1.98]), were all significant individual predictors of mortality after controlling for age and length of follow-up. In a model including all three fat measures (subcutaneous, visceral and liver fat), age, and length of follow-up, only visceral fat (1.93 [1.15-3.23]) was a significant predictor of mortality. Discussion: Visceral fat is a strong, independent predictor of all-cause mortality in men.

Key Words: Liver fat, abdominal fat, computed tomography 


\section{INTRODUCTION}

It is well established that abdominal obesity is associated with increased morbidity independent of age, race and gender $(20,219)$. Abdominal adiposity as measured by waist circumference is a significant predictor of morbidity (5) and mortality (8) independent of body mass index (BMI). The association between waist circumference and metabolic risk may be explained in part by its strong association with visceral adiposity (33). Visceral fat is a strong, independent predictor of dyslipidemia (9, $54,98)$ and insulin resistance $(98,220)$, and changes in visceral fat are associated with concomitant changes in glucose tolerance and insulin resistance (12). In prospective studies visceral fat predicts the development of type 2 diabetes (39). Although the association between visceral adiposity and morbidity appears to be well established, to date no study has examined the association between visceral fat and mortality using direct measures of visceral fat.

Emerging evidence suggests that fat deposition within the liver is another component of fat distribution that is independently linked to dyslipidemia (98) and insulin resistance $(93,103)$. Although the association of these fat depots with metabolic risk and morbidity $(9,52,54,93,98,103,220)$ is established, their association with mortality is unknown. Evaluation of the independent associations between abdominal (subcutaneous and visceral) and liver fat with mortality would clarify and reinforce primary targets for obesity reduction and thus, would have direct relevance to the development of public health policy and clinical recommendations. Therefore, we investigated the independent associations between visceral, abdominal subcutaneous and liver fat deposition and mortality in men. 


\section{RESEARCH DESIGN AND METHODS}

\section{Participants}

Study participants consisted of a subset of 291 men (97 decedents and 194 controls), who were selected from a larger predominantly Caucasian ( $\sim 98 \%)$ cohort who received a computed tomography (CT) examination of the abdomen region, as part of a preventive medicine diagnostic exam at the Cooper Clinic in Dallas, Texas, between 1995 and 1999, and were followed through December 31, 1999. Study participants are from mid- to upper social economic status groups ( $\sim 80 \%$ are college graduates), and typically are or were employed in executive or professional positions. The National Death Index was used to identify potential deaths and cause, and official death certificates were obtained and were cross-referenced with the participant's clinical record to confirm a match. To create a control group that was reflective of our study cohort, the control subjects were randomly selected from survivors that were alive at the time of death of the decedent. The mean length of follow-up was $2.2 \pm 1.3$ years.

All study participants gave their informed written consent prior to participation in the examination, and the study was reviewed and approved annually by The Cooper Institute Institutional Review Board. All study participants completed a questionnaire on demographic factors, health habits, medical history and a CT exam of the abdomen region. Self-reported height and weight were used to calculate body mass index.

\section{Procedures}

Axial images of the abdomen region were obtained using an electron beam computed tomography (CT, Imatron, General Electric, Milwaukee, WI) scanner with the 
subject lying in a supine position (213). Approximately 40 contiguous images (6 mm thickness) were acquired from the distal iliac crest to the caudal region of the heart. Images were obtained using standard procedures and analyzed using specialized image analysis software (Tomovision, Inc., Montreal, Canada). Waist circumference was determined from the CT image at the level of L4-L5, which approximates the level of the iliac crest. A contiguous series of 5 to $7 \mathrm{CT}$ images between the L4-L5 to L3-L4 vertebral disc spaces for each subject were analyzed for determination of abdominal subcutaneous and visceral fat. The AT volumes were calculated using a truncated pyramid method as described previously (33). Adipose tissue volumes (liters) were converted to mass units $(\mathrm{kg})$ by multiplying the volumes by the assumed constant density for fat $(0.92 \mathrm{~kg} / \mathrm{L})$. Adipose tissue areas $\left(\mathrm{cm}^{2}\right)$ were computed using an attenuation range of -190 to -30 Hounsfield units. Visceral AT was determined by delineating the intraabdominal cavity at the innermost aspect of the abdominal and oblique wall musculature and the anterior aspect of the vertebral body. Abdominal subcutaneous AT area was defined as the area of adipose tissue between the skin and the outermost aspect of the abdominal muscle wall. The deep and superficial depots of the abdominal subcutaneous AT were identified for a single image at the level of L4-L5 using the subcutaneous fascia.

We have previously reported the inter-observer reliability error for VAT and ASAT measurement for two observers' analyses of the same L4-L5 image $(n=40)$ to be $\sim 3 \%$ and $\sim 1 \%$, respectively (214).

CT is capable of differentiating tissues on the basis of their attenuation characteristics, which are a function of tissue density and chemical composition. A normal liver is usually denser and consequently has a higher attenuation value than the 
spleen. Therefore a lower mean liver attenuation value relative to that of the spleen is an indication of fatty infiltration of the liver (130). Liver fat was represented as both the liver attenuation (CTL, Hounsfield units (HU)) by itself, as well as the ratio of the liver to spleen (CTS) attenuation values (CTL/CTS) (130, 140). CTL and CTS were calculated using the average attenuation values for two regions of interest within each organ obtained from a single CT image that clearly displayed both the liver and spleen. The regions of interest were consistently placed in the parenchyma of the right lobe of the liver and in a similar region within the spleen, being careful to avoid blood vessels, artifacts, and other areas of inhomogeneity.

\section{Statistical analysis}

Independent sample t-tests and chi-square tests were used to determine group differences in subject characteristics. An unmatched case-comparison study with simple random sampling was used to identify the comparison subjects to estimate the association between independent abdominal fat depots and all-cause mortality (221). Logistic regression was used to estimate the odds ratios which are expressed per standard deviation unit to facilitate comparisons of odds ratios between different adipose tissue depots. Age, follow-up time, the abdominal fat depots and liver fat (CTL or CTL/CTS) were entered in the models as continuous variables. Linear, quadratic and cubic terms were tested for each continuous independent predictor variable using a stepwise approach with a $P<0.1$ to enter and a $P<0.05$ to remain in the model. Self-reported smoking status (current, past, never), blood pressure (normal, high), cholesterol (normal, high), triglycerides (normal, high) and type 2 diabetes (yes, no) were entered as categorical 
covariates. The odds ratios and $95 \%$ confidence intervals are reported after adjustment for age and follow-up time (Model 1) and after further adjustment for all three fat measures (visceral fat, abdominal subcutaneous fat, and liver fat) (Model 2). The figures were plotted using the $5^{\text {th }}$ percentile of visceral fat (mass $-0.15 \mathrm{~kg}$ and area $-50 \mathrm{~cm}^{2}$ ) as the referent value. All statistical analyses were performed using SAS v8. 


\section{RESULTS}

Subject characteristics are shown in Table 1. The decedents were significantly older and had more visceral fat $(P<0.05)$ than the controls. The decedents also had a significantly higher prevalence of self-reported type 2 diabetes, high blood pressure and past smoking than the controls $(P<0.05)$.

The associations between the various fat depots and mortality are shown in Table 2. After adjustment for age and follow-up time (Model 1), waist circumference, liver fat, abdominal subcutaneous fat and visceral fat (Figure 1a+b) were significant predictors of mortality $(\mathrm{P}<0.05)$. In a model including all three fat measures (visceral, abdominal subcutaneous and liver fat), age, and length of follow-up (Model 2), only visceral fat mass (OR [95\% CI], 1.81 [1.04-3.14], $\mathrm{P}<0.05)$ and area (L4-L5, 1.69 [0.99-2.89], $\mathrm{P}=0.5$ ) was a significant predictor of mortality (Table 2, Figure 1c+d). These results remained similar when traumatic deaths $(n=7)$ were excluded, after controlling for past or present smoking status, body mass index, co-morbidities (ie. high blood pressure, high cholesterol, type 2 diabetes, and high triglycerides), or when examining premature mortality in a subgroup of men aged 40-65y (data not shown). 


\section{DISCUSSION}

In this study we demonstrated that visceral, abdominal subcutaneous and liver fat (ie. low liver attenuation) were all directly associated with higher risk of mortality in men. However, visceral fat alone independently predicted risk of mortality after adjustment for the other fat measures. Waist circumference was also directly associated with mortality; however, the association did not remain significant after adjustment for visceral and subcutaneous fat. It has been hypothesized that visceral fat would be directly related to mortality risk based on plausible biological mechanisms $(54,66,98,222)$, however, to our knowledge this is the first study to demonstrate an association of visceral fat with mortality where visceral fat was quantified directly by CT measurements.

Our findings suggest that the relationship between visceral fat and mortality is curvilinear. Inspection of Figure 1 reveals that a rather large amount of visceral fat ( $200 \mathrm{~cm}^{2}$ at the L4-L5 level) accumulation is required prior to observing a substantially elevated risk of mortality. This threshold is greater than the level of visceral fat ( 110 to $130 \mathrm{~cm}^{2}$ at L4-L5) thought to be associated with a disproportionate increase in metabolic risk in moderately aged men (222). It is likely that these observations are consistent. In other words, a longer follow-up period in our study may have revealed that at a younger age our sample of older men had levels of visceral fat in the order of $130 \mathrm{~cm}^{2}$ combined with increased metabolic risk factors. It is well established that visceral fat accumulation increases with advancing age (198).

To our knowledge, this is the first study to demonstrate that liver fat is directly associated with mortality risk. Stated differently, liver density was inversely associated with mortality demonstrating that the leaner the liver, the lower the risk of mortality. 
Although liver fat was not associated with mortality following adjustment for visceral and subcutaneous fat, the unique contribution of liver fat to morbidity and mortality remains to be firmly established. This notion is underscored by clinical observations demonstrating that liver fat is positively associated with insulin resistance $(93,98)$ and dyslipidemia $(54,98)$ independent of visceral fat $(54)$. A definitive measure of liver fat that is appropriate for use in clinical settings is unknown. However, it is noteworthy that in our study waist circumference was a significant correlate of liver fat (waist circumference versus liver fat, $\mathrm{r}=-0.59, \mathrm{P}<0.001$ ). This confirms previous observations (223) and sets the stage for further investigation to determine whether waist circumference can be a useful anthropometric surrogate of fatty liver.

It is clear that both visceral and liver fat are associated with numerous metabolic risk factors, which themselves are antecedents for morbidity and mortality $(9,54,224)$. Contrary to the originally proposed substrate-based mechanism, often termed the Portal Theory (62), excess release of proinflammatory and prothrombotic factors may be the mechanism that links visceral fat and metabolic risk $(69,225)$. The mechanisms that link liver fat per se with metabolic risk, in particular insulin resistance, is largely unknown (224). Emerging evidence does strongly suggest, however, that liver fat is related to insulin resistance independent of abdominal obesity $(54,93)$ and that reductions in liver fat via weight loss $(119,226)$ or thiazolidinedione treatment $(124)$ are related to corresponding reduction in insulin resistance.

The findings of this study provide support for the recommendation that visceral fat should be a primary target of therapeutic strategies designed to reduce obesity related morbidity and mortality. It is encouraging therefore that visceral fat is substantially 
reduced in response to diet- or exercise-induced weight loss independent of age and gender $(11,12)$. It is generally reported that weight loss in the order of $10 \%$ is associated with a corresponding reduction in visceral fat that approximates $35 \%$ (227). Although routine measurement of visceral fat is not yet feasible, it is established that waist circumference is a strong correlate of visceral fat (228) and more importantly, that reductions in waist circumference are related to corresponding reductions in visceral fat in a dose-response manner $(13,228)$. Combined with our finding that waist circumference was directly associated with mortality (Figure 1), these observations support the recommendation that waist circumference should be a routine measure in clinical practice to characterize health risk, and of equal importance, to follow the success of strategies designed to reduce obesity and related co-morbid conditions.

Limitations of this study include the use of a relatively small opportunistic sample of men who had acquired abdominal CT images at the Cooper Institute. The men in this study are predominantly white, and from a middle-to-upper class population. This limits the generalizability of the results of our study, but should not affect the internal validity. Indeed, the homogeneity of our study group on socioeconomic factors is a benefit because it reduces the likelihood of confounding by these factors. Simple random sampling from our overall cohort to identify comparison subjects resulted in a large age difference between decedents and comparison participants. This is similar to substantial differences between decedents and survivors seen in prospective investigations; and, as in prospective studies, this difference is accounted for in the multivariable statistical model used in our primary analyses (221). The follow-up time in our study was relatively short and many of the decedents had morbid conditions (ie. hypertension, type 2 diabetes, etc.) 
upon entry to the study ( $55 \%$ of decedents versus $26 \%$ of controls). Thus, some of the deaths could be attributed to preexisting disease and may represent the conduit by which obesity lead to increased mortality. Although we relied on self-reported diagnosis for diabetes, hypercholesterolemia, and hypertension, the accuracy of self-reported diagnoses in this cohort has been previously demonstrated. For example, we have demonstrated that in this cohort self-report hypertension had a sensitivity of $98 \%$, and specificity of 99\% (229). Nevertheless, adjustment for the differences in the existence of the comorbidities at baseline did not change the association between visceral fat and mortality.

The strengths of this study include the use of a multi-image CT protocol for measurement of abdominal fat which allowed us to determine subcutaneous and visceral fat mass for a large portion of the abdomen, substantially reducing the potential influence of measurement site (e.g., L4-L5 or L3-L4 intervertebral sites) on our estimate of visceral fat. It is noteworthy, however, that the risk of mortality associated with visceral fat mass and area at the L4-L5 level were not different (Table 2).

Visceral fat is a significant predictor of mortality in men after adjustment for age, follow-up time, subcutaneous fat and liver fat. These findings underscore the importance of setting abdominal obesity as a primary target for obesity reduction and consequently, the need to educate health care practitioners on the importance of routine measurement of visceral fat. Although we measured visceral fat directly by CT scans in this study, waist circumference provides a reasonable approximation of visceral fat in clinical settings. 


\section{ACKNOWLEDGEMENTS}

The authors would like to extend a special thanks to Elisa Priest for her work related to the management of the CT data. This research was supported in part by research grants from the National Institutes of Health to Steven N. Blair (AG06945) and Milton Z. Nichaman and Michael J. LaMonte (HL62508), and from the Canadian Institutes of Health Research to Robert Ross (MT13448). 


\section{Table 1: Subject Characteristics}

\begin{tabular}{|c|c|c|}
\hline & Control & Decedents \\
\hline $\mathrm{N}$ & 194 & 97 \\
\hline Follow-up Time, yr $\dagger$ & $1.8(1.2)$ & $2.9(1.0) *$ \\
\hline Age, yr $\dagger$ & $51.8(9.3)$ & $65.6(11.4) *$ \\
\hline Body Mass Index, $\mathrm{kg} / \mathrm{m}^{2}+t$ & $26.8(3.2)$ & $26.4(4.8)$ \\
\hline Waist Circumference, $\mathrm{cm} \dagger$ & $94.8(9.2)$ & $96.7(11.2)$ \\
\hline Visceral Fat Mass, $\mathrm{kg} \dagger$ & $0.54(0.25)$ & $0.68(0.39) *$ \\
\hline Abdominal Subcutaneous Fat Mass, $\mathrm{kg} \dagger$ & $0.75(0.29)$ & $0.73(0.34)$ \\
\hline Visceral Fat Area (L4-L5), $\mathrm{cm}^{2} \dagger$ & $141.3(61.1)$ & $174.2(96.6) *$ \\
\hline Abdominal Subcutaneous Fat Area (L4-L5), $\mathrm{cm}^{2} \dagger$ & $226.1(86.2)$ & $217.4(96.6)$ \\
\hline Mean Liver Attenuation, $\mathrm{HU} \uparrow$ & $58.3(10.7)$ & $55.8(12.0)$ \\
\hline $\mathrm{CTL} / \mathrm{CTS} \dagger$ & $1.12(0.19)$ & $1.17(0.23)$ \\
\hline High Blood Pressure, \% & 14.9 & $30.0 *$ \\
\hline High Cholesterol, \% & 25.8 & 26.8 \\
\hline High Triglycerides, \% & 13.4 & 16.5 \\
\hline Type 2 Diabetes, $\%$ & 2.1 & $11.3 *$ \\
\hline Current Smoker, \% & 12.4 & 12.4 \\
\hline Past Smoker, \% & 24.7 & $40.2^{*}$ \\
\hline
\end{tabular}

$\mathrm{CTL} / \mathrm{CTS}=$ Ratio of liver to spleen attenuation values

* Significantly different from Control at $\mathrm{P}<0.05$.

$\dagger$ Values are presented as mean (SD)

$\$$ Decedents $(n=73)$, Control $(n=97)$ 
Table 2: Odd-Ratios for All-Cause Mortality

\begin{tabular}{|c|c|c|c|c|c|c|c|c|}
\hline & \multicolumn{4}{|c|}{ Model 1} & \multicolumn{4}{|c|}{ Model 2} \\
\hline & $\boldsymbol{\beta}$ & SE & OR $(95 \% \text { CI })^{*}$ & $P$ Value & $\boldsymbol{\beta}$ & SE & OR $(95 \% \text { CI })^{*}$ & $P$ Value \\
\hline$\overline{\text { Visceral Fat Mass }}^{3}, \mathbf{k g}^{\dagger}$ & 0.85 & 0.29 & $1.83(1.23-2.73)$ & 0.003 & 0.86 & 0.41 & $1.81(1.04-3.14)$ & 0.04 \\
\hline Subcutaneous Fat Mass, kg & 1.20 & 0.58 & $1.44(1.02-2.03)$ & 0.04 & -0.02 & 0.78 & $0.99(0.63-1.58)$ & 0.98 \\
\hline Visceral Fat ${ }^{3}$ (L4-L5), $\mathrm{cm}^{2 \dagger}$ & $5.0 \times 10^{-8}$ & $1.9 \times 10^{-8}$ & $1.76(1.16-2.67)$ & 0.008 & $4.7 \times 10^{-8}$ & $2.4 \times 10^{-8}$ & $1.69(0.99-2.89)$ & 0.05 \\
\hline Subcutaneous Fat (L4-L5), $\mathrm{cm}^{2}$ & 0.003 & 0.002 & $1.31(0.93-1.85)$ & 0.15 & -0.002 & 0.003 & $0.85(0.53-1.36)$ & 0.49 \\
\hline Waist Circumference $^{3}, \mathrm{~cm}^{\dagger}$ & $1.19 \times 10^{-6}$ & $5.89 \times 10^{-7}$ & $1.41(1.01-1.98)$ & 0.04 & $-1.7 \times 10^{-6}$ & $1.53 \times 10^{-6}$ & $0.60(0.25-1.44)$ & 0.26 \\
\hline Mean Liver, HU & -0.04 & 0.01 & $0.64(0.46-0.87)$ & 0.005 & -0.01 & 0.02 & $0.87(0.56-1.37)$ & 0.55 \\
\hline CTL/CTS & -1.37 & 0.82 & $0.76(0.54-1.05)$ & 0.10 & 1.13 & 1.69 & $1.26(0.64-2.48)$ & 0.50 \\
\hline
\end{tabular}

*The odds-ratios for mortality in the table are expressed per standard deviation for each variable.

$\mathrm{CTL} / \mathrm{CTS}=$ Ratio of liver to spleen attenuation values

Model 1: Control for age + follow-up time

Model 2: Control for age, follow-up time, abdominal subcutaneous fat, visceral fat and liver fat

$\uparrow$ Cubic transformation used in the analyses 
Figure 1 - The odds-ratio for mortality with increasing visceral fat mass $(A+C)$ and $\operatorname{area}(\mathbf{B}+\mathbf{D})$.
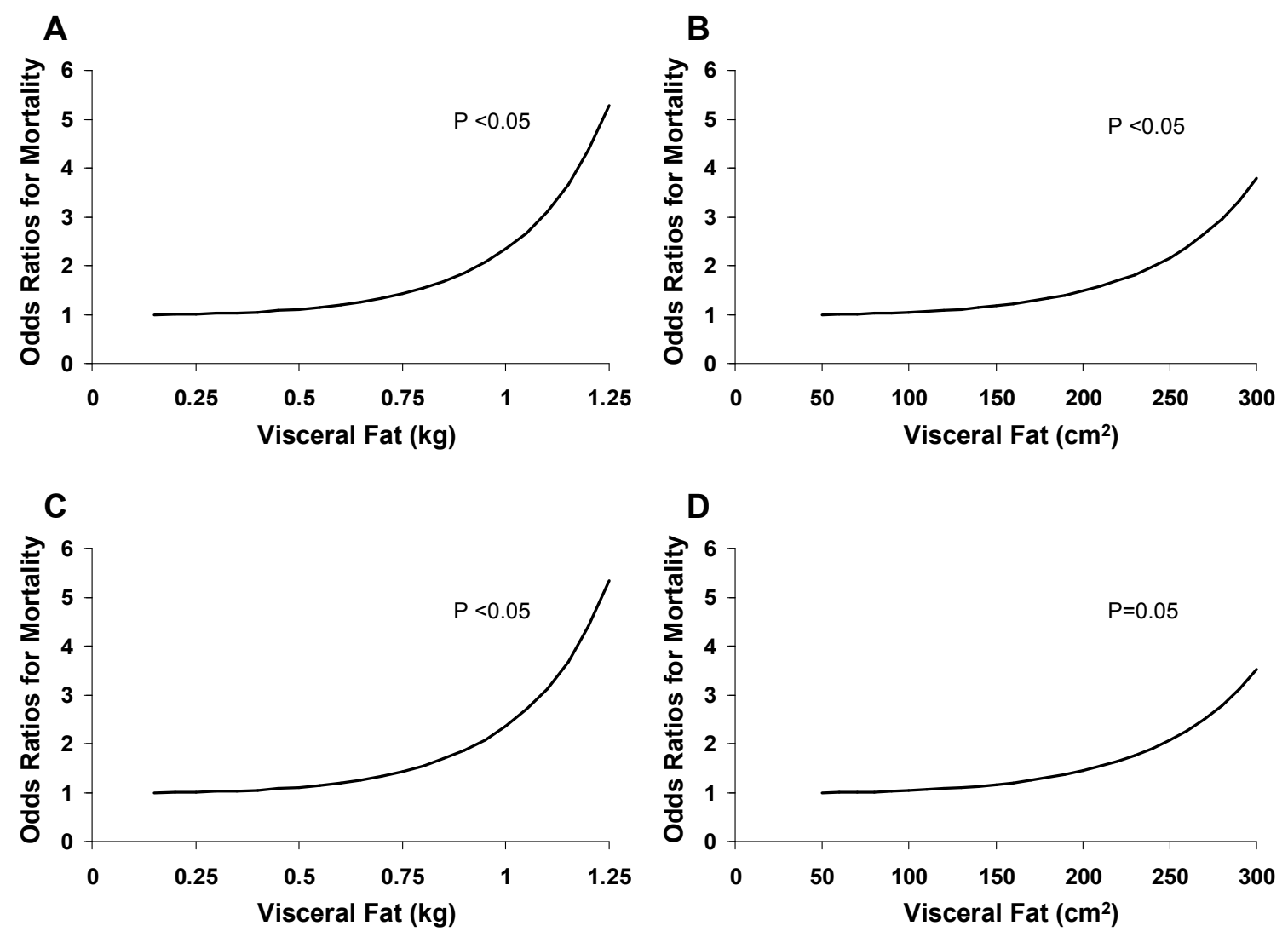

The odds-ratio for mortality with increasing visceral fat mass (A) and area (B), after control for age and follow-up time. The odds-ratio for mortality with increasing visceral fat mass (C) and area (D), after control for age, follow-up time, liver fat and abdominal subcutaneous fat. The $5^{\text {th }}$ percentile of visceral fat (mass $-0.15 \mathrm{~kg}$ and area $-50 \mathrm{~cm}^{2}$ ) was used as the referent value. 


\section{Chapter 6. Manuscript 4}

The co-authors of this manuscript are Steven N. Blair, Timothy S. Church and Robert Ross. This research was supported in part by research grants from the Canadian Institutes of Health Research to Robert Ross (MT13448) and the National Institutes of Health to Steven N. Blair (AG-06945). 
Influence of Exercise Dose on Changes in Abdominal Obesity in Postmenopausal Women - The DREW Study 


\begin{abstract}
Objective: To determine the effect of aerobic exercise dose (energy expenditure) on waist circumference and abdominal fat.

Research Methods and Procedures: Sedentary, overweight/obese postmenopausal women $(n=424)$ aged 45-75 years were randomly assigned to a control group or one of three aerobic exercise groups that exercised at a moderate intensity $\left(50 \% \mathrm{VO}_{2 \max }\right), 3-4$ days/week and expended 4-, 8- or 12-kcal/kg body weight/week for 6-months. Waist and hip circumference, and body weight was assessed in 424 women. Abdominal (visceral) fat, liver fat, thigh subcutaneous fat, and thigh muscle composition was assessed using computed tomography in a sub-sample of 87 women.
\end{abstract}

Results: Analysis of the total sample $(n=424)$ revealed a significant reduction in waist circumference in the exercise groups relative to control $(\mathrm{P}<0.05)$, with no differences between the exercise groups $(\mathrm{P}>0.10)$. Conversely, there were no significant reductions in body weight or the waist-to-hip ratio $(\mathrm{P}>0.10)$. Further, the reduction in waist circumference remained significant independent of changes in body weight, ethnicity or hormone replacement therapy use. In the sub-sample $(n=87)$, there were no significant $(\mathrm{P}>0.05)$ group differences in waist circumference or any of the CT measures (visceral fat, abdominal subcutaneous fat, liver fat, thigh skeletal muscle mass or composition or thigh subcutaneous fat).

Discussion: Aerobic exercise at or $50 \%$ below or above current guidelines is associated with significant reductions in waist circumference independent of change in body weight. However, the amount of exercise performed was not associated with reductions in waist circumference in a dose dependent manner. 
Key Words: Liver fat, abdominal fat, computed tomography, thigh muscle composition, thigh subcutaneous fat. 


\section{INTRODUCTION}

Abdominal obesity as measured by waist circumference is a strong predictor of morbidity $(9,203,204)$ and mortality $(110,156,230,231)$ and thus is a target for therapeutic interventions designed to reduce obesity-related health risk. While it is generally accepted that 30 minutes of moderate intensity exercise on all or most days of the week ( 150 minutes/week) is sufficient to elicit substantial benefit across a wide range of health outcomes (15), it is unclear whether this exercise dose is sufficient to elicit significant reductions in abdominal obesity $(168,173-177)$. Whereas some randomized controlled trials report significant reductions in waist circumference (173$175)$, some do not $(168,176,177)$. The discrepant findings may be partially explained by small sample size $(176,177)$ or increased dietary intake to maintain body weight $(168)$. Studies that prescribe $\sim 150$ minutes/week of moderate intensity exercise generally report a 1 to $3 \mathrm{~cm}$ reduction in waist circumference relative to the respective control group (173175). However, studies that prescribe exercise doses of less than the recommendation ( $\sim 100$ minutes/week) also show reductions in waist circumference in the order of $2 \mathrm{~cm}$ $(178,179)$, whereas studies that prescribe more than the recommendations $(\sim 450$ minutes/week) show substantially larger reductions in waist circumference in the order of $6 \mathrm{~cm}(11,12)$. It is difficult to infer from these studies that the magnitude of the waist circumference reduction is dependent upon the exercise time or energy expenditure prescribed as these studies differed in terms of the exercise intensity (40 to $80 \% \mathrm{VO}_{2 \max }$ ), length of the intervention (2 to 12 months), study cohort characteristics (men vs women, younger vs older, T2D vs no T2D, etc.), exercise modality (walking, biking, etc.) or increased dietary intake $(168,174)$; all of which would influence the exercise energy 
expenditure, net energy balance and ultimately the expected reduction in waist circumference. No study to date has simultaneously examined the influence of multiple exercise doses (ie. energy expenditure) alone on reductions in abdominal obesity using a rigorously controlling study design.

We examined the influence of exercise dose (energy expenditure) at 50\% below, $50 \%$ above and at the current exercise recommendations of the U.S. Public Health Service (15), and tested the hypothesis that the postmenopausal women would reduce waist circumference with increasing exercise energy expenditure in a dose-dependent manner. 


\section{RESEARCH DESIGN AND METHODS}

\section{Subject Criteria}

Subjects included a sample of 424 sedentary, postmenopausal women aged 45-75 years of age, initially recruited for participation in a 6-month exercise intervention study conducted at the Cooper Clinic in Dallas, Texas. The details of this intervention have been described previously (232). Postmenopausal status was defined as no natural menses for 2 years and a serum follicle stimulating hormone level $>40 \mathrm{mIU} / \mathrm{ml}$. Hormone replacement therapy was allowed if the subject had been on a stable dose for at least 6 months. Inclusion criteria required that the subjects be moderately overweight or obese (body mass index of 25 to $34.9 \mathrm{~kg} / \mathrm{m}^{2}$ ). Exclusion criteria included persons with history of diabetes mellitus, hyperlipidemia (total cholesterol $\geq 240 \mathrm{mg} / \mathrm{dl}$ and LDL-C $\geq 160$ mg/dl, or triglycerides $>300$ ), cardiovascular disease, stroke, cancer, Stage II or III hypertension or recent excessive weight loss. Subjects taking medication to treat hypertension were also excluded. All subjects gave their informed written consent prior to participation according to the ethical guidelines of The Cooper Institute Institutional Review Board, and the study was reviewed and approved annually.

\section{Clinical Examination}

During the 2 to 3 week pre-randomization period, study participants completed a series of screenings and assessments wherein all baseline measures were taken. The assessments included several questionnaires on demographic factors, medical history, and health habits (alcohol use, cigarette smoking habits, physical activity, and medication use), anthropometric and blood pressure measurements, blood chemistry analyses, resting 
ECG, standardized maximal exercise tests and a general physical examination. Body weight and height were measured using a standard physician's scale and stadiometer and body mass index was calculated (weight in kilograms / height in meters squared). Waist circumference was measured at the level of the umbilicus. All assessments were conducted by trained personnel who were blinded to group assignment.

\section{CT Assessment}

Axial images of the abdominal and thigh region were obtained using an electron beam computed tomography on a subset of 87 women, beginning partway through the study (CT, Imatron, General Electric, Milwaukee, WI) using a standard protocol (213). Subjects were examined in a supine position with their arms extended above their heads. Approximately 40 contiguous transverse images ( $6 \mathrm{~mm}$ thickness) were acquired in the abdomen spanning from the mid-region of the iliac crest to the caudal region of the heart. Similarly, 50 contiguous images were acquired in the thigh region beginning just distal to the patella to approximately $18 \mathrm{~cm}$ above the patella. Images were obtained using $130 \mathrm{kV}$ and $630 \mathrm{~mA}$ with a $48 \mathrm{~cm}$ field of view and a $512 \times 512$ matrix. The CT data obtained in Dallas was transferred electronically to the laboratory in Kingston for analysis using specially designed image analysis software (Tomovision, Inc., Montreal, Canada). To minimize bias, one technician conducted all images analyses while blinded to group assignment.

Abdominal AT areas $\left(\mathrm{cm}^{2}\right)$ were computed using an attenuation range of -190 to $30 \mathrm{HU}$ (233). Visceral AT was determined by delineating the intra-abdominal cavity at the innermost aspect of the abdominal and oblique wall musculature and the posterior 
aspect of the vertebral body. Abdominal subcutaneous AT area was defined as the area of adipose tissue between the skin and the outermost aspect of the abdominal muscle wall. Abdominal AT volumes $\left(\mathrm{cm}^{3}\right)$ were determined from approximately 21 contiguous images from L1 to L5, which was the largest region available for all subjects pre and post intervention. The volume $\left(\mathrm{cm}^{3}\right)$ of AT was calculated as the sum of all partial AT tissue volumes $\left(\mathrm{cm}^{3}\right)$ within the region bordered by L1 and L5.

Liver fat was determined using the ratio of the liver to spleen attenuation values (CTL/CTS) $(130,140)$. CTL and CTS were calculated using the average attenuation value of all the pixels within each organ. The borders of the organs were identified manually using specialized imaging software (Tomovision, Inc., Montreal, Canada) and procedures which are described in detail elsewhere (234). To facilitate understanding, Liver Fat Scores (LFS) were calculated by taking the inverse of CTLCTS (1/CTLCTS), to create a positive relationship between LFS and liver fat.

Thigh tissue composition was determined using the mean attenuation values of the muscle at the mid-thigh using 11 contiguous images spanning from 12 to $18 \mathrm{~cm}$ proximal to the patella. Skeletal muscle attenuation in the thigh was defined for each subject as the mean attenuation value from all pixels within the range of 0-100 $\mathrm{HU}(235$, 236). Skeletal muscle was subdivided into the low- and high-density muscle. Lowdensity muscle was defined as muscle within the range of 0-30 $\mathrm{HU}$, and high-density muscle was defined as muscle within the range of 31-100 HU. As lipid is characterized by negative attenuation values, mean muscle attenuation is negatively correlated with muscle lipid content. These methods have been validated and previously described elsewhere (109). The test-retest coefficient of variation for two CT scans is $<1 \%$ (109). 
Skeletal muscle and adipose tissue volumes (liters) were converted to mass units (kg) by multiplying the volumes by the assumed constant density for skeletal muscle $(1.04 \mathrm{~kg} / \mathrm{L})$ and fat $(0.92 \mathrm{~kg} / \mathrm{L})(237)$.

\section{Maximal Oxygen Uptake Measurements}

Cardiorespiratory fitness was assessed using a graded maximal cycle protocol using a Sensormedics Ergo-Metrics 800S cycle ergometer. Peak oxygen uptake was calculated as the average of the peak oxygen uptake from two maximal exercise tests, measured before and after the intervention using a Sensormedics 2900 Metabolic Measurement Cart. On average, the tests were separated $6.0 \pm 4.5$ days prior to the intervention, and $5.4 \pm 4.3$ days after the intervention.

\section{Exercise Intervention}

After the 2-3 week run-in period, the women were randomly assigned to a control group or one of three exercise groups that differed in energy expenditure for 6-months. The intervention has been described previously (232). Briefly, the women exercised 3 to 4 times per week at a target training intensity of $50 \%$ of $\mathrm{VO}_{2 \max }$. We estimated that in this population, moderate intensity exercise $\left(50 \% \mathrm{VO}_{2 \max }\right)$ for 30 minutes, 5 days a week translates to approximately $8 \mathrm{kcal} / \mathrm{kg}$ body weight/week $(238,239)$. As such, the exercise

groups expended 4-, 8- or 12-kcal/kg body weight/week over the intervention alternating training sessions on a cycle ergometer and treadmill. All exercise sessions were by appointment and performed under the supervision of trained personnel at the Cooper 
Institute. Successful adherence was defined as completing $90 \%$ of the total amount of exercise energy expenditure prescribed.

\section{Statistical Analysis}

Group data are presented as means $\pm \mathrm{SD}$. Age, anthropometric measures, cardiorespiratory fitness, abdominal AT, skeletal muscle variables, and liver fat were entered in the model as continuous covariates. Ethnicity and hormone replacement use was entered in the model as categorical covariates. Baseline group differences in age, adiposity and fitness were assessed using a one-way analysis of variance. Analyses of continuous outcome measures were assessed using ANCOVA models of the 6-month scores adjusted for baseline values, with and without control for age, ethnicity and hormone replacement therapy. Treatment effects were summarized as least-square adjusted means. Analyses of dose-response trends were based on ordinary least-square regression of endpoints across the three exercise groups. All statistical analyses were performed using SAS v9. 


\section{RESULTS}

Subject characteristics are shown in Table $\mathbf{1}+\mathbf{2}$. There were no between group differences for any of the baseline characteristics in the full sample and sub-sample $(\mathrm{P}>0.05)$.

\section{Changes in Waist Circumference, Waist-to-Hip Ratio and Body Weight}

Analysis of the total sample $(n=424)$ revealed a significant reduction in waist circumference in the exercise groups relative to control $(\mathrm{P}<0.05)$, with no differences between the exercise groups $(\mathrm{P}>0.10$, Figure 1a). Conversely, there were no significant reductions in hip circumference, waist-to-hip ratio or body weight $(\mathrm{P}>0.10$, Figure $\mathbf{1 b}+\mathbf{c})$. Further, the reduction in waist circumference remained significant independent of changes in body weight $(\mathrm{P}<0.05)$. In the sub-sample $(\mathrm{n}=87)$, changes in waist circumference, hip circumference, waist-to-hip ratio and body weight did not reach statistical significance $(\mathrm{P}>0.10)$.

\section{Changes in the CT Measures}

In the sub-sample $(\mathrm{n}=87)$, there were no significant group differences in any of the CT measures (visceral fat, abdominal subcutaneous fat, liver fat, thigh skeletal muscle mass or composition or thigh subcutaneous fat) (Figure 2 and Table 3). These observations remained true independent of age, ethnicity or hormone replacement use. 


\section{DISCUSSION}

The primary finding of this study is that physical activity performed at or $50 \%$ below or above the current guidelines for attaining health benefits is associated with significant reductions in waist circumference independent of weight loss. However, the exercise-induced reduction in waist circumference was not dose dependent.

Waist circumference is a powerful predictor of morbidity $(9,203,204)$ and mortality $(110,156,230,231)$, and thus it is important to develop effective therapeutic strategies for reducing waist circumference. Consistent with previous observations (11, $12,24,163)$, we report that modest levels of physical activity is associated with significant reductions in abdominal obesity independent of weight loss. This has important clinical implications when one considers that this intervention would have been considered a failure had BMI or body weight been the sole indicator treatment success. Indeed, body weight alone was insensitive to significant reduction in abdominal obesity. This finding is particularly important for individuals who are resistant to weight loss, as reductions in waist circumference may provide additional motivation and positive reinforcement. These observations emphasize the importance of acquiring measures of waist circumference when determining the efficacy of treatment strategies designed to manage obesity.

In accordance with previous studies $(174,178)$, we did not observe a significant change in WHR despite significant reductions in waist circumference. Although WHR is a strong predictor of morbidity $(7,240)$ and mortality $(240)$, WHR is limited in its utility to assess change in abdominal obesity because obesity reduction is generally associated with simultaneous reductions in waist and hip circumference and hence, no change in 
WHR $(35,228,241,242)$. Thus waist circumference alone is superior to WHR when determining the efficacy of strategies designed to reduce obesity $(35,228,242)$ and related co-morbid conditions (243).

Although it is established that exercise is associated with corresponding decreases in abdominal obesity $(11,12,24,244)$, absent are studies that systematically examine the influence of exercise dose (energy expenditure) on reductions in abdominal obesity in the absence of dietary modification. In this study we did not observe the expected dose response relationship between reductions in waist circumference and exercise energy expenditure. Indeed, the reductions in waist circumference across the exercise groups were not different $(\sim 2$ to $3 \mathrm{~cm})$ despite careful control to ensure frank differences in exercise energy expenditure (311 to $843 \mathrm{kcal} /$ week) between the groups over the duration of the study. Although we had excellent compliance to the exercise intervention, there was substantial inter-individual variation in the waist circumference response with exercise observed within each group. This agrees with Slentz et al. (174), who did not observe a difference between low and high doses of vigorous physical activity (12 vs. 20 miles/wk at $65-80 \% \mathrm{VO}_{2 \text { peak }}$ ) on waist circumference or visceral adiposity. However, this study may have been confounded because subjects were instructed to maintain their body weight. In this study, it is unclear whether individuals in the group with the highest exercise dose (12-kcal/kg/wk) increased their caloric intake and/or whether the lowest exercise dose decreased their caloric intake. Alternatively, it is also plausible that individuals in the highest exercise dose had compensatory decreases in their energy expenditure throughout the rest of the day, thus negating their exercise energy expenditure $(245,246)$. 
In this study exercise was not associated with changes in visceral fat, or fat deposition within the liver, pancreas or muscle. We were likely limited by sample size in the sub-sample as reductions in waist circumference did not reach statistical significance despite similar magnitudes of change compared to the full cohort. Post hoc analyses revealed that in order to achieve $80 \%$ power at least 16 to subjects per group (only 11 in the control group) would be required to achieve statistical significance. Although the independent contribution of these fat depots in the development of disease is unclear, these fat depots have been associated with insulin resistance $(11,12,93,99,111)$, dyslipidemia $(93,96)$ and hypertension $(93)$. Previous studies $(174-176,190)$ that have prescribed 75 to 225 minutes/week of physical activity generally report only modest if any reductions in visceral fat $(\sim 6$ to $7 \%)(174,175)$. The efficacy of exercise training as a treatment strategy for liver, pancreas or muscle fat infiltration and related morbidities, without modifications to dietary intake is less clear as there are only a few studies to date that have examined this issue with mixed results $(185,247)$. Clearly additional research is required to define the dose-response relationship between exercise (energy expenditure or intensity) and these fat depots.

Limitations of this study warrant mention. First, these observations are only applicable to relatively younger postmenopausal who underwent a stringent supervised exercise intervention with very high compliance. Although we did not control for changes in dietary habits over the course of the study, all groups received the same dietary counseling, and thus the effect should be consistent across all groups. Second, in this study we differentiated exercise doses by modifying energy expenditure at a moderate intensity. Unknown is whether we would have observed a dose-response 
relationship had we used a higher exercise intensity or a larger range of exercise energy expenditures. Further, it is unclear whether there is a dose-response relationship across exercise intensities if energy expenditure or time held constant. Clearly, more work is needed to further define the dose response relationship between exercise and reductions in abdominal obesity, visceral fat, and other fat depots.

In conclusion, modest levels of physical activity consistent with current guidelines for obtaining health benefit across a wide range of health outcomes, is also associated with modest reductions in waist circumference independent of weight loss. Although the exercise-induced reduction in waist circumference was not dose dependent, our findings suggest physical activity levels that are half the current recommendations may be useful for management of abdominal obesity. 


\section{ACKNOWLEDGEMENTS}

The authors would like to extend a special thanks to Elisa Priest for her work related to the management of the data, and the study participants for their time and dedication. This research was supported in part by research grants from the National Institutes of Health to Steven N. Blair (AG06945) and from the Canadian Institutes of Health Research to Robert Ross (MT13448). 
Table 1: Baseline Subject Characteristics for the Entire Cohort

\begin{tabular}{|c|c|c|c|c|}
\hline & Control & $4 \mathrm{kcal} / \mathrm{kg} /$ week & $8 \mathrm{kcal} / \mathrm{kg} /$ week & $12 \mathrm{kcal} / \mathrm{kg} /$ week \\
\hline$\overline{\mathbf{N}}$ & 94 & 145 & 89 & 96 \\
\hline Age (y) & $57.2 \pm 5.9$ & $58.0 \pm 6.5$ & $56.8 \pm 6.6$ & $56.7 \pm 6.5$ \\
\hline Weight (kg) & $86.0 \pm 12.5$ & $83.7 \pm 11.4$ & $85.4 \pm 12.8$ & $83.4 \pm 11.2$ \\
\hline $\operatorname{BMI}\left(\mathrm{kg} / \mathrm{m}^{2}\right)$ & $32.2 \pm 3.9$ & $31.5 \pm 3.7$ & $32.3 \pm 4.1$ & $31.1 \pm 3.5$ \\
\hline Waist (cm) & $102.7 \pm 12.0$ & $100.2 \pm 11.1$ & $102.2 \pm 11.9$ & $99.1 \pm 11.2$ \\
\hline Waist-to-Hip Ratio & $0.88 \pm 0.08$ & $0.88 \pm 0.07$ & $0.88 \pm 0.07$ & $0.87 \pm 0.08$ \\
\hline $\mathrm{VO}_{2 \text { peak }}(\mathrm{L} / \mathrm{min})$ & $1.32 \pm 0.29$ & $1.28 \pm 0.25$ & $1.25 \pm 0.25$ & $1.32 \pm 0.25$ \\
\hline Exercise EE (kcal) * & & $7471 \pm 1892$ & $15024 \pm 2418$ & $20224 \pm 3377$ \\
\hline
\end{tabular}

Values are presented as mean $\pm \mathrm{SD}$. No significant baseline differences $(\mathrm{P}>0.05)$.

* Exercise Energy Expenditure (EE) significantly different between groups $(\mathrm{P}<0.01)$. 
Table 2: Baseline Subject Characteristics for the Sub-Sample with CT Data

\begin{tabular}{|c|c|c|c|c|}
\hline & Control & $4 \mathrm{kcal} / \mathrm{kg} /$ week & $8 \mathrm{kcal} / \mathrm{kg} /$ week & $12 \mathrm{kcal} / \mathrm{kg} /$ week \\
\hline $\mathbf{N}$ - Sub-sample & 11 & 28 & 19 & 29 \\
\hline Age (y) & $57.1 \pm 4.7$ & $58.8 \pm 6.9$ & $58.5 \pm 6.2$ & $58.1 \pm 6.3$ \\
\hline $\operatorname{BMI}\left(\mathrm{kg} / \mathrm{m}^{2}\right)$ & $32.1 \pm 4.2$ & $31.8 \pm 3.8$ & $32.2 \pm 3.9$ & $30.7 \pm 3.3$ \\
\hline Waist (cm) & $103.0 \pm 15.2$ & $102.4 \pm 11.4$ & $101.4 \pm 10.4$ & $102.6 \pm 9.8$ \\
\hline Waist-to-Hip Ratio & $0.86 \pm 0.11$ & $0.87 \pm 0.07$ & $0.88 \pm 0.08$ & $0.89 \pm 0.09$ \\
\hline Exercise EE (kcal) * & & $8105 \pm 1172$ & $15420 \pm 1917$ & $19572 \pm 3273$ \\
\hline \multicolumn{5}{|l|}{ CT Data } \\
\hline Visceral AT (kg) & $1.49 \pm 0.59$ & $1.66 \pm 0.75$ & $1.56 \pm 0.54$ & $1.81 \pm 0.64$ \\
\hline Visceral AT@L4-L5 ( $\left.\mathrm{cm}^{2}\right)$ & $143.2 \pm 62.2$ & $148.4 \pm 61.1$ & $142.2 \pm 48.7$ & $163.2 \pm 50.7$ \\
\hline $\operatorname{ASAT}(\mathrm{kg})$ & $3.84 \pm 1.33$ & $3.98 \pm 1.17$ & $3.63 \pm 0.81$ & $3.76 \pm 1.18$ \\
\hline ASAT@L4-L5 ( $\left.\mathrm{cm}^{2}\right)$ & $428.4 \pm 152.4$ & $465.2 \pm 146.8$ & $406.9 \pm 102.5$ & $420.5 \pm 108.3$ \\
\hline Liver Fat Score & $0.92 \pm 0.49$ & $0.88 \pm 0.35$ & $0.83 \pm 0.23$ & $0.81 \pm 0.18$ \\
\hline Liver Attenuation (HU) & $58.5 \pm 16.1$ & $57.6 \pm 14.5$ & $58.2 \pm 11.5$ & $60.0 \pm 10.4$ \\
\hline Pancreas Attenuation (HU) & $48.1 \pm 6.2$ & $43.8 \pm 11.2$ & $44.0 \pm 10.6$ & $44.0 \pm 11.2$ \\
\hline Thigh SAT (kg) & $1.72 \pm 0.40$ & $1.69 \pm 0.42$ & $1.72 \pm 0.48$ & $1.66 \pm 0.44$ \\
\hline Thigh Muscle (kg) & $1.36 \pm 0.22$ & $1.33 \pm 0.23$ & $1.34 \pm 0.19$ & $1.25 \pm 0.15$ \\
\hline Thigh LDM (kg) & $0.26 \pm 0.07$ & $0.23 \pm 0.05$ & $0.23 \pm 0.07$ & $0.22 \pm 0.05$ \\
\hline Thigh HDM (kg) & $1.11 \pm 0.18$ & $1.10 \pm 0.22$ & $1.10 \pm 0.17$ & $1.03 \pm 0.16$ \\
\hline Thigh Muscle (HU) & $53.6 \pm 4.3$ & $54.2 \pm 3.8$ & $54.4 \pm 4.4$ & $53.8 \pm 3.8$ \\
\hline
\end{tabular}

Values are presented as mean $\pm \mathrm{SD}$. No significant baseline differences $(\mathrm{P}>0.05)$.

* Exercise Energy Expenditure (EE) significantly different between groups $(\mathrm{P}<0.01)$. AT - Adipose Tissue; ASAT - Abdominal Subcutaneous AT; LDM - Low Density Muscle; HDM - High Density Muscle; 
Table 3: Changes in Subject Characteristics in the Sub-Sample with CT Data

\begin{tabular}{|c|c|c|c|c|}
\hline & Control & 4 kcal/kg/week & $8 \mathrm{kcal} / \mathrm{kg} /$ week & $12 \mathrm{kcal} / \mathrm{kg} /$ week \\
\hline Weight (kg) & $-1.0 \pm 3.0$ & $-1.6 \pm 4.0$ & $-2.8 \pm 4.4$ & $-1.3 \pm 2.8$ \\
\hline Waist (cm) & $2.9 \pm 7.7$ & $-2.6 \pm 7.3$ & $-0.6 \pm 7.0$ & $-2.9 \pm 8.0$ \\
\hline Waist-to-Hip Ratio & $0.06 \pm 0.07$ & $-0.01 \pm 0.07$ & $0.00 \pm 0.08$ & $-0.01 \pm 0.09$ \\
\hline \multicolumn{5}{|l|}{ CT Data } \\
\hline Visceral AT (kg) & $0.05 \pm 0.18$ & $-0.04 \pm 0.20$ & $-0.04 \pm 0.18$ & $-0.02 \pm 0.19$ \\
\hline ASAT (kg) & $-0.03 \pm 0.51$ & $-0.14 \pm 0.34$ & $-0.13 \pm 0.36$ & $-0.05 \pm 0.30$ \\
\hline Liver Attenuation (HU) & $-2.90 \pm 7.00$ & $0.60 \pm 6.33$ & $1.60 \pm 7.32$ & $0.79 \pm 6.39$ \\
\hline Pancreas Attenuation (HU) & $-2.66 \pm 6.07$ & $1.17 \pm 5.43$ & $1.70 \pm 4.82$ & $0.25 \pm 7.55$ \\
\hline Thigh LDM (kg) & $0.01 \pm 0.04$ & $-0.01 \pm 0.04$ & $0.01 \pm 0.06$ & $0.00 \pm 0.04$ \\
\hline Thigh HDM (kg) & $0.00 \pm 0.05$ & $0.01 \pm 0.05$ & $0.02 \pm 0.08$ & $0.02 \pm 0.07$ \\
\hline
\end{tabular}

Values are presented as mean $\pm \mathrm{SD}$. No significant effect of exercise as compared to the control group $(\mathrm{P}>0.05)$. 
Figure 1: Changes in anthropometric variables in response to 6 months of exercise training in postmenopausal women
Control
$\square 4 \mathrm{kcal} / \mathrm{kg} / \mathrm{wk}$ (75 min/wk)
$8 \mathrm{kcal} / \mathrm{kg} / \mathrm{wk}$ (150 $\mathrm{min} / \mathrm{wk})$
$12 \mathrm{kcal} / \mathrm{kg} / \mathrm{wk}$ (225 min/wk)
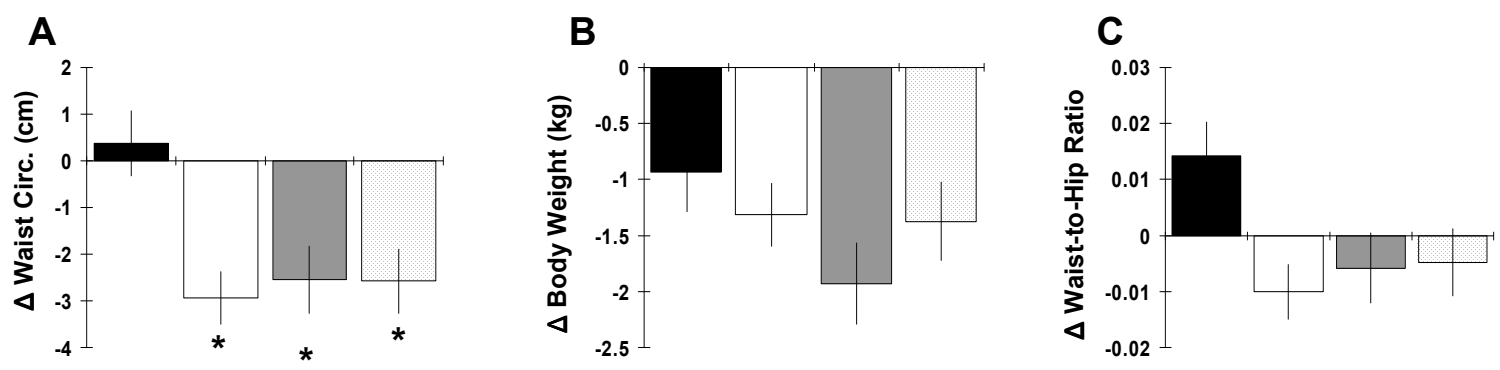

Change scores presented are least squared adjusted means $\pm \mathrm{SE}$, controlled for baseline values. $\mathrm{N}=424$. $*$ Significant difference from control $(\mathrm{P}<0.05)$. 
Figure 2: Changes in CT Variables in response to 6 months of exercise training

Control $\square \mathbf{4}$ kcal/kg/wk $\square \mathbf{8 ~ k c a l / k g / w k} \square 12$ kcal/kg/wk

(75 min/wk)

(150 min/wk)

(225 min/wk)

A

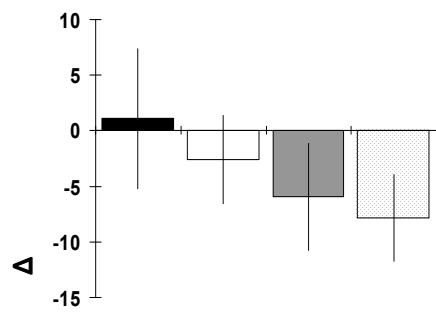

D

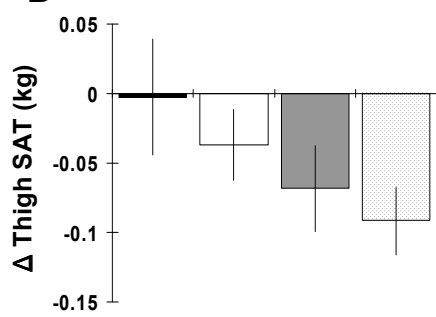

B

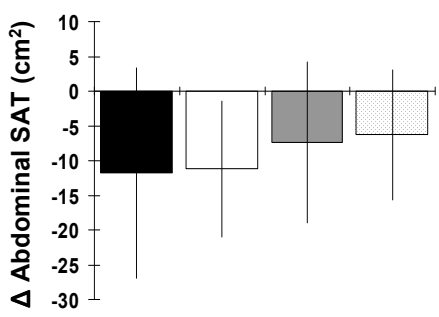

E

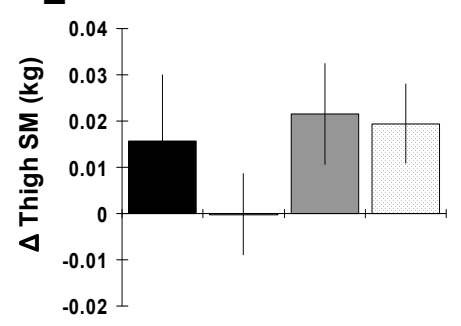

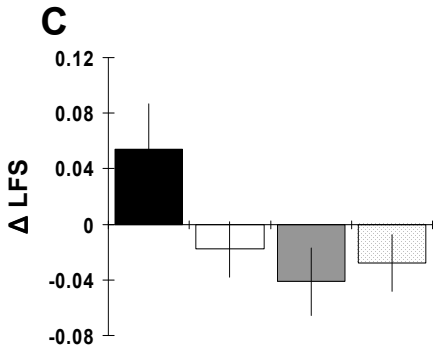

F

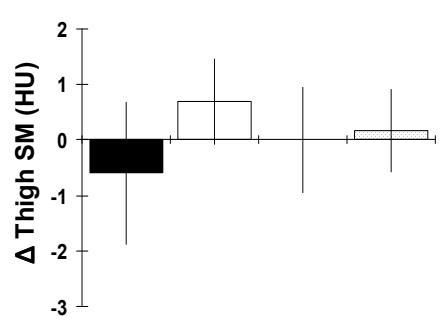




\section{Chapter 7. General Discussion}

With the rising prevalence of obesity worldwide, the proportion of people with obesity related metabolic diseases such as type 2 diabetes and cardiovascular disease has also risen. Zimmet et al. (248) estimated in 2001 that the number of individuals with type 2 diabetes will increase from 151 million in the year 2000 to 221 million in the year 2010 . However, latest estimates from the International Diabetes Federation suggest that the number of individuals with type 2 diabetes has already reached 246 million this year (249). The increases in the prevalence of obesity related diseases have resulted in large economic consequences as well. In 2001, the direct and indirect costs of obesity were estimated to be $\sim 4.3$ billion dollars in Canada (250). In the United States obesity attributable expenditures are estimated to be a staggering 75 billion dollars annually, $\sim 6 \%$ of all adult medical expenditures (251). Clearly, this is a very serious problem that must be addressed by developing clinical tools that can better identify individuals at risk for obesity-related metabolic disease, and treatment strategies designed at improving metabolic risk or disease outcomes.

It is clear that abdominal obesity, as assessed by waist circumference, is a strong predictor of morbidity (5-7) and mortality (8). The association between waist circumference and health risk may be explained by its' corresponding association with abdominal subcutaneous and/or visceral AT. However, factors that influence the association between waist circumference and visceral adiposity may limit the ability of waist circumference to discern differences in health risk. For example, age-related changes in abdominal fat distribution are associated with increases in visceral AT that may not be apparent through measures of waist circumference. As clinical measures of 
visceral AT are not readily available, future research should examine whether these changes in the association between waist circumference and visceral AT influence the interpretation of health risk for a given waist circumference, and accordingly, the need for the development of age-specific waist circumference guidelines. There is also no accepted measurement landmark for measuring waist circumference, despite the substantial evidence reinforcing the importance of measuring waist circumference in predicting health risk. Currently, Health Canada (252) and the World Health Organization (253) advocate the measurement of waist circumference at the midpoint between the last rib and the iliac crest, whereas the National Institutes of Health (NIH) in the United States recommend using the top of the iliac crest (254). There are substantial differences in the absolute waist circumference value obtained at different landmarks in women $(\sim 4.5 \mathrm{~cm})$, and to a lesser degree in men $(\sim 1.0 \mathrm{~cm})(197)$. It is unclear how differences in measurement landmark impact the optimal waist circumference cut-off values that should be used to delineate increased risk. Although measures of waist circumferences at different landmarks tend to be highly correlated (197), there is evidence to suggest that as with visceral AT, the landmark used for measuring waist circumference may also influence its association with health risk $(255,256)$.

In addition to ambiguity as to the optimal landmark, the optimal method for incorporating waist circumference into clinical practice is also unclear. It is suggested that not all individuals with an elevated waist circumference are at increased health risk $(257,258)$. Indeed, Katzmarzyk et al. (257) report that individuals who present with a waist circumference greater than the NIH cut-offs (Men: $102 \mathrm{~cm}$, Women: $88 \mathrm{~cm}$ ) but have less than two metabolic risk factors for the metabolic syndrome, are not at increased 
risk for all-cause or CVD mortality. However, only $6 \%$ of men and $9 \%$ of women who are abdominally obese, are also free of other traditional metabolic risk factors (258). Currently, the optimal usage of waist circumference alone or in conjunction with other clinical measures is unclear. It is suggested that waist circumference in conjunction with fasting triglycerides may provide a simple, but clinically useful tool for the identification of individuals at risk for metabolic consequences (259). However, much of this work has been conducted in Caucasian populations, and whether these cut-off values and associations with health risk remain valid in non-Caucasian populations is less known. Clearly more work is needed to refine and clarify how waist circumference should be used in clinical practice.

Despite the large body of evidence demonstrating the deleterious effects of abdominal obesity, this notion is largely unknown to the general public (260). Moreover, the measurement of waist circumference is infrequent in primary care $(260,261)$. Recent evidence from the Shape of the Nations Survey suggests that from 2005 to 2006, there has been a dramatic 50\% increase in the number of physicians measuring waist in clinical practice (260). However, although $81 \%$ of physicians reporting that they measure waist circumference, only a third of at risk patients report having their waist circumference measured by their physician (262). These numbers are more in line with the data reported by Carson (261) who reports that less than a quarter of medical students measure waist circumference despite being educated about the importance of measuring waist, and being given a measuring tape. Clearly, much work needs to be done in order to translate scientific knowledge into medical practice. 
As with waist circumference, visceral AT is currently measured using various protocols at various landmarks. Although L4-L5 is the most commonly used landmark for assessing visceral adiposity, the findings from this thesis and other recent evidence (263) demonstrate that L4-L5 is unlikely to be the most optimal landmark for assessing the association between visceral AT and metabolic risk. Further, it appears that the optimal landmark for assessing visceral AT and related health risk may differ between men and women (263). Future research is still needed to determine whether racial differences also exist, and whether these differences are large enough to warrant alterations in the measurement protocol used for the assessment of visceral AT in clinical trials. Despite the limitations associated with assessing visceral AT at L4-L5, the majority of studies that have measured visceral AT at L4-L5 report that visceral AT is an independent correlate of health risk. Nevertheless, it is plausible that the true importance of visceral adiposity has currently been diminished to measurement landmark, as not all studies report a significant association between visceral AT and health risk $(20,93)$. Further, it is as of yet unclear whether visceral AT is a predictor of morbidity and mortality independent of traditional or other emerging risk factors $(19,20,65)$.

The final study in this thesis examined the association between exercise dose and reductions in abdominal obesity. It is established that modest doses of physical activity (ie. 30 minutes on all or most days of the week (15)) is sufficient for attaining health benefits. Further, numerous studies have reported that exercise is associated with a reduction in waist circumference and visceral AT (10-14). In spite of the wealth of literature pointing to the importance of both physical inactivity and abdominal obesity in predicting health risk, little is known about the inter-relationship between increases in 
physical activity and reductions in abdominal obesity. The findings from this thesis provides some of the first evidence that even very modest amounts of physical activity below the current guidelines for health is associated with improvements in abdominal obesity. However, exercise doses at, or $50 \%$ above the recommendations are not associated with further reductions in waist circumference. Further, these exercise doses were not associated with changes in body weight, and thus reinforce the importance of regular physical activity as a treatment strategy for abdominal obesity, and the importance of acquiring measures of waist circumference when examining strategies targeting obesity reduction. These findings also should be particularly encouraging for individuals who are resistant to weight loss, as reductions in waist circumference may provide additional motivation and positive reinforcement. Although exercise is an efficacious treatment for obesity reduction, many studies show a high recidivism among those who engage in physical activity to reduce obesity (264). Data from the National Weight Registry reinforce the notion that physical activity needs to be an integral part in the lifestyle of previously obese individuals (265). However, the question of the exercise dose required in order to maintain or induce obesity reduction, and the differences in the interventions required for these stages of change is largely unknown and requires further investigation. 


\section{Summary and Conclusions}

In conclusion, abdominal obesity and physical inactivity are both associated with deleterious health outcomes. Given the high prevalence of each, attention should be given to developing therapeutic interventions targeted at reducing these risk factors. As even modest amounts of physical activity are associated with reductions in abdominal obesity and improvements in health risk, physical activity should be included in therapeutic interventions directed at treating obesity and obesity-related metabolic disorders. However, it is clear that there is much work yet to be done to clarify the optimal cut-offs, the optimal measurement landmarks and how waist circumference should be used in clinical practice. Moreover, it is important to examine ways to convince health practitioners, and educate the general population on the importance of

measuring waist circumference, and preventing abdominal obesity and related morbidity. 


\section{References}

1. Okosun IS, Chandra KMD, Boev A, Boltri JM, Choi ST, Parish DC and Dever GEA: Abdominal adiposity in U.S. adults: prevalence and trends, 1960-2000. Preventive Medicine 39(1):197-206, 2004

2. Visscher TL and Seidell JC: Time trends (1993-1997) and seasonal variation in body mass index and waist circumference in the Netherlands. Int J Obes Relat Metab Disord 28(10):1309-16, 2004

3. Liese AD, Doring A, Hense HW and Keil U: Five year changes in waist circumference, body mass index and obesity in Augsburg, Germany. Eur J Nutr 40(6):282-8, 2001

4. Eiben G, Dey DK, Rothenberg E, Steen B, Bjorkelund C, Bengtsson C and Lissner L: Obesity in 70-year-old Swedes: secular changes over 30 years. Int J Obes (Lond) 29(7):810-7, 2005

5. Janssen I, Katzmarzyk PT and Ross R: Waist circumference and not body mass index explains obesity-related health risk. Am J Clin Nutr 79(3):379-84, 2004

6. Yusuf S, Hawken S, Ounpuu S, Bautista L, Franzosi MG, Commerford P, Lang CC, Rumboldt Z, Onen CL, Lisheng L, Tanomsup S, Wangai P, Jr., Razak F, Sharma AM and Anand SS: Obesity and the risk of myocardial infarction in 27,000 participants from 52 countries: a case-control study. Lancet 366(9497):1640-9, 2005

7. Ohlson LO, Larsson B, Svardsudd K, Welin L, Eriksson H, Wilhelmsen L, Bjorntorp $\mathrm{P}$ and Tibblin G: The influence of body fat distribution on the incidence of diabetes mellitus. 13.5 years of follow-up of the participants in the study of men born in 1913 . Diabetes 34(10):1055-8, 1985

8. Bigaard J, Tjonneland A, Thomsen BL, Overvad K, Heitmann BL and Sorensen TI: Waist circumference, BMI, smoking, and mortality in middle-aged men and women. Obes Res 11(7):895-903, 2003

9. Despres JP, Moorjani S, Lupien PJ, Tremblay A, Nadeau A and Bouchard C: Regional distribution of body fat, plasma lipoproteins, and cardiovascular disease. Arteriosclerosis 10(4):497-511, 1990

10. Ross R and Janssen I: Is abdominal fat preferentially reduced in response to exercise-induced weight loss? Med Sci Sports Exerc 31(11 Suppl):S568-72, 1999 
11. Ross R, Janssen I, Dawson J, Kungl AM, Kuk JL, Wong SL, Nguyen-Duy TB, Lee S, Kilpatrick K and Hudson R: Exercise-induced reduction in obesity and insulin resistance in women: a randomized controlled trial. Obes Res 12(5):789-98, 2004

12. Ross R, Dagnone D, Jones PJ, Smith H, Paddags A, Hudson R and Janssen I: Reduction in obesity and related comorbid conditions after diet-induced weight loss or exercise-induced weight loss in men. A randomized, controlled trial. Ann Intern Med 133(2):92-103, 2000

13. Pare A, Dumont M, Lemieux I, Brochu M, Almeras N, Lemieux S, Prud'homme D and Despres JP: Is the relationship between adipose tissue and waist girth altered by weight loss in obese men? Obes Res 9(9):526-34, 2001

14. Mayo MJ, Grantham JR and Balasekaran G: Exercise-induced weight loss preferentially reduces abdominal fat. Med Sci Sports Exerc 35(2):207-13, 2003

15. Physical activity and health: a report of the Surgeon General. 1996

16. Henriksson KM, Lindblad U, Agren B, Nilsson-Ehle P and Rastam L: Associations between body height, body composition and cholesterol levels in middle-aged men. the coronary risk factor study in southern Sweden (CRISS). Eur J Epidemiol 17(6):521-6, 2001

17. Calle EE, Thun MJ, Petrelli JM, Rodriguez C and Heath CW, Jr.: Body-mass index and mortality in a prospective cohort of U.S. adults. N Engl J Med 341(15):1097-105, 1999

18. Flegal KM, Carroll MD, Ogden CL and Johnson CL: Prevalence and trends in obesity among US adults, 1999-2000. JAMA 288(14):1723-7, 2002

19. Hayashi T, Boyko EJ, Leonetti DL, McNeely MJ, Newell-Morris L, Kahn SE and Fujimoto WY: Visceral adiposity and the prevalence of hypertension in Japanese Americans. Circulation 108(14):1718-23, 2003

20. Nicklas BJ, Penninx BW, Cesari M, Kritchevsky SB, Newman AB, Kanaya AM, Pahor M, Jingzhong D and Harris TB: Association of visceral adipose tissue with incident myocardial infarction in older men and women: the health, aging and body composition study. Am J Epidemiol 160(8):741-9, 2004

21. Kelley DE, McKolanis TM, Hegazi RA, Kuller LH and Kalhan SC: Fatty liver in type 2 diabetes mellitus: relation to regional adiposity, fatty acids, and insulin resistance. Am J Physiol Endocrinol Metab 285(4):E906-16, 2003 
22. Farin HM, Abbasi F and Reaven GM: Body mass index and waist circumference both contribute to differences in insulin-mediated glucose disposal in nondiabetic adults. Am J Clin Nutr 83(1):47-51, 2006

23. Wei M, Kampert JB, Barlow CE, Nichaman MZ, Gibbons LW, Paffenbarger RS, Jr. and Blair SN: Relationship between low cardiorespiratory fitness and mortality in normal-weight, overweight, and obese men. JAMA 282(16):1547-53, 1999

24. Kay SJ and Fiatarone Singh MA: The influence of physical activity on abdominal fat: a systematic review of the literature. Obesity Reviews 7(2):183-200, 2006

25. Zhu S, Heshka S, Wang Z, Shen W, Allison DB, Ross R and Heymsfield SB: Combination of BMI and Waist Circumference for Identifying Cardiovascular Risk Factors in Whites. Obes Res 12(4):633-45, 2004

26. Huang KC, Lee MS, Lee SD, Chang YH, Lin YC, Tu SH and Pan WH: Obesity in the elderly and its relationship with cardiovascular risk factors in Taiwan. Obes Res 13(1):170-8, 2005

27. Dalton M, Cameron AJ, Zimmet PZ, Shaw JE, Jolley D, Dunstan DW and Welborn TA: Waist circumference, waist-hip ratio and body mass index and their correlation with cardiovascular disease risk factors in Australian adults. J Intern Med 254(6):555-63, 2003

28. Wildman RP, Gu D, Reynolds K, Duan X, Wu X and He J: Are waist circumference and body mass index independently associated with cardiovascular disease risk in Chinese adults? Am J Clin Nutr 82(6):1195-202, 2005

29. Janssen I, Heymsfield SB, Allison DB, Kotler DP and Ross R: Body mass index and waist circumference independently contribute to the prediction of nonabdominal, abdominal subcutaneous, and visceral fat. Am J Clin Nutr 75(4):683-8, 2002

30. Despres JP, Prud'homme D, Pouliot MC, Tremblay A and Bouchard C: Estimation of deep abdominal adipose-tissue accumulation from simple anthropometric measurements in men. Am J Clin Nutr 54(3):471-7, 1991

31. Han TS, McNeill G, Seidell JC and Lean ME: Predicting intra-abdominal fatness from anthropometric measures: the influence of stature. Int J Obes Relat Metab Disord 21(7):587-93, 1997

32. Ross R, Shaw KD, Rissanen J, Martel Y, de Guise J and Avruch L: Sex differences in lean and adipose tissue distribution by magnetic resonance imaging: anthropometric relationships. Am J Clin Nutr 59(6):1277-85, 1994 
33. Ross R, Leger L, Morris D, de Guise J and Guardo R: Quantification of adipose tissue by MRI: relationship with anthropometric variables. $J$ Appl Physiol 72(2):787-95, 1992

34. Seidell JC, Bjorntorp P, Sjostrom L, Sannerstedt R, Krotkiewski M and Kvist H: Regional distribution of muscle and fat mass in men--new insight into the risk of abdominal obesity using computed tomography. Int J Obes 13(3):289-303, 1989

35. van der Kooy K, Leenen R, Seidell JC, Deurenberg P, Droop A and Bakker CJ: Waist-hip ratio is a poor predictor of changes in visceral fat. Am J Clin Nutr 57(3):327-33, 1993

36. Carr DB, Utzschneider KM, Hull RL, Kodama K, Retzlaff BM, Brunzell JD, Shofer JB, Fish BE, Knopp RH and Kahn SE: Intra-abdominal fat is a major determinant of the National Cholesterol Education Program Adult Treatment Panel III criteria for the metabolic syndrome. Diabetes 53(8):2087-94, 2004

37. Kuk JL, Nichaman MZ, Church TS, Blair SN and Ross R: Liver fat is not a marker of metabolic risk in lean premenopausal women. Metabolism 53(8):1066-71, 2004

38. Goodpaster BH, Krishnaswami S, Harris TB, Katsiaras A, Kritchevsky SB, Simonsick EM, Nevitt M, Holvoet P and Newman AB: Obesity, regional body fat distribution, and the metabolic syndrome in older men and women. Arch Intern Med 165(7):777-83, 2005

39. Boyko EJ, Fujimoto WY, Leonetti DL and Newell-Morris L: Visceral adiposity and risk of type 2 diabetes: a prospective study among Japanese Americans. Diabetes Care 23(4):465-71, 2000

40. Lara-Castro C, Weinsier RL, Hunter GR and Desmond R: Visceral adipose tissue in women: longitudinal study of the effects of fat gain, time, and race. Obes Res 10(9):86874,2002

41. Schreiner PJ, Terry JG, Evans GW, Hinson WH, Crouse JR, 3rd and Heiss G: Sexspecific associations of magnetic resonance imaging-derived intra-abdominal and subcutaneous fat areas with conventional anthropometric indices. The Atherosclerosis Risk in Communities Study. Am J Epidemiol 144(4):335-45, 1996

42. Park Y-W, Allison DB, Heymsfield SB and Gallagher D: Larger Amounts of Visceral Adipose Tissue in Asian Americans. Obesity Res 9(7):381-387, 2001 
43. Hoffman DJ, Wang Z, Gallagher D and Heymsfield SB: Comparison of Visceral Adipose Tissue Mass in Adult African Americans and Whites. Obesity Res 13(1):66-74, 2005

44. Kadowaki T, Sekikawa A, Murata K, Maegawa H, Takamiya T, Okamura T, ElSaed A, Miyamatsu N, Edmundowicz D, Kita Y, Sutton-Tyrrell K, Kuller LH and Ueshima H: Japanese men have larger areas of visceral adipose tissue than Caucasian men in the same levels of waist circumference in a population-based study. Int J Obes Relat Metab Disord 30(7):1163-1165, 2006

45. Wong SL, Katzmarzyk P, Nichaman MZ, Church TS, Blair SN and Ross R: Cardiorespiratory fitness is associated with lower abdominal fat independent of body mass index. Med Sci Sports Exerc 36(2):286-91, 2004

46. Janssen I, Katzmarzyk PT, Ross R, Leon AS, Skinner JS, Rao DC, Wilmore JH, Rankinen $\mathrm{T}$ and Bouchard C: Fitness alters the associations of BMI and waist circumference with total and abdominal fat. Obes Res 12(3):525-37, 2004

47. Lean ME, Han TS and Morrison CE: Waist circumference as a measure for indicating need for weight management. Bmj 311(6998):158-61, 1995

48. Zhu S, Heymsfield SB, Toyoshima H, Wang Z, Pietrobelli A and Heshka S: Raceethnicity-specific waist circumference cutoffs for identifying cardiovascular disease risk factors. Am J Clin Nutr 81(2):409-15, 2005

49. Lemieux S, Prud'homme D, Nadeau A, Tremblay A, Bouchard C and Despres JP: Seven-year changes in body fat and visceral adipose tissue in women. Association with indexes of plasma glucose-insulin homeostasis. Diabetes Care 19(9):983-91, 1996

50. Zamboni M, Armellini F, Milani MP, De Marchi M, Todesco T, Robbi R, BergamoAndreis IA and Bosello O: Body fat distribution in pre- and post-menopausal women: metabolic and anthropometric variables and their inter-relationships. Int J Obes Relat Metab Disord 16(7):495-504, 1992

51. Pouliot MC, Despres JP, Lemieux S, Moorjani S, Bouchard C, Tremblay A, Nadeau A and Lupien PJ: Waist circumference and abdominal sagittal diameter: best simple anthropometric indexes of abdominal visceral adipose tissue accumulation and related cardiovascular risk in men and women. Am J Cardiol 73(7):460-8, 1994

52. Kelley DE, Thaete FL, Troost F, Huwe T and Goodpaster BH: Subdivisions of subcutaneous abdominal adipose tissue and insulin resistance. Am J Physiol Endocrinol Metab 278(5):E941-8, 2000 
53. Lovejoy JC, Smith SR and Rood JC: Comparison of regional fat distribution and health risk factors in middle-aged white and African American women: The Healthy Transitions Study. Obes Res 9(1):10-6, 2001

54. Nguyen-Duy TB, Nichaman MZ, Church TS, Blair SN and Ross R: Visceral fat and liver fat are independent predictors of metabolic risk factors in men. Am J Physiol Endocrinol Metab 284(6):E1065-71, 2003

55. Ross R, Aru J, Freeman J, Hudson R and Janssen I: Abdominal adiposity and insulin resistance in obese men. Am J Physiol Endocrinol Metab 282(3):E657-63, 2002

56. Smith SR, Lovejoy JC, Greenway F, Ryan D, deJonge L, de la Bretonne J, Volafova $\mathrm{J}$ and Bray GA: Contributions of total body fat, abdominal subcutaneous adipose tissue compartments, and visceral adipose tissue to the metabolic complications of obesity. Metabolism 50(4):425-35, 2001

57. Despres JP, Nadeau A, Tremblay A, Ferland M, Moorjani S, Lupien PJ, Theriault G, Pinault S and Bouchard C: Role of deep abdominal fat in the association between regional adipose tissue distribution and glucose tolerance in obese women. Diabetes 38(3):304-9, 1989

58. Motoshima H, Wu X, Sinha MK, Hardy VE, Rosato EL, Barbot DJ, Rosato FE and Goldstein BJ: Differential regulation of adiponectin secretion from cultured human omental and subcutaneous adipocytes: effects of insulin and rosiglitazone. J Clin Endocrinol Metab 87(12):5662-7, 2002

59. McConnaughey MM, Sheets KA, Davis J, Privette J, Hickner R, Christian B and Barakat H: Differences in beta-adrenergic receptor densities in omental and subcutaneous adipose tissue from obese African American and Caucasian women. Metabolism 53(2):247-51, 2004

60. van Harmelen V, Dicker A, Ryden M, Hauner H, Lonnqvist F, Naslund E and Arner $\mathrm{P}$ : Increased lipolysis and decreased leptin production by human omental as compared with subcutaneous preadipocytes. Diabetes 51(7):2029-36, 2002

61. Mittelman SD, Van Citters GW, Kirkman EL and Bergman RN: Extreme insulin resistance of the central adipose depot in vivo. Diabetes 51(3):755-61, 2002

62. Bjorntorp P: "Portal" adipose tissue as a generator of risk factors for cardiovascular disease and diabetes. Arteriosclerosis 10(4):493-6, 1990

63. Matsuzawa Y: The metabolic syndrome and adipocytokines. FEBS Letters Istanbul Special Issue 580(12):2917-2921, 2006 
64. Robinson LE and Graham TE: Metabolic syndrome, a cardiovascular disease risk factor: role of adipocytokines and impact of diet and physical activity. Can J Appl

Physiol 29(6):808-29, 2004

65. Kanaya AM, Harris T, Goodpaster BH, Tylavsky F and Cummings SR:

Adipocytokines attenuate the association between visceral adiposity and diabetes in older adults. Diabetes Care 27(6):1375-80, 2004

66. Matsuzawa Y, Funahashi T and Nakamura T: Molecular mechanism of metabolic syndrome X: contribution of adipocytokines adipocyte-derived bioactive substances. Ann N Y Acad Sci 892:146-54, 1999

67. Fasshauer M and Paschke R: Regulation of adipocytokines and insulin resistance. Diabetologia 46(12):1594-603, 2003

68. Trayhurn P and Beattie JH: Physiological role of adipose tissue: white adipose tissue as an endocrine and secretory organ. Proc Nutr Soc 60(3):329-39, 2001

69. Mohamed-Ali V, Pinkney JH and Coppack SW: Adipose tissue as an endocrine and paracrine organ. Int J Obes Relat Metab Disord 22(12):1145-58, 1998

70. Havel PJ: Control of energy homeostasis and insulin action by adipocyte hormones: leptin, acylation stimulating protein, and adiponectin. Curr Opin Lipidol 13(1):51-9, 2002

71. Havel PJ: Update on adipocyte hormones: regulation of energy balance and carbohydrate/lipid metabolism. Diabetes 53 Suppl 1:S143-51, 2004

72. Frayn KN, Karpe F, Fielding BA, Macdonald IA and Coppack SW: Integrative physiology of human adipose tissue. Int J Obes Relat Metab Disord 27(8):875-88, 2003

73. Fruhbeck G, Gomez-Ambrosi J, Muruzabal FJ and Burrell MA: The adipocyte: a model for integration of endocrine and metabolic signaling in energy metabolism regulation. Am J Physiol Endocrinol Metab 280(6):E827-47, 2001

74. Matsuzawa Y, Shimomura I, Kihara S and Funahashi T: Importance of adipocytokines in obesity-related diseases. Horm Res 60 Suppl 3:56-9, 2003

75. Matsuzawa Y: White adipose tissue and cardiovascular disease. Best Practice \& Research Clinical Endocrinology \& Metabolism 19(4):637-647, 2005

76. Tilg H and Diehl AM: Cytokines in alcoholic and nonalcoholic steatohepatitis. $N$ Engl J Med 343(20):1467-76, 2000 
77. Vendrell J, Broch M, Vilarrasa N, Molina A, Gomez JM, Gutierrez C, Simon I, Soler J and Richart C: Resistin, adiponectin, ghrelin, leptin, and proinflammatory cytokines: relationships in obesity. Obes Res 12(6):962-71, 2004

78. Faraj M, Havel PJ, Phelis S, Blank D, Sniderman AD and Cianflone K: Plasma acylation-stimulating protein, adiponectin, leptin, and ghrelin before and after weight loss induced by gastric bypass surgery in morbidly obese subjects. J Clin Endocrinol Metab 88(4):1594-602, 2003

79. Kanaya AM, Wassel Fyr C, Vittinghoff E, Harris TB, Park SW, Goodpaster BH, Tylavsky F and Cummings SR: Adipocytokines and Incident Diabetes Mellitus in Older Adults: The Independent Effect of Plasminogen Activator Inhibitor 1. Arch Intern Med 166(3):350-356, 2006

80. Kumada M, Kihara S, Sumitsuji S, Kawamoto T, Matsumoto S, Ouchi N, Arita Y, Okamoto Y, Shimomura I, Hiraoka H, Nakamura T, Funahashi T and Matsuzawa Y: Association of hypoadiponectinemia with coronary artery disease in men. Arterioscler Thromb Vasc Biol 23(1):85-9, 2003

81. Tiikkainen M, Hakkinen AM, Korsheninnikova E, Nyman T, Makimattila S and Yki-Jarvinen H: Effects of rosiglitazone and metformin on liver fat content, hepatic insulin resistance, insulin clearance, and gene expression in adipose tissue in patients with type 2 diabetes. Diabetes 53(8):2169-76, 2004

82. Skurk T and Hauner H: Obesity and impaired fibrinolysis: role of adipose production of plasminogen activator inhibitor-1. Int J Obes Relat Metab Disord 28(11):1357-64, 2004

83. Hayase H, Nomura S, Abe T and Izawa T: Relation between fat distributions and several plasma adipocytokines after exercise training in premenopausal and postmenopausal women. J Physiol Anthropol Appl Human Sci 21(2):105-13, 2002

84. De Taeye B, Smith LH and Vaughan DE: Plasminogen activator inhibitor-1: a common denominator in obesity, diabetes and cardiovascular disease. Curr Opin Pharmacol 5(2):149-54, 2005

85. Alessi MC, Peiretti F, Morange P, Henry M, Nalbone G and Juhan-Vague I: Production of plasminogen activator inhibitor 1 by human adipose tissue: possible link between visceral fat accumulation and vascular disease. Diabetes 46(5):860-7, 1997

86. Marin P, Andersson B, Ottosson M, Olbe L, Chowdhury B, Kvist H, Holm G, Sjostrom L and Bjorntorp P: The morphology and metabolism of intraabdominal adipose tissue in men. Metabolism 41(11):1242-8, 1992 
87. Rebuffe-Scrive M, Andersson B, Olbe L and Bjorntorp P: Metabolism of adipose tissue in intraabdominal depots of nonobese men and women. Metabolism 38(5):453-8, 1989

88. Abate N, Garg A, Peshock RM, Stray-Gundersen J and Grundy SM: Relationships of generalized and regional adiposity to insulin sensitivity in men. J Clin Invest 96(1):8898,1995

89. Abate N, Garg A, Coleman R, Grundy SM and Peshock RM: Prediction of total subcutaneous abdominal, intraperitoneal, and retroperitoneal adipose tissue masses in men by a single axial magnetic resonance imaging slice. Am J Clin Nutr 65(2):403-8, 1997

90. Kvist H, Chowdhury B, Grangard U, Tylen U and Sjostrom L: Total and visceral adipose-tissue volumes derived from measurements with computed tomography in adult men and women: predictive equations. Am J Clin Nutr 48(6):1351-61, 1988

91. Shen W, Punyanitya M, Wang Z, Gallagher D, St-Onge MP, Albu J, Heymsfield SB and Heshka S: Visceral adipose tissue: relations between single-slice areas and total volume. Am J Clin Nutr 80(2):271-8, 2004

92. Han TS, Kelly IE, Walsh K, Greene RM and Lean ME: Relationship between volumes and areas from single transverse scans of intra-abdominal fat measured by magnetic resonance imaging. Int J Obes Relat Metab Disord 21(12):1161-6, 1997

93. Tiikkainen M, Tamminen M, Hakkinen AM, Bergholm R, Vehkavaara S, Halavaara J, Teramo K, Rissanen A and Yki-Jarvinen H: Liver-fat accumulation and insulin resistance in obese women with previous gestational diabetes. Obes Res 10(9):859-67, 2002

94. Seppala-Lindroos A, Vehkavaara S, Hakkinen AM, Goto T, Westerbacka J, Sovijarvi A, Halavaara J and Yki-Jarvinen H: Fat accumulation in the liver is associated with defects in insulin suppression of glucose production and serum free fatty acids independent of obesity in normal men. J Clin Endocrinol Metab 87(7):3023-8, 2002

95. Kral JG, Lundholm K, Bjorntorp P, Sjostrom L and Schersten T: Hepatic lipid metabolism in severe human obesity. Metabolism 26(9):1025-31, 1977

96. Adiels M, Taskinen MR, Packard C, Caslake MJ, Soro-Paavonen A, Westerbacka J, Vehkavaara S, Hakkinen A, Olofsson SO, Yki-Jarvinen H and Boren J: Overproduction of large VLDL particles is driven by increased liver fat content in man. Diabetologia 49(4):755-65, 2006 
97. Goto T, Onuma T, Takebe K and Kral JG: The influence of fatty liver on insulin clearance and insulin resistance in non-diabetic Japanese subjects. Int J Obes Relat Metab Disord 19(12):841-5, 1995

98. Banerji MA, Buckley MC, Chaiken RL, Gordon D, Lebovitz HE and Kral JG: Liver fat, serum triglycerides and visceral adipose tissue in insulin-sensitive and insulinresistant black men with NIDDM. Int J Obes Relat Metab Disord 19(12):846-50, 1995

99. Lewis GF, Carpentier A, Adeli K and Giacca A: Disordered fat storage and mobilization in the pathogenesis of insulin resistance and type 2 diabetes. Endocr Rev 23(2):201-29, 2002

100. Festi D, Colecchia A, Sacco T, Bondi M, Roda E and Marchesini G: Hepatic steatosis in obese patients: clinical aspects and prognostic significance. Obes Rev 5(1):2742, 2004

101. Bugianesi E, Zannoni C, Vanni E, Marzocchi R and Marchesini G: Non-alcoholic fatty liver and insulin resistance: a cause-effect relationship? Dig Liver Dis 36(3):165-73, 2004

102. Luyckx FH, Lefebvre PJ and Scheen AJ: Non-alcoholic steatohepatitis: association with obesity and insulin resistance, and influence of weight loss. Diabetes Metab 26(2):98-106, 2000

103. Marchesini G, Brizi M, Morselli-Labate AM, Bianchi G, Bugianesi E, McCullough AJ, Forlani G and Melchionda N: Association of nonalcoholic fatty liver disease with insulin resistance. Am J Med 107(5):450-5, 1999

104. Haque M and Sanyal AJ: The metabolic abnormalities associated with nonalcoholic fatty liver disease. Best Pract Res Clin Gastroenterol 16(5):709-31, 2002

105. Nielsen S, Guo Z, Johnson CM, Hensrud DD and Jensen MD: Splanchnic lipolysis in human obesity. J Clin Invest 113(11):1582-8, 2004

106. Thomas EL, Hamilton G, Patel N, O'Dwyer R, Dore CJ, Goldin RD, Bell JD and Taylor-Robinson SD: Hepatic triglyceride content and its relation to body adiposity: a magnetic resonance imaging and proton magnetic resonance spectroscopy study. Gut 54(1):122-7, 2005

107. Westerbacka J, Corner A, Tiikkainen M, Tamminen M, Vehkavaara S, Hakkinen AM, Fredriksson J and Yki-Jarvinen H: Women and men have similar amounts of liver and intra-abdominal fat, despite more subcutaneous fat in women: implications for sex differences in markers of cardiovascular risk. Diabetologia 47(8):1360-9, 2004 
108. Lemieux I: Energy partitioning in gluteal-femoral fat: does the metabolic fate of triglycerides affect coronary heart disease risk? Arterioscler Thromb Vasc Biol 24(5):795-7, 2004

109. Goodpaster BH, Kelley DE, Thaete FL, He J and Ross R: Skeletal muscle attenuation determined by computed tomography is associated with skeletal muscle lipid content. J Appl Physiol 89(1):104-10, 2000

110. Kuk JL, Katzmarzyk PT, Nichaman MZ, Church TS, Blair SN and Ross R: Visceral Fat Is an Independent Predictor of All-cause Mortality in Men. Obes Res 14(2):336-341, 2006

111. Kelley DE and Goodpaster BH: Skeletal muscle triglyceride. An aspect of regional adiposity and insulin resistance. Diabetes Care 24(5):933-41, 2001

112. Unger RH: "Weapons of lean body mass destruction: the role of ectopic lipids in the metabolic syndrome". Endocrinology, 2003

113. Petersen KF, Oral EA, Dufour S, Befroy D, Ariyan C, Yu C, Cline GW, DePaoli AM, Taylor SI, Gorden P and Shulman GI: Leptin reverses insulin resistance and hepatic steatosis in patients with severe lipodystrophy. J Clin Invest 109(10): 1345-50, 2002

114. Heilbronn L, Smith SR and Ravussin E: Failure of fat cell proliferation, mitochondrial function and fat oxidation results in ectopic fat storage, insulin resistance and type II diabetes mellitus. Int J Obes Relat Metab Disord 28 Suppl 4:S12-21, 2004

115. Gupta G, Cases JA, She L, Ma XH, Yang XM, Hu M, Wu J, Rossetti L and Barzilai N: Ability of insulin to modulate hepatic glucose production in aging rats is impaired by fat accumulation. Am J Physiol Endocrinol Metab 278(6):E985-91, 2000

116. Slawik M and Vidal-Puig AJ: Lipotoxicity, overnutrition and energy metabolism in aging. Ageing Res Rev 5(2):144-64, 2006

117. Haap M, Siewecke C, Thamer C, Machann J, Schick F, Haring HU, Szeimies RM and Stumvoll M: Multiple symmetric lipomatosis: a paradigm of metabolically innocent obesity? Diabetes Care 27(3):794-5, 2004

118. Pietilainen KH, Rissanen A, Kaprio J, Makimattila S, Hakkinen AM, Westerbacka $\mathrm{J}$, Sutinen J, Vehkavaara S and Yki-Jarvinen H: Acquired obesity is associated with increased liver fat, intra-abdominal fat, and insulin resistance in young adult monozygotic twins. Am J Physiol Endocrinol Metab 288(4):E768-74, 2005 
119. Tiikkainen M, Bergholm R, Vehkavaara S, Rissanen A, Hakkinen AM, Tamminen $\mathrm{M}$, Teramo $\mathrm{K}$ and Yki-Jarvinen $\mathrm{H}$ : Effects of identical weight loss on body composition and features of insulin resistance in obese women with high and low liver fat content. Diabetes 52(3):701-7, 2003

120. Westerbacka J, Lammi K, Hakkinen AM, Rissanen A, Salminen I, Aro A and YkiJarvinen H: Dietary fat content modifies liver fat in overweight nondiabetic subjects. $J$ Clin Endocrinol Metab 90(5):2804-9, 2005

121. Combs TP, Berg AH, Obici S, Scherer PE and Rossetti L: Endogenous glucose production is inhibited by the adipose-derived protein Acrp30. J. Clin. Invest. 108(12):1875-1881, 2001

122. Kintscher $U$ and Law RE: PPARgamma-mediated insulin sensitization: the importance of fat versus muscle. Am J Physiol Endocrinol Metab 288(2):E287-91, 2005

123. Matsui J, Terauchi Y, Kubota N, Takamoto I, Eto K, Yamashita T, Komeda K, Yamauchi T, Kamon J, Kita S, Noda M and Kadowaki T: Pioglitazone reduces islet triglyceride content and restores impaired glucose-stimulated insulin secretion in heterozygous peroxisome proliferator-activated receptor-gamma-deficient mice on a high-fat diet. Diabetes 53(11):2844-54, 2004

124. Bajaj M, Suraamornkul S, Piper P, Hardies LJ, Glass L, Cersosimo E, Pratipanawatr T, Miyazaki Y and DeFronzo RA: Decreased plasma adiponectin concentrations are closely related to hepatic fat content and hepatic insulin resistance in pioglitazone-treated type 2 diabetic patients. J Clin Endocrinol Metab 89(1):200-6, 2004

125. Kelly I, Han T, Walsh K and Lean M: Effects of a thiazolidinedione compound on body fat and fat distribution of patients with type 2 diabetes. Diabetes Care 22(2):288293, 1999

126. Mori Y, Murakawa Y, Okada K, Horikoshi H, Yokoyama J, Tajima N and Ikeda Y: Effect of troglitazone on body fat distribution in type 2 diabetic patients. Diabetes Care 22(6):908-912, 1999

127. Angulo P and Lindor KD: Non-alcoholic fatty liver disease. $J$ Gastroenterol Hepatol 17 Suppl:S186-90, 2002

128. Panicek DM, Giess CS and Schwartz LH: Qualitative assessment of liver for fatty infiltration on contrast-enhanced CT: is muscle a better standard of reference than spleen? J Comput Assist Tomogr 21(5):699-705, 1997 
129. Longo R, Ricci C, Masutti F, Vidimari R, Croce LS, Bercich L, Tiribelli C and Dalla Palma L: Fatty infiltration of the liver. Quantification by $1 \mathrm{H}$ localized magnetic resonance spectroscopy and comparison with computed tomography. Invest Radiol 28(4):297-302, 1993

130. Piekarski J, Goldberg HI, Royal SA, Axel L and Moss AA: Difference between liver and spleen $\mathrm{CT}$ numbers in the normal adult: its usefulness in predicting the presence of diffuse liver disease. Radiology 137(3):727-9, 1980

131. Laurin J: Motion - All patients with NASH need to have a liver biopsy: Arguments against the motion. Can J Gastroenterol 16(10):722-6, 2002

132. Bydder GM, Chapman RW, Harry D, Bassan L, Sherlock S and Kreel L:

Computed tomography attenuation values in fatty liver. J Comput Tomogr 5(1):33-5, 1981

133. Bydder GM, Kreel L, Chapman RW, Harry D, Sherlock S and Bassan L: Accuracy of computed tomography in diagnosis of fatty liver. $\mathrm{Br}$ Med $J$ 281(6247):1042, 1980

134. Pamilo M, Sotaniemi EA, Suramo I, Lahde S and Arranto AJ: Evaluation of liver steatotic and fibrous content by computerized tomography and ultrasound. Scand $J$ Gastroenterol 18(6):743-7, 1983

135. Longo R, Pollesello P, Ricci C, Masutti F, Kvam BJ, Bercich L, Croce LS, Grigolato P, Paoletti S, de Bernard B and et al.: Proton MR spectroscopy in quantitative in vivo determination of fat content in human liver steatosis. J Magn Reson Imaging 5(3):281-5, 1995

136. Thomsen C, Becker U, Winkler K, Christoffersen P, Jensen M and Henriksen O: Quantification of liver fat using magnetic resonance spectroscopy. Magn Reson Imaging 12(3):487-95, 1994

137. Marks SJ, Moore NR, Ryley NG, Clark ML, Pointon JJ, Strauss BJ and Hockaday TD: Measurement of liver fat by MRI and its reduction by dexfenfluramine in NIDDM. Int J Obes Relat Metab Disord 21(4):274-9, 1997

138. Saadeh S, Younossi ZM, Remer EM, Gramlich T, Ong JP, Hurley M, Mullen KD, Cooper JN and Sheridan MJ: The utility of radiological imaging in nonalcoholic fatty liver disease. Gastroenterology 123(3):745-50, 2002

139. Szczepaniak LS, Nurenberg P, Leonard D, Browning JD, Reingold JS, Grundy S, Hobbs HH and Dobbins RL: Magnetic resonance spectroscopy to measure hepatic 
triglyceride content: prevalence of hepatic steatosis in the general population. $\mathrm{Am} J$ Physiol Endocrinol Metab 288(2):E462-8, 2005

140. Ricci C, Longo R, Gioulis E, Bosco M, Pollesello P, Masutti F, Croce LS, Paoletti $\mathrm{S}$, de Bernard B, Tiribelli C and Dalla Palma L: Noninvasive in vivo quantitative assessment of fat content in human liver. J Hepatol 27(1):108-13, 1997

141. Davidson LE, Kuk JL, Church TS and Ross R: Protocol for Measurement of Liver Fat by Computed Tomography. J Appl Physiol, 2005

142. Joy D, Thava VR and Scott BB: Diagnosis of fatty liver disease: is biopsy necessary? Eur J Gastroenterol Hepatol 15(5):539-43, 2003

143. Boesch C, Decombaz J, Slotboom J and Kreis R: Observation of intramyocellular lipids by means of $1 \mathrm{H}$ magnetic resonance spectroscopy. Proc Nutr Soc 58(4):841-50, 1999

144. Boesch $\mathrm{C}$ and Kreis R: Observation of intramyocellular lipids by $1 \mathrm{H}$-magnetic resonance spectroscopy. Ann N Y Acad Sci 904:25-31, 2000

145. Szczepaniak LS, Babcock EE, Schick F, Dobbins RL, Garg A, Burns DK, McGarry JD and Stein DT: Measurement of intracellular triglyceride stores by $\mathrm{H}$ spectroscopy: validation in vivo. Am J Physiol 276(5 Pt 1):E977-89, 1999

146. Schick F, Eismann B, Jung WI, Bongers H, Bunse M and Lutz O: Comparison of localized proton NMR signals of skeletal muscle and fat tissue in vivo: two lipid compartments in muscle tissue. Magn Reson Med 29(2):158-67, 1993

147. DeFronzo RA, Gunnarsson R, Bjorkman O, Olsson M and Wahren J: Effects of insulin on peripheral and splanchnic glucose metabolism in noninsulin-dependent (type II) diabetes mellitus. $J$ Clin Invest 76(1):149-55, 1985

148. Pan DA, Lillioja S, Kriketos AD, Milner MR, Baur LA, Bogardus C, Jenkins AB and Storlien LH: Skeletal muscle triglyceride levels are inversely related to insulin action. Diabetes 46(6):983-8, 1997

149. Bachmann OP, Dahl DB, Brechtel K, Machann J, Haap M, Maier T, Loviscach M, Stumvoll M, Claussen CD, Schick F, Haring HU and Jacob S: Effects of intravenous and dietary lipid challenge on intramyocellular lipid content and the relation with insulin sensitivity in humans. Diabetes 50(11):2579-84, 2001 
150. Greco AV, Mingrone G, Giancaterini A, Manco M, Morroni M, Cinti S, Granzotto M, Vettor R, Camastra S and Ferrannini E: Insulin resistance in morbid obesity: reversal with intramyocellular fat depletion. Diabetes 51(1):144-51, 2002

151. Forouhi NG, Jenkinson G, Thomas EL, Mullick S, Mierisova S, Bhonsle U, McKeigue PM and Bell JD: Relation of triglyceride stores in skeletal muscle cells to central obesity and insulin sensitivity in European and South Asian men. Diabetologia 42(8):932-5, 1999

152. Krssak M, Falk Petersen K, Dresner A, DiPietro L, Vogel SM, Rothman DL, Roden M and Shulman GI: Intramyocellular lipid concentrations are correlated with insulin sensitivity in humans: a 1H NMR spectroscopy study. Diabetologia 42(1):113-6, 1999

153. Goodpaster BH, He J, Watkins S and Kelley DE: Skeletal muscle lipid content and insulin resistance: evidence for a paradox in endurance-trained athletes. $J$ Clin Endocrinol Metab 86(12):5755-61, 2001

154. Steidle G, Machann J, Claussen CD and Schick F: Separation of Intra- and Extramyocellular Lipid Signals in Proton MR Spectra by Determination of Their Magnetic Field Distribution. J Magn Reson 154(2):228-35, 2002

155. Hwang JH, Pan JW, Heydari S, Hetherington HP and Stein DT: Regional differences in intramyocellular lipids in humans observed by in vivo 1H-MR spectroscopic imaging. J Appl Physiol 90(4):1267-74, 2001

156. Lee CD, Blair SN and Jackson AS: Cardiorespiratory fitness, body composition, and all-cause and cardiovascular disease mortality in men. Am J Clin Nutr 69(3):373-80, 1999

157. Katzmarzyk PT, Church TS and Blair SN: Cardiorespiratory fitness attenuates the effects of the metabolic syndrome on all-cause and cardiovascular disease mortality in men. Arch Intern Med 164(10):1092-7, 2004

158. Thompson PD, Crouse SF, Goodpaster B, Kelley D, Moyna N and Pescatello L: The acute versus the chronic response to exercise. Med Sci Sports Exerc 33(6 Suppl):S438-45; discussion S452-3, 2001

159. King DS, Baldus PJ, Sharp RL, Kesl LD, Feltmeyer TL and Riddle MS: Time course for exercise-induced alterations in insulin action and glucose tolerance in middleaged people. J Appl Physiol 78(1):17-22, 1995 
160. Coudert J and Van Praagh E: Endurance exercise training in the elderly: effects on cardiovascular function. Curr Opin Clin Nutr Metab Care 3(6):479-483, 2000

161. Poehlman ET, Dvorak RV, DeNino WF, Brochu M and Ades PA: Effects of resistance training and endurance training on insulin sensitivity in nonobese, young women: a controlled randomized trial. J Clin Endocrinol Metab 85(7):2463-8, 2000

162. Short KR, Vittone JL, Bigelow ML, Proctor DN, Rizza RA, Coenen-Schimke JM and Nair KS: Impact of aerobic exercise training on age-related changes in insulin sensitivity and muscle oxidative capacity. Diabetes 52(8):1888-96, 2003

163. Lee S, Kuk JL, Davidson LE, Hudson R, Kilpatrick K, Graham TE and Ross R: Exercise without weight loss is an effective strategy for obesity reduction in obese individuals with and without Type 2 diabetes. J Appl Physiol 99(3):1220-5, 2005

164. Thomas EL, Brynes AE, McCarthy J, Goldstone AP, Hajnal JV, Saeed N, Frost G and Bell JD: Preferential loss of visceral fat following aerobic exercise, measured by magnetic resonance imaging. Lipids 35(7):769-76, 2000

165. Perseghin G, Price TB, Petersen KF, Roden M, Cline GW, Gerow K, Rothman DL and Shulman GI: Increased glucose transport-phosphorylation and muscle glycogen synthesis after exercise training in insulin-resistant subjects. N Engl J Med 335(18):135762,1996

166. Cullinane E, Siconolfi S, Saritelli A and Thompson PD: Acute decrease in serum triglycerides with exercise: is there a threshold for an exercise effect? Metabolism 31(8):844-7, 1982

167. Lee S, Kuk JL, Katzmarzyk PT, Blair SN, Church TS and Ross R:

Cardiorespiratory fitness attenuates metabolic risk independent of abdominal subcutaneous and visceral fat in men. Diabetes Care 28(4):895-901, 2005

168. Katzel LI, Bleecker ER, Colman EG, Rogus EM, Sorkin JD and Goldberg AP: Effects of weight loss vs aerobic exercise training on risk factors for coronary disease in healthy, obese, middle-aged and older men. A randomized controlled trial. JAMA 274(24):1915-21, 1995

169. Katzmarzyk PT, Leon AS, Rankinen T, Gagnon J, Skinner JS, Wilmore JH, Rao DC and Bouchard C: Changes in blood lipids consequent to aerobic exercise training related to changes in body fatness and aerobic fitness. Metabolism 50(7):841-8, 2001

170. Canada's physical activity guide to healthy active living. 1998 
171. Saris WH, Blair SN, van Baak MA, Eaton SB, Davies PS, Di Pietro L, Fogelholm M, Rissanen A, Schoeller D, Swinburn B, Tremblay A, Westerterp KR and Wyatt H:

How much physical activity is enough to prevent unhealthy weight gain? Outcome of the IASO 1st Stock Conference and consensus statement. Obes Rev 4(2):101-14, 2003

172. Blair SN and Lamonte MJ: How much and what type of physical activity is enough?: what physicians should tell their patients. Arch Intern Med 165(20):2324-5, 2005

173. Binder EF, Birge SJ and Kohrt WM: Effects of endurance exercise and hormone replacement therapy on serum lipids in older women. J Am Geriatr Soc 44(3):231-6, 1996

174. Slentz CA, Duscha BD, Johnson JL, Ketchum K, Aiken LB, Samsa GP, Houmard JA, Bales CW and Kraus WE: Effects of the amount of exercise on body weight, body composition, and measures of central obesity: STRRIDE--a randomized controlled study. Arch Intern Med 164(1):31-9, 2004

175. Irwin ML, Yasui Y, Ulrich CM, Bowen D, Rudolph RE, Schwartz RS, Yukawa M, Aiello E, Potter JD and McTiernan A: Effect of exercise on total and intra-abdominal body fat in postmenopausal women: a randomized controlled trial. JAMA 289(3):323-30, 2003

176. DiPietro L, Seeman TE, Stachenfeld NS, Katz LD and Nadel ER: Moderateintensity aerobic training improves glucose tolerance in aging independent of abdominal adiposity. J Am Geriatr Soc 46(7):875-9, 1998

177. Mourier A, Gautier JF, De Kerviler E, Bigard AX, Villette JM, Garnier JP, Duvallet A, Guezennec CY and Cathelineau G: Mobilization of visceral adipose tissue related to the improvement in insulin sensitivity in response to physical training in NIDDM. Effects of branched-chain amino acid supplements. Diabetes Care 20(3):385-91, 1997

178. Hellenius ML, de Faire U, Berglund B, Hamsten A and Krakau I: Diet and exercise are equally effective in reducing risk for cardiovascular disease. Results of a randomized controlled study in men with slightly to moderately raised cardiovascular risk factors. Atherosclerosis 103(1):81-91, 1993

179. O'Donovan G, Owen A, Bird SR, Kearney EM, Nevill AM, Jones DW and WoolfMay K: Changes in cardiorespiratory fitness and coronary heart disease risk factors following 24 wk of moderate- or high-intensity exercise of equal energy cost. $J \mathrm{Appl}$ Physiol 98(5):1619-25, 2005 
180. Slentz CA, Aiken LB, Houmard JA, Bales CW, Johnson JL, Tanner CJ, Duscha BD and Kraus WE: Inactivity, exercise, and visceral fat. STRRIDE: a randomized, controlled study of exercise intensity and amount. J Appl Physiol 99(4):1613-8, 2005

181. Kraus WE, Houmard JA, Duscha BD, Knetzger KJ, Wharton MB, McCartney JS, Bales CW, Henes S, Samsa GP, Otvos JD, Kulkarni KR and Slentz CA: Effects of the Amount and Intensity of Exercise on Plasma Lipoproteins. N Engl J Med 347(19):14831492,2002

182. Delecluse C, Colman V, Roelants M, Verschueren S, Derave W, Ceux T, Eijnde BO, Seghers J, Pardaens K and Brumagne S: Exercise programs for older men: mode and intensity to induce the highest possible health-related benefits. Preventive Medicine 39(4):823-833, 2004

183. Murtagh EM, Boreham CAG, Nevill A, Hare LG and Murphy MH: The effects of 60 minutes of brisk walking per week, accumulated in two different patterns, on cardiovascular risk. Preventive Medicine 41(1):92-97, 2005

184. Potteiger JA, Jacobsen DJ, Donnelly JE and Hill JO: Glucose and insulin responses following 16 months of exercise training in overweight adults: the Midwest Exercise Trial. Metabolism 52(9):1175-81, 2003

185. Shojaee-Moradie F, Baynes KC, Pentecost C, Bell JD, Thomas EL, Jackson NC, Stolinski M, Whyte M, Lovell D, Bowes SB, Gibney J, Jones RH and Umpleby AM: Exercise training reduces fatty acid availability and improves the insulin sensitivity of glucose metabolism. Diabetologia 50(2):404-413, 2007

186. Boudou P, Sobngwi E, Mauvais-Jarvis F, Vexiau P and Gautier JF: Absence of exercise-induced variations in adiponectin levels despite decreased abdominal adiposity and improved insulin sensitivity in type 2 diabetic men. Eur J Endocrinol 149(5):421-4, 2003

187. Hays NP, Starling RD, Sullivan DH, Fluckey JD, Coker RH and Evans WJ: Comparison of insulin sensitivity assessment indices with euglycemic-hyperinsulinemic clamp data after a dietary and exercise intervention in older adults. Metabolism 55(4):525-532, 2006

188. Park SK, Park JH, Kwon YC, Kim HS, Yoon MS and Park HT: The effect of combined aerobic and resistance exercise training on abdominal fat in obese middle-aged women. J Physiol Anthropol Appl Human Sci 22(3):129-35, 2003

189. Cuff DJ, Meneilly GS, Martin A, Ignaszewski A, Tildesley HD and Frohlich JJ: Effective exercise modality to reduce insulin resistance in women with type 2 diabetes. Diabetes Care 26(11):2977-82, 2003 
190. Donnelly JE, Hill JO, Jacobsen DJ, Potteiger J, Sullivan DK, Johnson SL, Heelan K, Hise M, Fennessey PV, Sonko B, Sharp T, Jakicic JM, Blair SN, Tran ZV, Mayo M, Gibson C and Washburn RA: Effects of a 16-month randomized controlled exercise trial on body weight and composition in young, overweight men and women: the Midwest Exercise Trial. Arch Intern Med 163(11):1343-50, 2003

191. Stewart KJ, Bacher AC, Turner K, Lim JG, Hees PS, Shapiro EP, Tayback M and Ouyang P: Exercise and risk factors associated with metabolic syndrome in older adults. Am J Prev Med 28(1):9-18, 2005

192. Kotani K, Tokunaga K, Fujioka S, Kobatake T, Keno Y, Yoshida S, Shimomura I, Tarui S and Matsuzawa Y: Sexual dimorphism of age-related changes in whole-body fat distribution in the obese. Int J Obes Relat Metab Disord 18(4):207-2, 1994

193. DeNino WF, Tchernof A, Dionne IJ, Toth MJ, Ades PA, Sites CK and Poehlman ET: Contribution of abdominal adiposity to age-related differences in insulin sensitivity and plasma lipids in healthy nonobese women. Diabetes Care 24(5):925-32, 2001

194. Ross R, Rissanen J, Pedwell H, Clifford J and Shragge P: Influence of diet and exercise on skeletal muscle and visceral adipose tissue in men. $J$ Appl Physiol 81(6):2445-55, 1996

195. Mitsiopoulos N, Baumgartner RN, Heymsfield SB, Lyons W, Gallagher D and Ross R: Cadaver validation of skeletal muscle measurement by magnetic resonance imaging and computerized tomography. J Appl Physiol 85(1):115-22, 1998

196. Snyder WS, Cooke MJ, Manssett ES, Larhansen LT, Howells GP and Tipton IH: Report of the Task Group on Reference Man. Eds. Oxford, Pergamon, 1975

197. Wang J, Thornton JC, Bari S, Williamson B, Gallagher D, Heymsfield SB, Horlick M, Kotler D, Laferrere B, Mayer L, Pi-Sunyer FX and Pierson RN, Jr.: Comparisons of waist circumferences measured at 4 sites. Am J Clin Nutr 77(2):379-84, 2003

198. Han TS, Seidell JC, Currall JE, Morrison CE, Deurenberg P and Lean ME: The influences of height and age on waist circumference as an index of adiposity in adults. Int J Obes Relat Metab Disord 21(1):83-9, 1997

199. Toth MJ, Tchernof A, Sites CK and Poehlman ET: Effect of menopausal status on body composition and abdominal fat distribution. Int J Obes Relat Metab Disord 24(2):226-31, 2000 
200. Guthrie JR, Dennerstein L, Taffe JR, Ebeling PR, Randolph JF, Burger HG and Wark JD: Central abdominal fat and endogenous hormones during the menopausal transition. Fertil Steril 79(6):1335-40, 2003

201. Sumino H, Ichikawa S, Yoshida A, Murakami M, Kanda T, Mizunuma H, Sakamaki T and Kurabayashi M: Effects of hormone replacement therapy on weight, abdominal fat distribution, and lipid levels in Japanese postmenopausal women. Int $J$ Obes Relat Metab Disord 27(9):1044-51, 2003

202. Tchernof A, Desmeules A, Richard C, Laberge P, Daris M, Mailloux J, Rheaume C and Dupont P: Ovarian hormone status and abdominal visceral adipose tissue metabolism. J Clin Endocrinol Metab 89(7):3425-30, 2004

203. Han TS, van Leer EM, Seidell JC and Lean ME: Waist circumference action levels in the identification of cardiovascular risk factors: prevalence study in a random sample. Bmj 311(7017):1401-5, 1995

204. Janssen I, Katzmarzyk PT and Ross R: Body mass index, waist circumference, and health risk: evidence in support of current National Institutes of Health guidelines. Arch Intern Med 162(18):2074-9, 2002

205. Executive summary of the clinical guidelines on the identification, evaluation, and treatment of overweight and obesity in adults. Arch Intern Med 158(17):1855-67, 1998

206. Pascot A, Lemieux S, Lemieux I, Prud'homme D, Tremblay A, Bouchard C, Nadeau A, Couillard C, Tchernof A, Bergeron J and Despres JP: Age-related increase in visceral adipose tissue and body fat and the metabolic risk profile of premenopausal women. Diabetes Care 22(9):1471-8, 1999

207. Hill JO, Sidney S, Lewis CE, Tolan K, Scherzinger AL and Stamm ER: Racial differences in amounts of visceral adipose tissue in young adults: the CARDIA (Coronary Artery Risk Development in Young Adults) study. Am J Clin Nutr 69(3):381-7, 1999

208. Ford ES: Risks for all-cause mortality, cardiovascular disease, and diabetes associated with the metabolic syndrome: a summary of the evidence. Diabetes Care 28(7):1769-78, 2005

209. Rebuffe-Scrive M, Anderson B, Olbe L and Bjorntorp P: Metabolism of adipose tissue in intraabdominal depots in severely obese men and women. Metabolism 39(10):1021-5, 1990

210. Eckel RH, Grundy SM and Zimmet PZ: The metabolic syndrome. Lancet 365(9468):1415-28, 2005 
211. Executive Summary of The Third Report of The National Cholesterol Education Program (NCEP) Expert Panel on Detection, Evaluation, And Treatment of High Blood Cholesterol In Adults (Adult Treatment Panel III). JAMA 285(19):2486-97, 2001

212. The IDF consensus worldwide definition of the metabolic syndrome [article online], 2005. Available from http://www.idf.org/webdata/docs/IDF_Metasyndrome definition.pdf. Accessed 13 July 2000

213. Rich S and McLaughlin VV: Detection of subclinical cardiovascular disease: the emerging role of electron beam computed tomography. Prev Med 34(1):1-10, 2002

214. Lee S, Janssen I and Ross R: Interindividual variation in abdominal subcutaneous and visceral adipose tissue: influence of measurement site. $J$ Appl Physiol 97(3):948-54, 2004

215. Bo WJ, Wolfman NT, Krueger WA, Carr JJ, Bowden RL and Meschan I: Basic Atlas of Sectional Anatomy with Correlated Imaging. 3rd Edition. Bralow L, Eds. Philadelphia, Saunders Company, 1998

216. Hotelling $\mathrm{H}$ : The selection of variants for use in prediction with some comments on the general problem of nuisance parameters. Ann Math Stat 11:271-283, 1940

217. Bradley J: Distribution-free statistical tests. Eds. London, Prentice-Hall, 1968

218. Bower JF, Vadlamudi S and Barakat HA: Ethnic differences in in vitro glyceride synthesis in subcutaneous and omental adipose tissue. Am J Physiol Endocrinol Metab 283(5):E988-93, 2002

219. Okosun IS, Liao Y, Rotimi CN, Prewitt TE and Cooper RS: Abdominal adiposity and clustering of multiple metabolic syndrome in White, Black and Hispanic americans. Ann Epidemiol 10(5):263-70, 2000

220. Despres JP, Lemieux S, Lamarche B, Prud'homme D, Moorjani S, Brun LD, Gagne $\mathrm{C}$ and Lupien PJ: The insulin resistance-dyslipidemic syndrome: contribution of visceral obesity and therapeutic implications. Int J Obes Relat Metab Disord 19 Suppl 1:S76-86, 1995

221. Prentice RL and Pyke R: Logistic disease incidence models and case-control studies. Biometrika 66:403-411, 1979 
222. Despres JP and Lamarche B: Effects of diet and physical activity on adiposity and body fat distribution: implications for the prevention of cardiovascular disease. Nutrition Research Reviews 6:137-159, 1993

223. Scheen AJ and Luyckx FH: Obesity and liver disease. Best Pract Res Clin Endocrinol Metab 16(4):703-16, 2002

224. Yki-Jarvinen $\mathrm{H}$ and Westerbacka J: The fatty liver and insulin resistance. Curr Mol Med 5(3):287-95, 2005

225. Wajchenberg BL: Subcutaneous and visceral adipose tissue: their relation to the metabolic syndrome. Endocr Rev 21(6):697-738, 2000

226. Hickman IJ, Jonsson JR, Prins JB, Ash S, Purdie DM, Clouston AD and Powell EE: Modest weight loss and physical activity in overweight patients with chronic liver disease results in sustained improvements in alanine aminotransferase, fasting insulin, and quality of life. Gut 53(3):413-9, 2004

227. Janssen I and Ross R: Effects of sex on the change in visceral, subcutaneous adipose tissue and skeletal muscle in response to weight loss. Int J Obes Relat Metab Disord 23(10):1035-46, 1999

228. Ross R, Rissanen J and Hudson R: Sensitivity associated with the identification of visceral adipose tissue levels using waist circumference in men and women: effects of weight loss. Int J Obes Relat Metab Disord 20(6):533-8, 1996

229. Blair SN, Goodyear NN, Gibbons LW and Cooper KH: Physical fitness and incidence of hypertension in healthy normotensive men and women. JAMA 252(4):48790, 1984

230. Bigaard J, Frederiksen K, Tjonneland A, Thomsen BL, Overvad K, Heitmann BL and Sorensen TI: Waist circumference and body composition in relation to all-cause mortality in middle-aged men and women. Int J Obes Relat Metab Disord 29(7):778-84, 2005

231. Katzmarzyk PT and Craig CL: Independent effects of waist circumference and physical activity on all-cause mortality in Canadian women. Appl Physiol Nutr Metab 31(3):271-6, 2006

232. Morss GM, Jordan AN, Skinner JS, Dunn AL, Church TS, Earnest CP, Kampert JB, Jurca R and Blair SN: Dose Response to Exercise in Women aged 45-75 yr (DREW): design and rationale. Med Sci Sports Exerc 36(2):336-44, 2004 
233. Kvist $\mathrm{H}$, Sjostrom $\mathrm{L}$ and Tylen $\mathrm{U}$ : Adipose tissue volume determinations in women by computed tomography: technical considerations. Int J Obes 10(1):53-67, 1986

234. Davidson LE, Kuk JL, Church TS and Ross R: Protocol for measurement of liver fat by computed tomography. J Appl Physiol 100(3):864-8, 2006

235. Goodpaster BH, Thaete FL and Kelley DE: Thigh adipose tissue distribution is associated with insulin resistance in obesity and in type 2 diabetes mellitus. Am J Clin Nutr 71(4):885-92, 2000

236. Goodpaster BH, Thaete FL, Simoneau JA and Kelley DE: Subcutaneous abdominal fat and thigh muscle composition predict insulin sensitivity independently of visceral fat. Diabetes 46(10):1579-85, 1997

237. Snyder WS CM, Manssett ES, Larhansen LT, Howells GP, Tipton IH: Report of the Task Group on Reference Man. Oxford, UK:Pergamon. 1975

238. Physical activity and cardiovascular health. NIH Consensus Development Panel on Physical Activity and Cardiovascular Health. Jama 276(3):241-6, 1996

239. Pate RR, Pratt M, Blair SN, Haskell WL, Macera CA, Bouchard C, Buchner D, Ettinger W, Heath GW and King AC: Physical activity and public health. A recommendation from the Centers for Disease Control and Prevention and the American College of Sports Medicine. JAMA 273(5):402-7, 1995

240. Lapidus L, Bengtsson C, Larsson B, Pennert K, Rybo E and Sjostrom L: Distribution of adipose tissue and risk of cardiovascular disease and death: a 12 year follow up of participants in the population study of women in Gothenburg, Sweden. $\mathrm{Br}$ Med J (Clin Res Ed) 289(6454):1257-61, 1984

241. Ross R, Leger L, Marliss EB, Morris DV and Gougeon R: Adipose tissue distribution changes during rapid weight loss in obese adults. Int $J$ Obes 15(11):733-9, 1991

242. Kamel EG, McNeill G and Van Wijk MC: Change in intra-abdominal adipose tissue volume during weight loss in obese men and women: correlation between magnetic resonance imaging and anthropometric measurements. Int J Obes Relat Metab Disord 24(5):607-13, 2000

243. Han TS, Richmond P, Avenell A and Lean ME: Waist circumference reduction and cardiovascular benefits during weight loss in women. Int J Obes Relat Metab Disord 21(2):127-34, 1997 
244. Ross R and Janssen I: Physical activity, total and regional obesity: dose-response considerations. Med Sci Sports Exerc 33(6 Suppl):S521-7; discussion S528-9, 2001

245. Meijer EP, Westerterp KR and Verstappen FT: Effect of exercise training on total daily physical activity in elderly humans. Eur J Appl Physiol Occup Physiol 80(1):16-21, 1999

246. Goran MI and Poehlman ET: Endurance training does not enhance total energy expenditure in healthy elderly persons. Am J Physiol Endocrinol Metab 263(5):E950-957, 1992

247. Goodpaster BH, Katsiaras A and Kelley DE: Enhanced fat oxidation through physical activity is associated with improvements in insulin sensitivity in obesity. Diabetes 52(9):2191-7, 2003

248. Zimmet P, Alberti KG and Shaw J: Global and societal implications of the diabetes epidemic. Nature 414(6865):782-7, 2001

249. Diabetes Atlas. Third edition. Eds. Brussels, International Diabetes Federation, 2007

250. Katzmarzyk PT and Janssen I: The economic costs associated with physical inactivity and obesity in Canada: an update. Can J Appl Physiol 29(1):90-115, 2004

251. Finkelstein EA, Fiebelkorn IC and Wang G: State-Level Estimates of Annual Medical Expenditures Attributable to Obesity. Obesity Res 12(1):18-24, 2004

252. Canadian Guidelines for Body Weight Classification in Adults. 2003

253. Obesity: Preventing and managing the global epidemic. Report of a WHO consultation., 1998

254. Clinical Guidelines on the Identification, Evaluation, and Treatment of Overweight and Obesity in Adults--The Evidence Report. National Institutes of Health. Obes Res 6 Suppl 2:51S-209S, 1998

255. Willis LH, Slentz CA, Houmard JA, Johnson JL, Duscha BD, Aiken LB and Kraus WE: Minimal versus Umbilical Waist Circumference Measures as Indicators of Cardiovascular Disease Risk. Obesity 15(3):753-759, 2007

256. Seidell JC, Cigolini M, Charzewska J, Ellsinger BM and Contaldo F: Regional obesity and serum lipids in European women born in 1948. A multicenter study. Acta Med Scand Suppl 723:189-97, 1988 
257. Katzmarzyk PT, Janssen I, Ross R, Church TS and Blair SN: The Importance of Waist Circumference in the Definition of Metabolic Syndrome: Prospective analyses of mortality in men. Diabetes Care 29(2):404-409, 2006

258. Flegal KM: Waist circumference of healthy men and women in the United States. Int J Obes, 2007

259. Lemieux I, Pascot A, Couillard C, Lamarche B, Tchernof A, Almeras N, Bergeron J, Gaudet D, Tremblay G, Prud'homme D, Nadeau A and Despres JP:

Hypertriglyceridemic waist: A marker of the atherogenic metabolic triad (hyperinsulinemia; hyperapolipoprotein B; small, dense LDL) in men? Circulation 102(2):179-84, 2000

260. Measure your risk, Stay young at heart [article online], 2006. Available from www.obesitynetwork.ca/files/130782_Aventis_Livret_En1385.pdf. Accessed 13 July 2000

261. Carson JAS: Pocket Tape Measure for Waist Circumference: Training Medical Students and Residents on a Simple Assessment of Body Composition. J. Nutr. 133(2):547S-549, 2003

262. Exploring Global Cardiometabolic Risk, Scientific Summary Report [article online], 2006. Available from Accessed 13 July 2000

263. Shen W, Punyanitya M, Chen J, Gallagher D, Albu J, Pi-Sunyer X, Lewis CE, Grunfeld C, Heymsfield SB and Heshka S: Visceral adipose tissue: relationships between single slice areas at different locations and obesity-related health risks. Int J Obes (Lond), 2006

264. Jakicic JM, Winters C, Lang W and Wing RR: Effects of intermittent exercise and use of home exercise equipment on adherence, weight loss, and fitness in overweight women: a randomized trial. JAMA 282(16):1554-60, 1999

265. McGuire MT, Wing RR, Klem ML, Seagle HM and Hill JO: Long-term maintenance of weight loss: do people who lose weight through various weight loss methods use different behaviors to maintain their weight? Int $J$ Obes Relat Metab Disord 22(6):572-7, 1998

266. Luan JA, Wong MY, Day NE and Wareham NJ: Sample size determination for studies of gene-environment interaction. Int J Epidemiol 30(5):1035-40, 2001

267. Hsieh FY, Bloch DA and Larsen MD: A simple method of sample size calculation for linear and logistic regression. Stat Med 17(14):1623-34, 1998 
268. Onat A, Avci GS, Barlan MM, Uyarel H, Uzunlar B and Sansoy V: Measures of abdominal obesity assessed for visceral adiposity and relation to coronary risk. Int $J$ Obes Relat Metab Disord 28(8):1018-25, 2004

269. Goodpaster BH, Krishnaswami S, Resnick H, Kelley DE, Haggerty C, Harris TB, Schwartz AV, Kritchevsky S and Newman AB: Association between regional adipose tissue distribution and both type 2 diabetes and impaired glucose tolerance in elderly men and women. Diabetes Care 26(2):372-9, 2003

270. Howell DC: Statistical Methods for Psychology. 5th. Crockett C, Eds. Pacific Grove, Duxbury, 2002 
Appendix A: Determination of Sample Size and Statistical Power 


\section{Study \#1 - Waist circumference and abdominal adipose tissue distribution: influence of age and sex}

In this study, we wanted to examine differences in the slopes of the association between visceral fat and waist circumference between genders and between older versus younger adults. If we assume standardized $\beta$ coefficients similar to what we have previously reported in men and women $(\beta=0.08$ and 0.04 respectively (228)), we would have a statistical power of 0.60 to detect a significant $(\mathrm{P}<0.05)$ interaction in the slopes $(266)$ in 400 men and women.

\section{Study \#2 - Does Measurement Site for Visceral and Abdominal Subcutaneous Adipose}

\section{Tissue Alter Associations With the Metabolic Syndrome?}

A sample size formula for a two-sample t-test can be used to calculate the required sample size for simple logistic regression models with continuous covariates (267). Based on a previous study in our lab (54), the standard deviation of visceral AT in a similar population of men was $\sim 60 \mathrm{~cm}^{2}$. In studies investigating visceral AT and metabolic risk report a $50 \mathrm{~cm}^{2}$ difference in VAT between those with or without the metabolic syndrome (268) and a $27 \mathrm{~cm}^{2}$ difference between men with normal glucose tolerance and type 2 diabetes (269). Thus, if we assume a difference of $50 \mathrm{~cm}^{2}$ and a variance of $65 \mathrm{~cm}^{2}$ between men with and without the metabolic syndrome, a power calculation assuming a significance value of $5 \%$ would have a statistical power of 0.77 to see group differences if we have 14 subjects with the metabolic syndrome and 71 without (effective sample size of $n=24)(270)$. 


\section{Study \# 3 - Mortality Study:}

This is a preliminary study investigating the relationship between these various adipose tissue depots and mortality we are uncertain what the expected differences between groups would be. Using a similar approach as study \#2, assuming a conservative difference between the decedents and controls of $20 \mathrm{~cm}^{2}$ and a variance of $65 \mathrm{~cm}^{2}$, a power calculation assuming a significance value of $5 \%$ would have a statistical power of 0.86 to see group differences if we have 97 decedent and 184 controls (270).

\section{Study \# 4 - Dose Response to Aerobic Exercise on Adiposity and Metabolic Risk in Postmenopausal Women:}

As this is a subsidiary study, all power calculations were made for the two primary outcome measures $\left(\mathrm{VO}_{2 \max }\right.$ and $\left.\mathrm{SBP}\right)$ of the main research study. All power calculations assumed the treatment effects to be tested at a 5\% significance level. It was determined that adequate power would be achieved with 150,100 , and 100 women exercising in the 4,8 and $12 \mathrm{kcal} / \mathrm{kg} / \mathrm{wk}$ exercise groups, and with 100 women in the control group. Power calculations based on the anticipated increases in $\mathrm{VO}_{2 \max }$ of $7 \%, 12 \%$, and $15 \%$ in the 4,8 and $12 \mathrm{kcal} / \mathrm{kg} / \mathrm{wk}$ exercise groups, respectively, results in statistical power of $0.85,0.99$ and 0.99 , respectively, to see significant increases in $\mathrm{VO}_{2 \max }$ as compared to the control group. Similarly, the anticipated reductions in SBP of 5, 7 and $9 \mathrm{mmHg}$ in the 4,8 and $12 \mathrm{kcal} / \mathrm{kg} / \mathrm{wk}$ exercise groups, respectively, results in statistical power of $0.84,0.98$ and 0.99 , respectively, to see significant increases in SBP as compared to the control group. The test for a significant dose-response trend across the three exercise groups has power of 0.85 .

Post hoc power analyses revealed that in order to achieve significance at $5 \%$ and $80 \%$ power, at least 16 subjects per group (only 11 in the control group) would have been required to achieve statistical significance for visceral fat change relative to control. In 
terms of our major outcome variable, waist circumference analyses in the full sample had a power of $91 \%$. 
Appendix B: Anthropometric Data Collection Form - Queen's University 
Subject Name:

Dates: BIA

Time:

Tester:

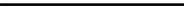

Time:

BIA Weight (kg):

RJL Impedance

$\mathbf{R}$ :

$\begin{array}{ll}\text { R: } & \text { Leg } \\ \text { c: } \quad \text { Leg }\end{array}$

Torso

Arm Whole $\mathrm{R}$

Xc: Whole $\mathrm{R}$

Tanita Leg-Leg

Impedance (Z)

Body Weight

Tanita Segmental

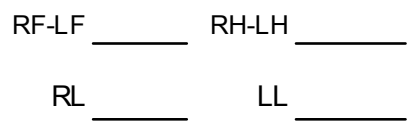

$\mathrm{RH}-\mathrm{RF}$

LH-LF

RA

LA

Anthro Weight (kg):

Stand. Height $(\mathrm{cm})$ :

Arm Length $(\mathrm{cm})$ :

Sitting Height (cm):

Acromion Height (cm):

Skinfolds (m
Subscapular
Tricep
Bicep
Mid-Axillary
lliac Crest
Abdomen
Thigh
Calf

1
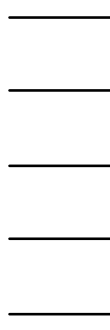

2

3

mean
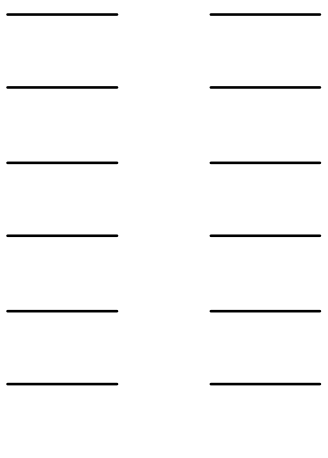
Circumference Measures $(\mathrm{cm})$ :

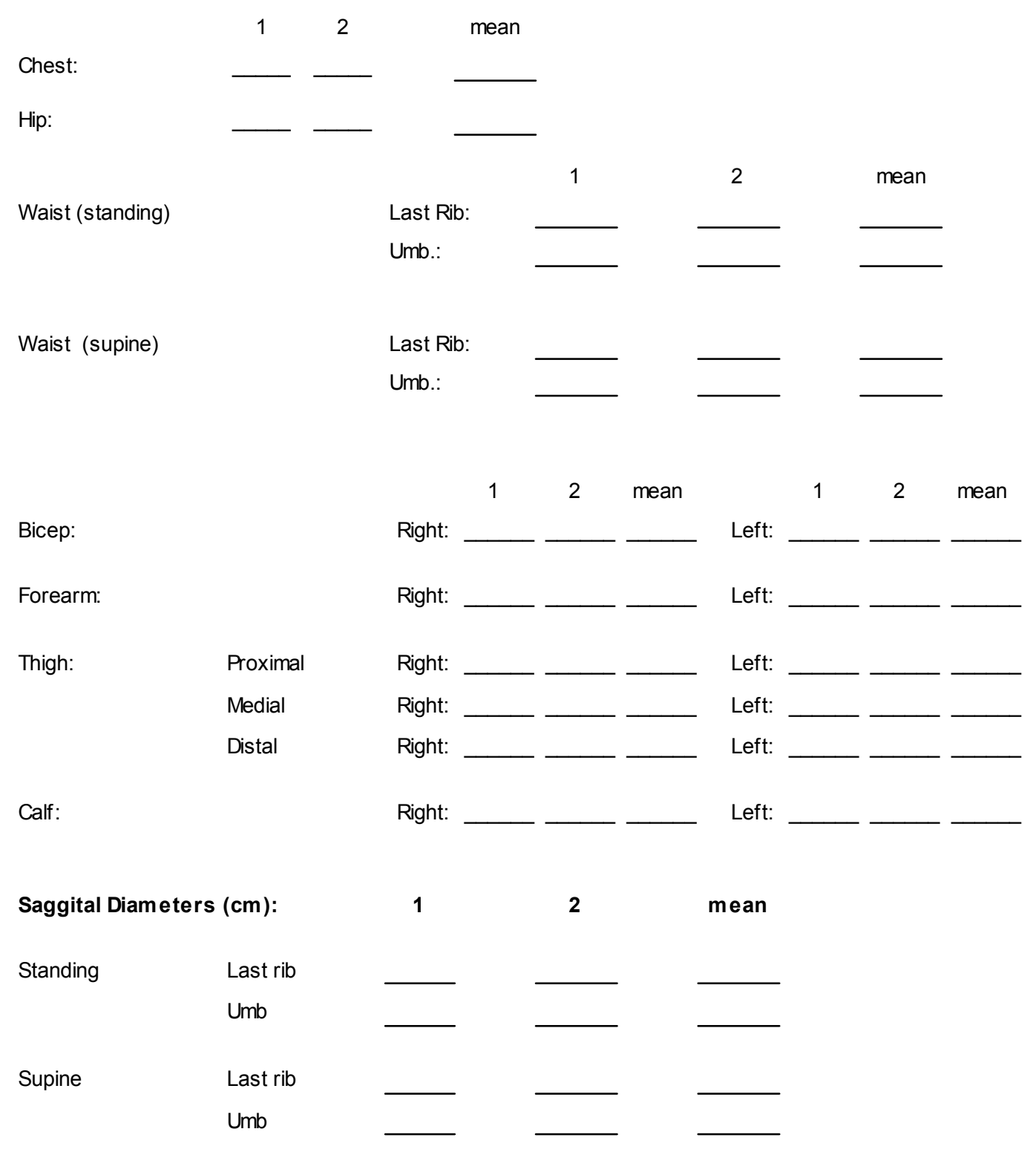


Appendix C: Electron Beam Computerized Tomography Scan Consent Form in Accordance with the Ethical Guidelines of The Cooper Institute Institutional Review Board 
I understand that the information that I am providing below is for the purpose of assessing the effectiveness of measuring and using coronary artery calcium as a predictor of coronary heart disease. I also understand that my records remain confidential, as described in the letter received with this questionnaire, and that the results of this study may be published; however, my name or other identifiers will not be provided. Only group data will be presented.

If you have any questions about your rights as a research study participant, you may contact $\mathrm{Dr}$. Stephen Farrell, Institutional Review Board Chair, at The Cooper Institute (972) 341-3200.

Name: (please print)

Signature: Date:

\section{INSTRUCTIONS}

Please read each question carefully.

Please use a black ink pen to print your information in capital letters and avoid contact with the edge of the box.

\begin{tabular}{|l|l|l|}
\hline $\begin{array}{l}\text { Print letters like this: } \\
\text { Not like this: }\end{array}$ & $\begin{array}{l}\text { Shade circles like this: } \\
\text { Not like this: }\end{array}$
\end{tabular}

Please review your name and address and make any changes below:

Last Name:

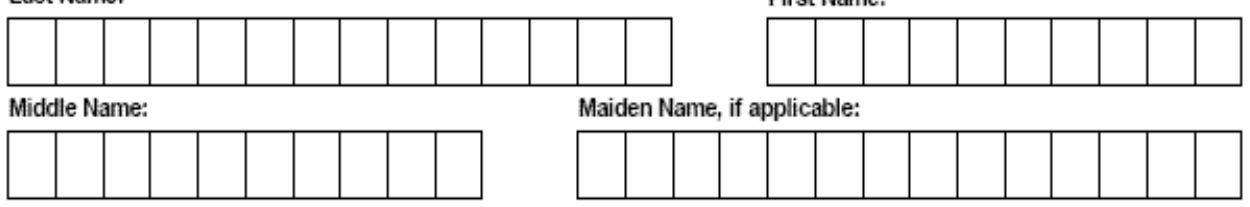

Number \& Street Address:

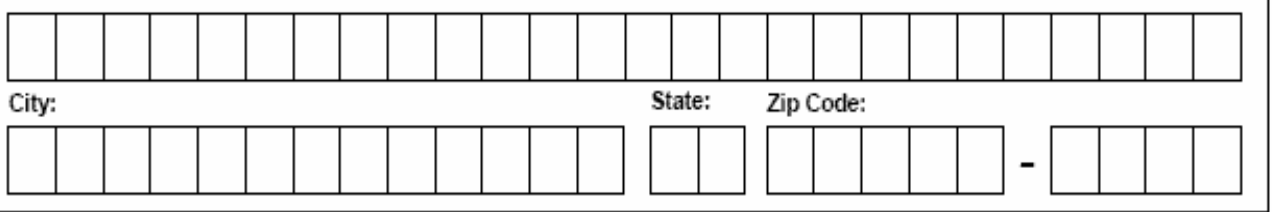

(Area Code) Home Phone Number:

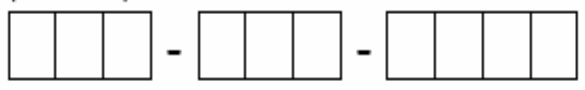

(Area Code) Home Fax Number:

email

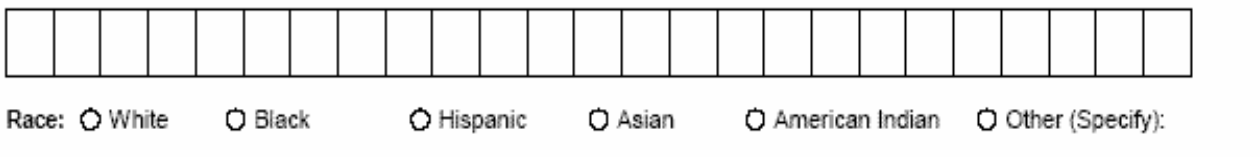




\begin{tabular}{|l|l|l|l|l|l|l|}
\hline What is your current weight in pounds? & What is your current height (feet and inches)? \\
\hline & & & \\
\hline
\end{tabular}

Have you ever had or been toid by a physician that you had any of the illnesses or conditions listed below? If so,
please indicate the year of diagnosis.
Congestive heart failure
Angina (heart pain)
High blood pressure (hypertension)
Heart attack

Have you ever had the following procedures? If so, please indicate the year of the procedure.

Carotid artery surgery

Coronary bypass surgery

Coronary angioplasty (balloon, stent) $\frac{\text { Yes } \mathrm{Ne}}{\mathrm{O} O}$

00

00
Year
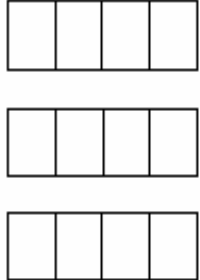

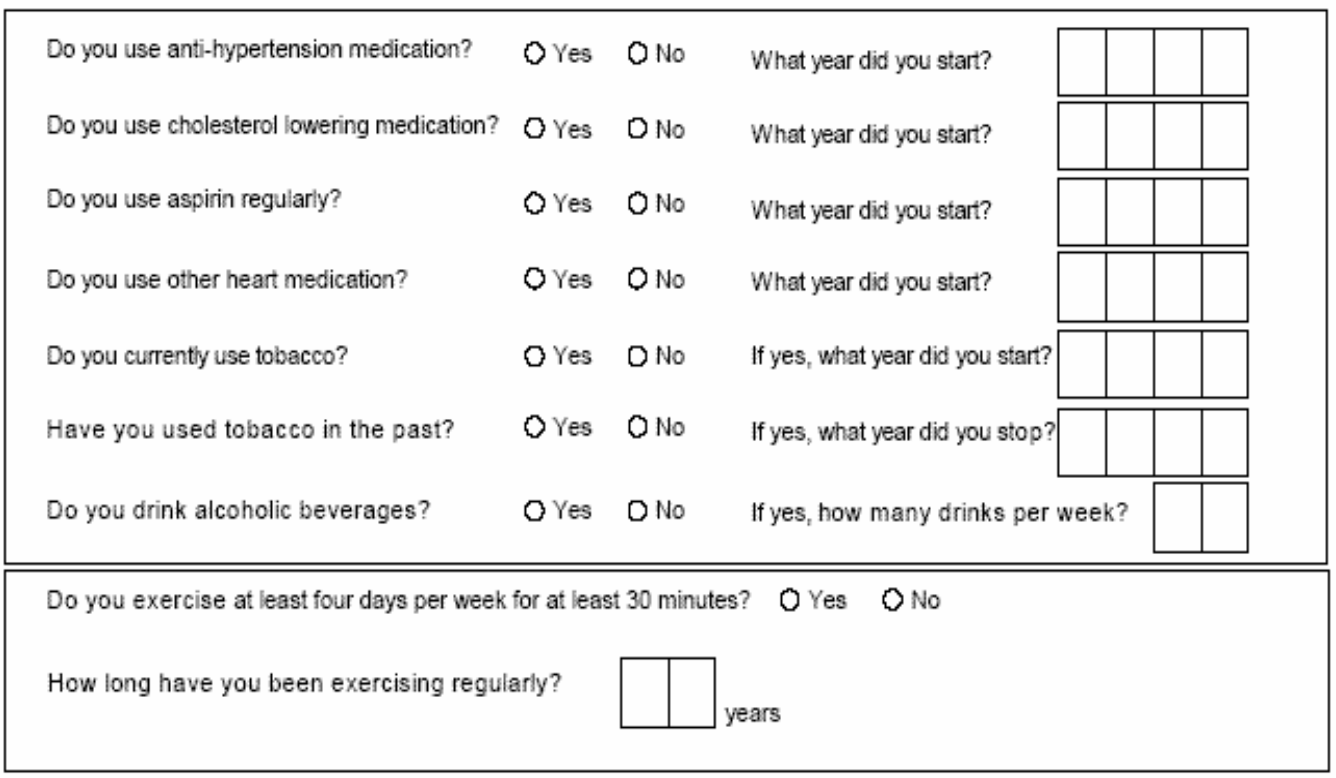


Appendix D: Aerobics Center Longitudinal Study Self-Report Questionnaire 


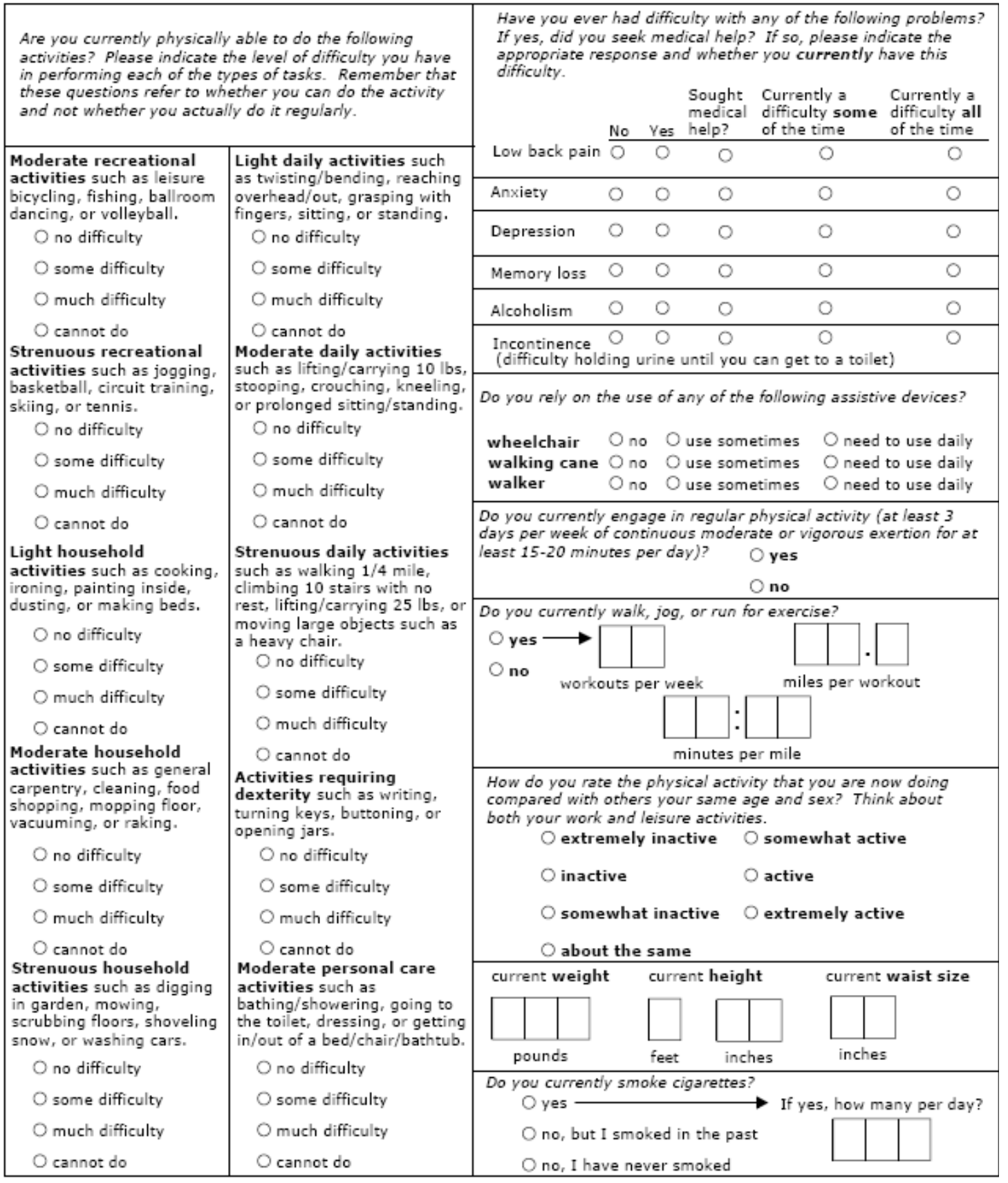




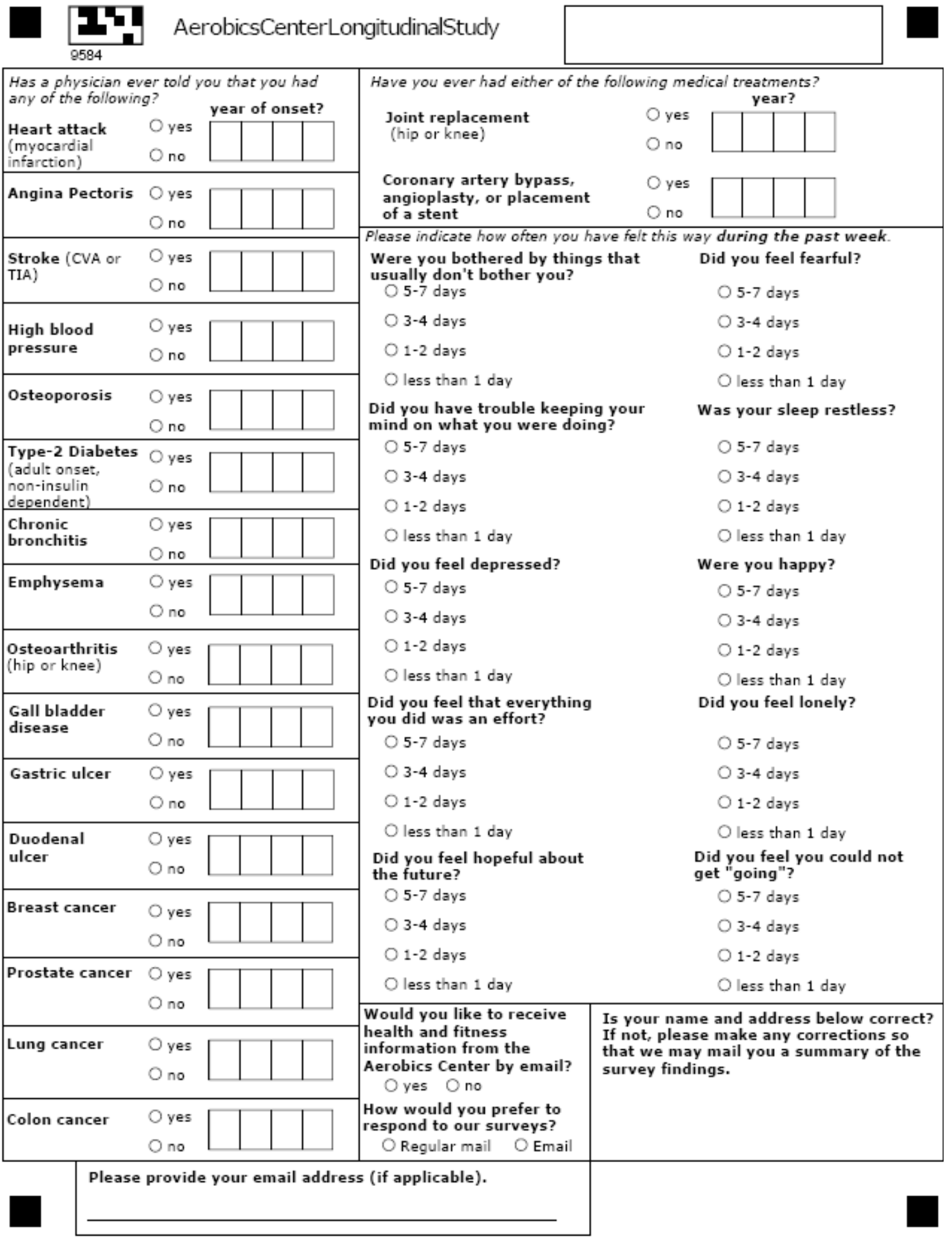


Appendix E: DREW Consent Form - The Cooper Institute 
Informed Consent Form

\author{
Participant Consent Form for Medical Testing \\ DREW: Dose Response to Exercise in Women Aged 45-75
}

\author{
The Cooper Institute \\ 12330 Preston Road \\ Dallas TX 75230
}

\begin{abstract}
You were selected as a candidate for this study because you are currently physically inactive, between the ages of 45 and 75 years old, post-menopausal, have no known major health problems, and are available for regular exercise sessions at The Cooper Institute. The following information describes the study and your role as a participant. The investigators will answer any questions you may have about the study, this consent form, and your participation in the project. Please read this form carefully and do not hesitate to ask questions about any aspect of the study.
\end{abstract}

\title{
Purpose
}

This study will test the effectiveness of different amounts of exercise on improving fitness and selected cardiovascular disease risk factors. Well known risk factors include elevated blood pressure, decreased fitness, elevated blood cholesterol, elevated blood glucose, and obesity.

\section{Procedures}

Individuals who agree to participate and who meet all eligibility criteria will be randomly assigned to one of four groups. Random assignment means your group assignment is determined by chance, and you will not be able to choose one group over the others.

Individuals will be assigned to 1 of 4 groups, Group A, B, C, or D.

1) Group A - Two-week lifestyle intervention focused on diet, weight loss, smoking cessation, physical activity, and stress management.

2) Group B - Two-week lifestyle intervention focused on diet, weight loss, smoking cessation, physical activity, and stress management. Exercise 3 to 4 times per week for approximately 20 minutes per session for 6 months.

3)Group C - Two-week lifestyle intervention focused on diet, weight loss, smoking cessation, physical activity, and stress management. Exercise 3 to 4 times per week for approximately 40 minutes per session for 6 months.

4)Group $D$ - Two-week lifestyle intervention focused on diet, weight loss, smoking cessation, physical activity, and stress management. Exercise 3 to 4 times per week for approximately 60 minutes per session for 6 months.

(initials) I understand I will not be able to pick my group and that assignment will be random. 
The 2-week lifestyle intervention focusing on the above mentioned topics will occur after orientation and before the medical and laboratory assessments begin. It includes a total of 6 sessions at The Cooper Institute lasting approximately 45 minutes each.

All study participants will receive a series of medical, health, and functional tests at the beginning of the study (baseline) and after 6 months (follow-up). You will be asked to complete questionnaires concerning your physical activity, food intake, health history, medication usage, and quality of life issues at the baseline and 6-month follow-up testing. In addition, you will be asked to complete a brief health screening at 2 months and 4 months.

The baseline and follow-up testing will require 3 sessions each. In the first session, you will undergo a brief physical examination by a licensed physician or physician assistant, have your blood pressure measured, do deep breathing relaxation while we monitor your heart rate through a modified electrocardiogram (ECG) and have a blood draw. Some of the blood will be stored for future tests or assays. If you do not wish for your blood to be stored as part of this study, please initial the box "Do not take additional blood for storage and future testing" below. In preparation for these blood tests, you will be asked to fast (to stop eating and drinking anything except water for 12 hours) before coming in for this visit.

Do not take additional blood for storage and future testing.

Each of the remaining two test sessions will last approximately 60 minutes and will consist of body fat composition measurement and 2 maximal exercise stationary bike test. When you arrive for your visits, we will record your resting heart rate and measure your blood pressure. During the exercise bike test, you will have your heart rate monitored by a machine called an electrocardiogram (ECG) and you will wear a nose clip and a mouthpiece to monitor your breathing and oxygen use. Your percentage of body fat will be estimated by measuring skinfold thickness and by using a tape measure to record waist and hip measurements.

In addition to the measurements described above, you may be invited to participate in one or more ancillary studies that are part of the overall project. Participation in an ancillary study will involve having measurements such as additional body composition and fat distribution, intramuscular fat, bone mineral density, or additional blood chemistry analyses. These additional tests will be at no cost to you, and you will be informed of the results. Before participating in any ancillary study, you will have the opportunity to have the specific benefits and risks of the study explained to you, and you will have an opportunity to ask questions about the ancillary study. You will be asked to sign an additional informed consent for the ancillary study. Participation in any ancillary study will be completely voluntary, and refusing to volunteer for an ancillary study will not affect participation in the primary study.

\section{Discomforts and Risks}

There are some potential (possible) discomforts and risks associated with participating in DREW. You may experience temporary pain during the blood drawing, with later bruising at the puncture site. Only specially trained staff will be responsible for 
collection of blood samples. (Also, any samples collected during the course of the study will be tested for biological hazards if an incident occurs that may result in exposure of any individual to potentially hazardous material.) There exists the possibility of certain changes occurring during the bicycle test. These include abnormal blood pressure, fainting, abnormal heart beats, and, in rare instances, heart attack, stroke, and death. Every effort is made to minimize these risks by reviewing information about your health and fitness before the test and by closely observing you during the testing procedure. Emergency equipment and trained personnel are available to deal with unusual situations that may arise. Some abnormalities may require you to obtain additional medical follow-up and/or exclude your participation in the study.

There may be some discomfort in the beginning of the study from increasing your physical activity. The possibilities include, but are not limited to, some muscle and joint stiffness. This stiffness generally subsides in 1 or 2 days, and is not considered to be serious. You might experience an exercise-related injury such as a strain, sprain, or other injury to your muscles or joints. There also is a risk of heart attack and sudden death during exercise. In studies of the U.S. population, it has been reported that for every 396,000 hours of jogging, there is one death. In healthy populations that have been medically screened, the risk of sudden death from jogging is even less. There may be other risks that are currently not foreseeable.

\section{Exclusions}

If you have a history of heart disease, stroke, angina, uncontrolled or insulin-dependent diabetes, lung, or kidney disease, if you have been a participant in a previous clinical trial on physical activity at our center, or plan to move from the area in the next year, you will be excluded from the study. Also, if you have any other condition that would make participation in physical activity unwise or unsafe, you will be excluded.

\section{Alternatives to Participation}

To determine your blood levels of cholesterol, glucose, and other blood factors, or to receive other health screening evaluations, you could visit your personal health care provider. You may choose to increase your activity level on your own without enrolling in this study. You may choose to work out at a fitness center or employ your own exercise trainer without enrolling in this study.

\section{Voluntary Participation}

Participation in the DREW study is voluntary. Refusal to participate will involve no loss of benefits to which you are entitled. Further, you may withdraw from the study at any time without penalty. If you decide to withdraw from the study, we request that you notify the Project Director, Dr. Tim Church, of your decision in writing. At that time, we also request that you complete an exit interview by mail or telephone to determine the reason for withdrawing from the study.

\section{Significant Findings}

You will be told of any significant findings that may occur during the course of this study that could relate to your willingness to continue to participate. A report of your clinical results will be given to you approximately 60 days following your 6 -month measurement 
visit. If you wish, a report also can be sent to your physician for your medical file if we have your signed request and your physician's name and address on file.

\section{Removal from Study}

At the discretion of the Project Director, subjects may be taken out of this study due to unanticipated circumstances. The investigator and the sponsor reserve the right to terminate the study and discontinue your participation at any time for any reason in order to ensure your safety.

Some possible reasons for withdrawing a subject from the study:

- failure to follow instructions

- the investigator decides that continuation could be harmful to you

- you need treatment not allowed in the study

- the study is canceled

- other administrative reason

\section{Benefits to Participants}

There is no cost for participation in this study. You will receive health and medical screening examinations and the results will be discussed with you. You will have the opportunity to participate in an exercise program with professional guidance. Studies have shown that people who exercise at levels prescribed by this program will reduce their risk of heart disease and some forms of cancer, and increase their feelings of psychological well being.

\section{Compensation}

All participants who complete the scheduled measurements at baseline and at 6 months will be eligible for a $\$ 500$ payment. However, participants in the exercise groups (groups B, C, \& D) must have a $90 \%$ or better adherence rate to receive the full $\$ 500$. The payment will be reduced by $\$ 50$ for each week of missed sessions. The minimum amount you will receive will be $\$ 150$. There will be opportunities for make-up of individual sessions missed, although there will be a limit on the number of make-up sessions because of the limitations on the amount of exercise you may accumulate in a week. You will be allowed to miss a total of 10 exercise sessions. You will be allowed a total of 16 unsupervised sessions for extenuating circumstances. If you can make up the missed sessions within 2 weeks, the supervised sessions will be extended. Legally, you can be paid only if you are a U.S. citizen, a legal resident alien (i.e., possess a "green" card), or have a work-eligible visa sponsored by the paying institution.

\section{Project Funding}

The U.S. Public Health Service (USPHS), National Heart, Lung, and Blood Institute (NHLBI), and the National Institutes of Health $(\mathrm{NIH})$ fund this project.

\section{Compensation for Medical Treatment}

If you suffer any adverse experience during the testing, study staff will render first aid and emergency care. The project physician will not provide medical care outside test and exercise supervision. You will need to obtain follow-up care through your primary physician for any concerns that arise as a result of or during the course of the study. 
Further, The Cooper Institute is not responsible for compensation of care for concerns that arise as a result of or during the course of the study. Upon completion of the study, any further medical assessment and testing will need to be performed by your primary physician.

\section{Confidentiality}

Only you and the investigators will have access to your study records and other data obtained from the study, as required by the Privacy Act, 5, U.S.C. 522a. Details from your medical records will be stored on a private computer with an identification number, but your name will not be used as an identifier. Information stored on the computer may be seen by DREW clinic study staff or government staff at the USPHS, NHLBI, and NIH, which fund the study. Your name will not appear in any publications; only group data will be used. If for any reason you desire to share any and/or all your data with someone else, a signed letter stating your desire to release this information and to whom it should be released will be required.

\section{Questions}

Your signature indicates that the investigators or other members of study staff have answered all your questions about the study and your participation in the study. If you should have additional questions during the course of the study, or if any problems arise, you should contact the Principal Investigator, Dr. Steven Blair, at 972-341-3200 or Project Director, Dr. Tim Church, at 972-341-3200. If you have questions about your rights as a research study participant, contact Dr. Steve Farrell, the Institutional Review Board Chairman at The Cooper Institute (972-341-3200).

\section{Bill of Rights}

As a study participant, you have the following rights. These rights include but are not limited to your right to:

be informed of the nature and purpose of the experiment;

be given an explanation of the procedures to be followed in the medical experiment, and any drug or device to be utilized;

be given a description of any attendant discomforts and risks reasonably to be expected;

be given an explanation of any benefits to you reasonably to be expected, if applicable;

be given a disclosure of any appropriate alternatives, drugs, or devices that might be advantageous to you, their relative risks and benefits;

be informed of the avenues of medical treatment, if any available to you after the experiment, if complications should arise;

be given an opportunity to ask questions concerning the experiment or the procedures involved; 
be instructed that consent to participate in the medical experiment may be withdrawn at any time and that you may discontinue participation without prejudice;

be given a copy of the signed and dated consent form;

and be given the opportunity to decide to consent or not to consent to a medical experiment without the intervention of any element of force, fraud, deceit, duress, coercion, or undue influence on your decision.

\section{Authorization}

You are making a decision whether or not to participate in this study. You should not sign until you understand all the information presented in the previous pages and all your questions about the research have been answered to your satisfaction. Your signature indicates that you have decided to participate after having read (or been read) the information provided above.

I understand that I am not waiving any legal rights or releasing the local institution sponsoring this study or its agents from liability for negligence. I understand that in the event of physical injury resulting from the research procedures, the local institution sponsoring this study does not have funds budgeted for compensation either for lost wages or for medical treatment. Therefore, aside from the emergency care previously described, the local institution does not provide for treatment or reimbursements for such injuries.

Name (Please print.)

Signature

Date

Witness

Date 
Appendix F: DREW Electron Beam Tomography Disclosure and Consent Form The Cooper Institute 


\section{The Cooper Institute \\ Research Using Electron Beam Tomography Disclosure and Consent Form}

TO THE PARTICIPANT: You have the right to be informed about the risks and hazards in the medical procedure to be used. This disclosure is simply an effort to make you better informed so you may give or withhold your consent to the procedure.

\section{CONSENT}

I voluntarily consent and authorize the Cooper Clinic to administer an electron beam tomography (EBT) scan for research purposes.

I realize that there is radiation exposure associated with this procedure. The exposure is about equivalent to living in Denver (altitude of approximately 5600 feet) for one year. I understand the scan is for research purposes only and will consist of a limited number of pictures or cross-sections from the thigh and abdomen. I understand there will be no scanning of my heart, thorax or pelvis. Thus the scan will be of no diagnostic or screening value. Further I understand that this scan will not be read by a radiologist. In the unlikely event that a gross abnormality is found during measurement of research variables, I understand that further testing and/or diagnostic procedures may be needed to further evaluate the findings. I do understand that such tests and/or procedures may entail additional costs for which I am responsible.

I have been given opportunity to ask questions about this procedure and the risk and hazards involved and I believe that I have sufficient information to give and informed consent. I certify this form is clear to me that I have read it or had it read to me and I understand its contents.

Study Participant's Signature

Date: Time:

Witness Signature

Date: Time: 
Appendix G: Letter to DREW Participants Regarding Randomization - The Cooper Institute 


\section{Dear DREW participant,}

As you know, today you will find out what group you have been randomly assigned to. In the DREW study, there are four groups and you could be placed into any one of them as already described in your informed consent. Your group assignment is completely random. This means that your medical assessment results do not determine your group placement, and neither you nor the study staff can choose your group assignment. Once you have been randomized to a group, you cannot be moved from one group to another. Regardless of your group assignment, we ask you to you come in again in 2 months and 4 months to measure your heart rate while you are relaxing. Each visit takes only 30 minutes.

Over the next 6 months, we also will ask you to wear a step counter, keep an activity log, and complete questionnaires dealing with medical symptoms. The activity $\log$ is very similar to the one that you received during the orientation session. The packet you are receiving today contains the activity logs and medical symptoms questionnaires for the entire 6 months and everything is pre-dated for your convenience. If you are assigned to group A, please bring your completed paperwork to your 2- and 4 month visits. If you are assigned to group B, C, or D, please retum your paperwork to the exercise staff as you complete them.

The four groups are as follows:

Group A - You have already received the Health Education sessions so essentially you are on your own for the next 6 months. If you would like to start an exercise program. please feel free to do so. If you do start an exercise program, we ask that you keep a detailed record of what you are doing. For example, if you begin a walking program, it would be very helpful if you could provide us with the distance you wall and how long it takes you to walk that distance. At the end of the study, after your follow up medical assessments, you will be eligible for up to 2 months of supervised exercise at our facility.

Persons in groups B, C, and D will come to the Institute 3 to 4 times per week. The time allotted for the exercise sessions are a rough estimate. Each group has a specific number of calories that need to be expended during each exercise session. Depending on your fitness level your sessions may be longer than we have estimated. The lower your individual fitness, the longer your sessions will take to complete.

Group B - Each session is roughly 20 minutes

Group C - Each session is roughly 40 minutes

Group D - Each session is roughly 60 minutes

If you are assigned to an exercise group, please do not engage in exercise outside of the study. The DREW study is looking at the effect of specific doses (or amounts) of exercise on your fitness, blood pressure, and cholesterol. If you engage in more exercise than what you are assigned during randomization, it does negatively affect the study results.

Do you have any questions or concerns about participating?

Do you have any difficulty walking on a treadmill or riding a bike? Yes No Comments: 
Appendix H: DREW Data Acquisition Timeline Form - The Cooper Institute 


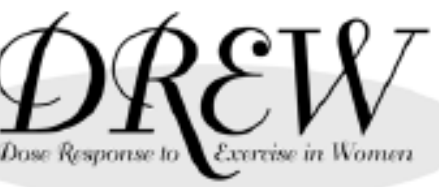

Acrostic:

ID:

\begin{tabular}{|c|c|c|c|c|c|}
\hline \multicolumn{3}{|l|}{ Baseline 1} & \multicolumn{3}{|l|}{ Time: } \\
\hline & Date & Initials & & Date & Initials \\
\hline $\begin{array}{l}\text { Ancillary } \\
\text { Informed } \\
\text { Consents }\end{array}$ & & & Blood work & & \\
\hline HRV & & & Physical exam & & \\
\hline Blood pressure & & & Body composition & & \\
\hline \multicolumn{3}{|l|}{ Baseline 2} & \multicolumn{3}{|l|}{ Time: } \\
\hline & Date & Initials & & Date & Initials \\
\hline Resting ECG & & & \multicolumn{3}{|c|}{$\square$ Measure Blood pressure } \\
\hline \multicolumn{6}{|l|}{ Bike test 1} \\
\hline \multicolumn{3}{|l|}{ Baseline 3} & \multicolumn{3}{|l|}{ Time: } \\
\hline & Date & Initials & & Date & Initials \\
\hline Bike test 2 & & & Digiwalker & & \\
\hline Randomization & & & \multicolumn{3}{|l|}{ D 12 lead EKG } \\
\hline \multicolumn{3}{|l|}{ Follow up 1} & \multicolumn{3}{|l|}{ Time: } \\
\hline & Date & Initials & & Date & Initials \\
\hline Paperwork & & & Blood work & & \\
\hline HRV & & & Physical exam & & \\
\hline Blood Pressure & & & Body composition & & \\
\hline Follow Up 2 & & & Time: & & \\
\hline Blood draw & & & Resting ECG & & \\
\hline Blood pressure & & & Bike test 3 & & \\
\hline \multicolumn{3}{|l|}{ Follow up 3} & \multicolumn{3}{|l|}{ Time: } \\
\hline & Date & Initials & & Date & Initials \\
\hline Bike test 4 & & & \multicolumn{3}{|l|}{$\square 12$ lead EKG } \\
\hline Exit interview & & & & & \\
\hline
\end{tabular}

\begin{tabular}{|l|l|l|l|l|l|l|}
\hline FSH & & & & $\begin{array}{l}\text { Medical } \\
\text { Documentation }\end{array}$ & & \\
\hline Estradiol & & & & & & \\
\hline Carroll & YES & NO & & & & \\
\hline
\end{tabular}


Appendix I: DREW Anthropometric Data Form - The Cooper Institute 

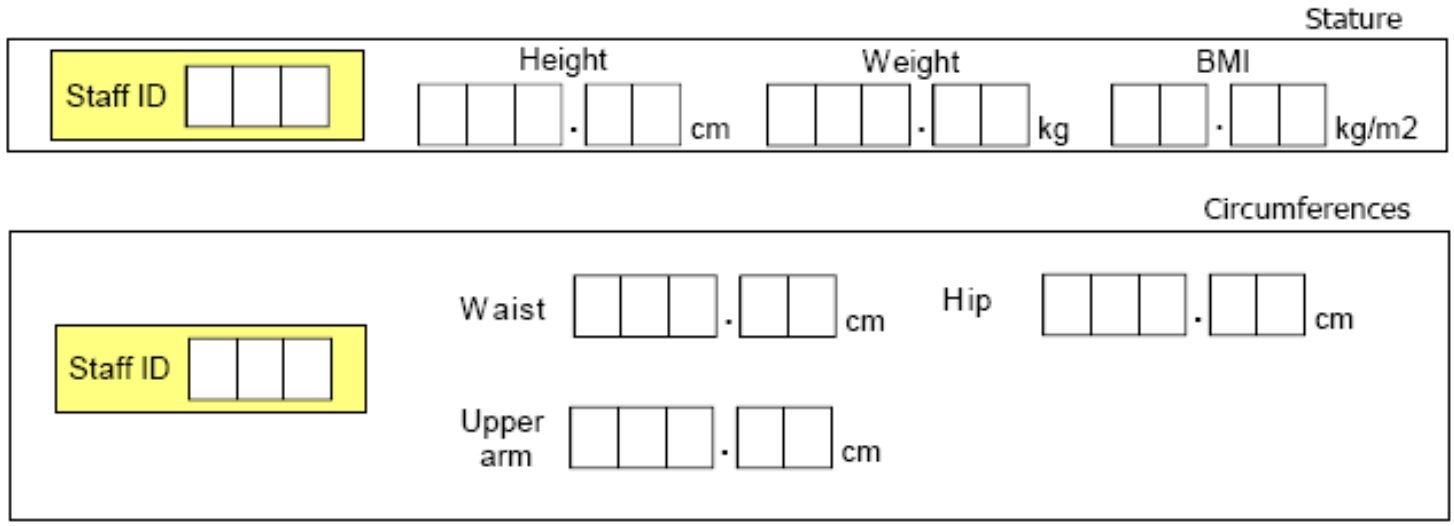

Length

Staff ID

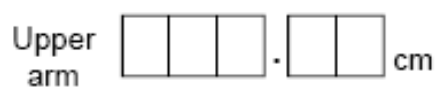

Skinfolds

\begin{tabular}{|c|c|c|c|}
\hline Biceps & $\mathrm{mm}$ & $\mathrm{mm}$ & $\mathrm{mm}$ \\
\hline Triceps & $\mathrm{mm}$ & $\mathrm{mm}$ & $\mathrm{mm}$ \\
\hline Midaxillary & $\mathrm{mm}$ & $\mathrm{mm}$ & $\mathrm{mm}$ \\
\hline Subscapular & $\mathrm{mm}$ & $\mathrm{mm}$ & $\mathrm{mm}$ \\
\hline Abdominal & $\mathrm{mm}$ & $\mathrm{mm}$ & $\mathrm{mm}$ \\
\hline Suprailiac & $\mathrm{mm}$ & $\mathrm{mm}$ & $\mathrm{mm}$ \\
\hline Thigh & $\mathrm{mm}$ & $\mathrm{mm}$ & $\mathrm{mm}$ \\
\hline Calf & $\mathrm{mm}$ & $\mathrm{mm}$ & $\mathrm{mm}$ \\
\hline
\end{tabular}

$\boldsymbol{\square}$\begin{tabular}{|l|l|l|l|l|l|l|}
\hline$\square$ & & id & $\square$ & id & acrostic & $\square \square$ visit code \\
\hline
\end{tabular}


Appendix J: DREW Maximal Oxygen Uptake Test Form - The Cooper Institute 


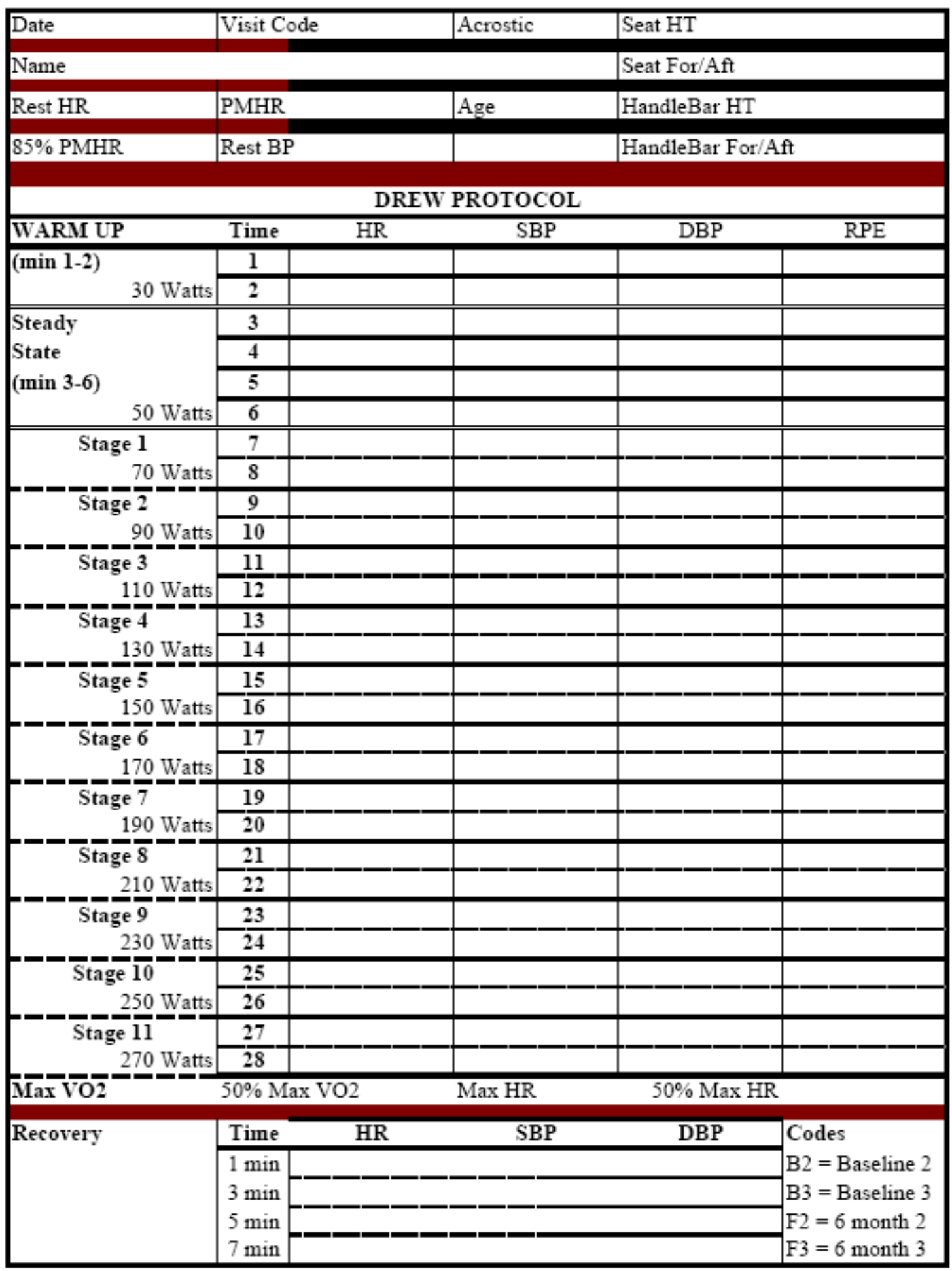


Appendix K: DREW Intervention Exercise Log - The Cooper Institute 


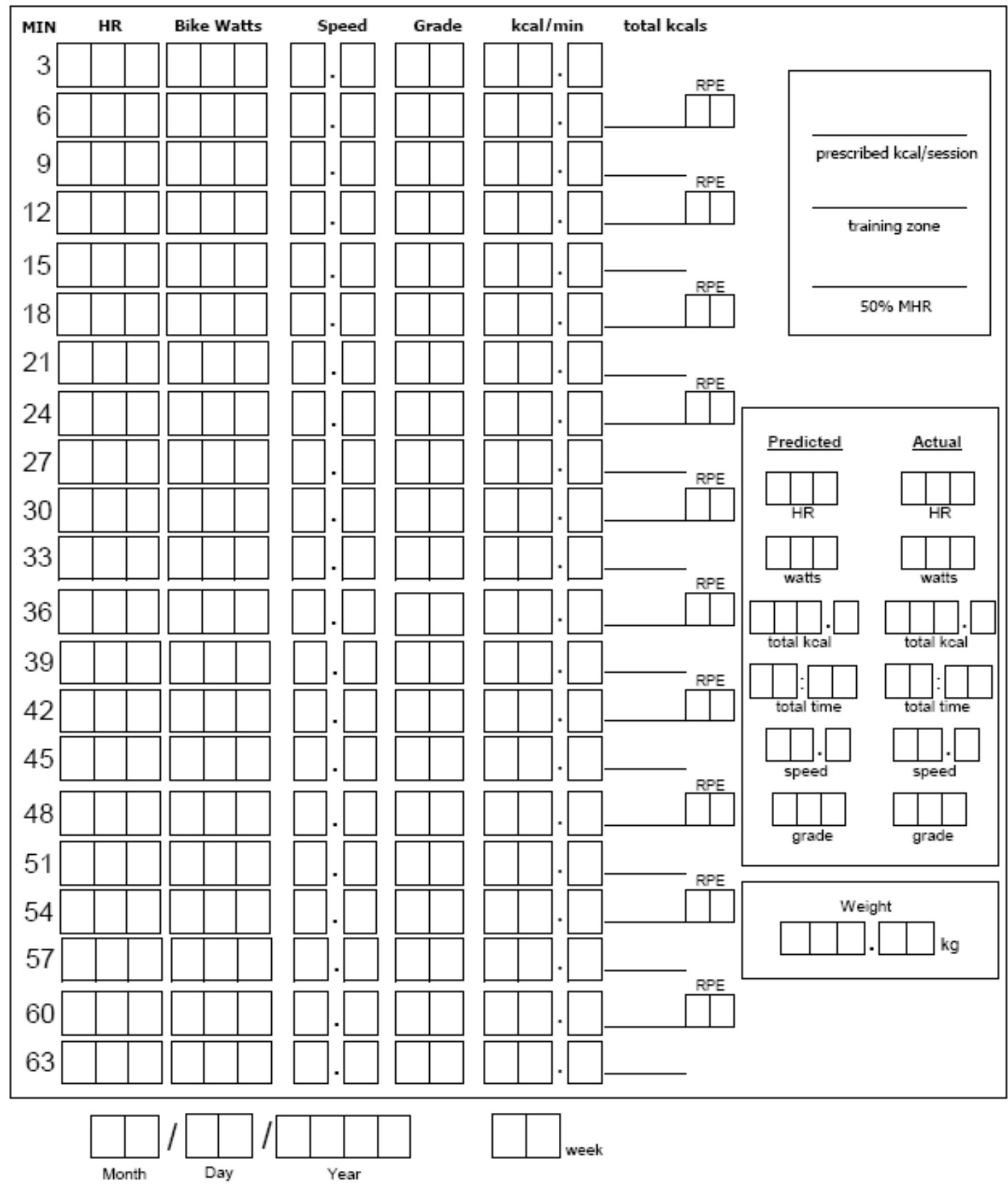

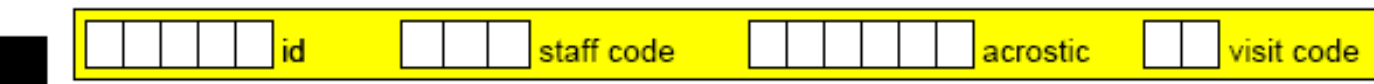


- בקיק:
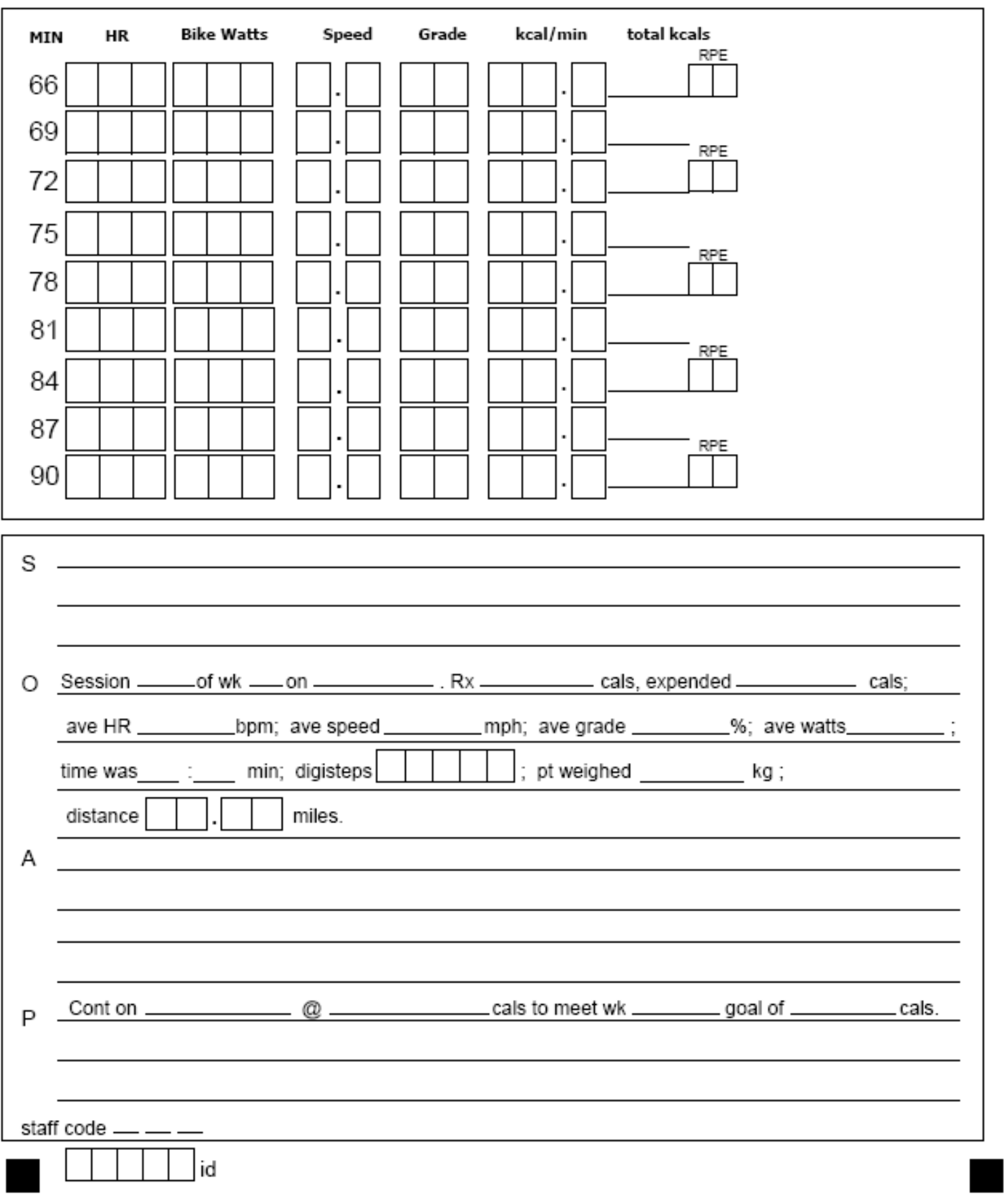


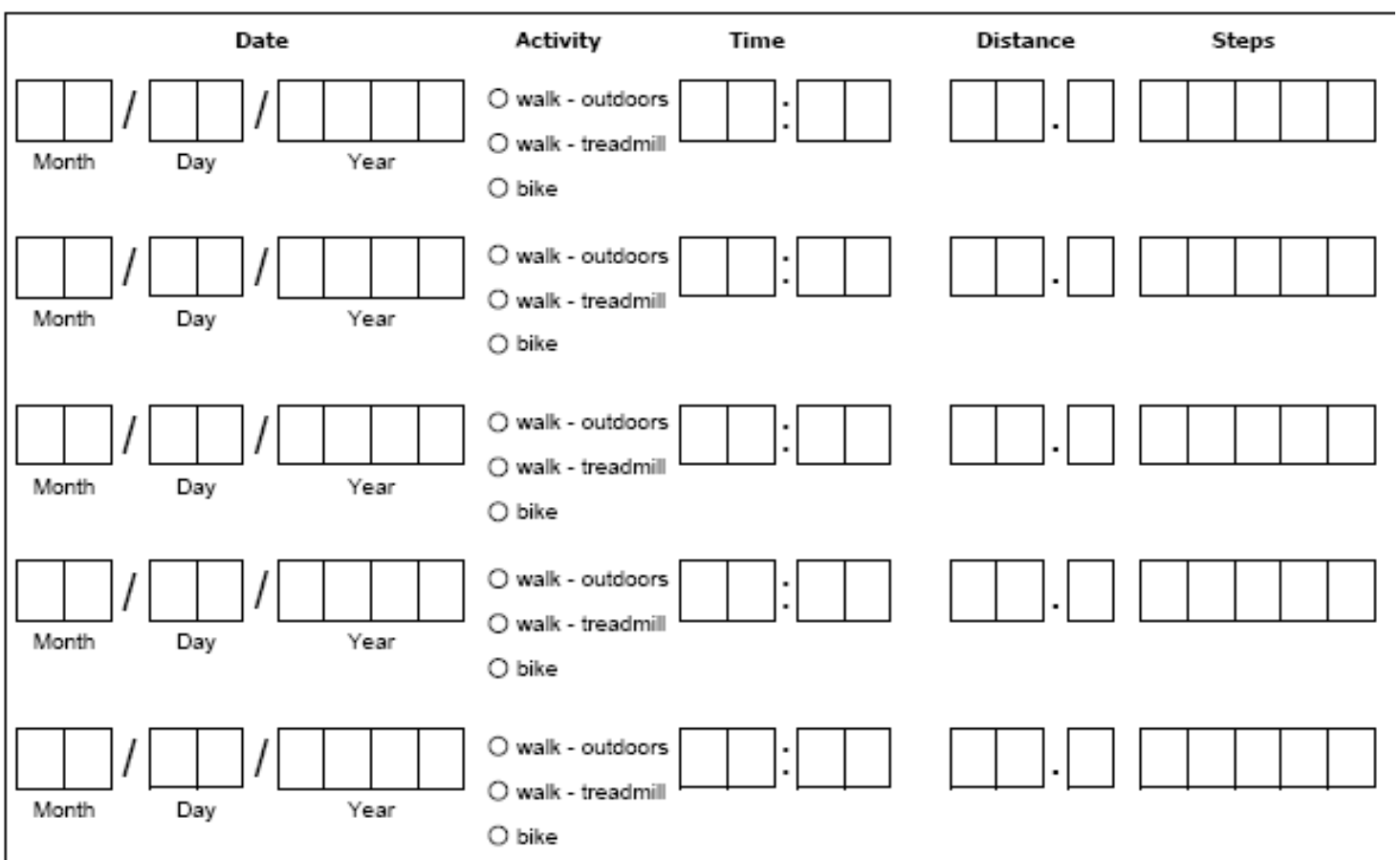

For your exercise session, you can choose any of the following activities:

\begin{abstract}
Walking (outside)
Walking (outside)

Walking (treadmill)
\end{abstract}

Biking

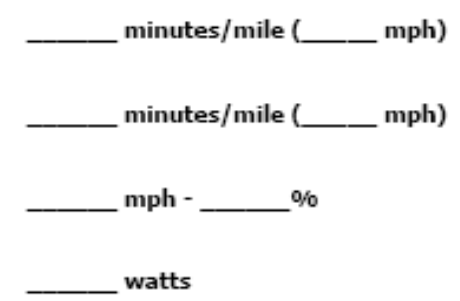

mph)

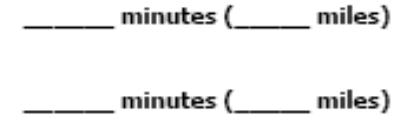

minutes

minutes

Please complete sessions during your absence.

If you have any questions, please contact me by phone [(972)-341-3244] or email [ajordan@cooperinst.org]. Please keep a record of your exercise and bring this sheet with you when you return to the lab.

Thanks, Alex Jordan

\begin{tabular}{|l|l|l|l|l|l|l|l|l|l|l|l|l|}
\hline & & \\
\hline
\end{tabular}


Appendix L: Examples of Statistical Analyses Used in Manuscript 1 to 4 


\section{Example of a Multiple Linear Regression with Interaction Variables in SAS Used in Manuscript 1}

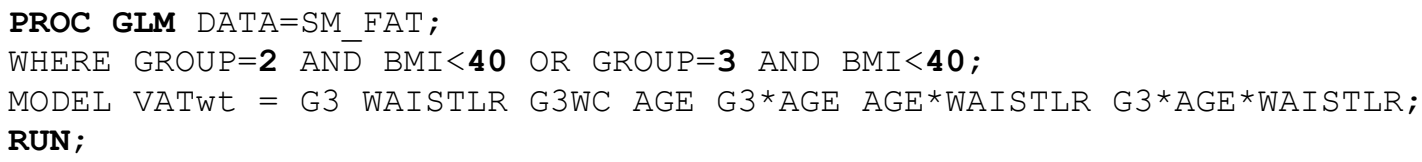

Dependent Variable: VATwt

The GLM Procedure

$\begin{array}{lr}\text { Source } & \\ \text { Model } & \text { DF } \\ \text { Error } & \\ \text { Corrected Total } & \\ & \\ & \text { R-Square } \\ & 0.728838 \\ \text { Source } & \\ \text { G3 } & \\ \text { WAISTLR } & \\ \text { G3WC } & \\ \text { AGE } & \\ \text { G3*AGE } & \\ \text { WAISTLR*AGE } & \\ \text { G3*WAISTLR*AGE } & \\ \text { Source } & \\ \text { G3 } & \\ \text { WAISTLR } & \\ \text { G3WC } & \\ \text { AGE } & \\ \text { G3*AGE } & \\ \text { WAISTLR*AGE } & \\ \text { G3*WAISTLR*AGE } & \end{array}$

\begin{tabular}{|c|c|c|c|c|}
\hline DF & $\begin{array}{r}\text { Sum of } \\
\text { Squares }\end{array}$ & Mean Square & F Value & $\mathrm{Pr}>\mathrm{F}$ \\
\hline 7 & 199.3209522 & 28.4744217 & 92.92 & $<.0001$ \\
\hline 242 & 74.1566401 & 0.3064324 & & \\
\hline 249 & 273.4775923 & & & \\
\hline
\end{tabular}

Coeff Var Root MSE VATwt Mean

DF Type I SS

$1 \quad 2.7236015$

185.7930115

1.6886420

2. 6729035

2. 1694708

2. 3072744

1.9660484

\section{Type III SS}

1.14216673

0.03196831

1.53610634

2.65242695

1.45271644

4.01745953

1.96604844

$\begin{array}{rl}\text { F Value } & \text { Pr }>\text { F } \\ 8.89 & 0.0032 \\ 606.31 & <.0001 \\ 5.51 & 0.0197 \\ 8.72 & 0.0035 \\ 7.08 & 0.0083 \\ 7.53 & 0.0065 \\ 6.42 & 0.0119 \\ & \\ \text { F Value } & \text { Pr }>\text { F } \\ 3.73 & 0.0547 \\ 0.10 & 0.7470 \\ 5.01 & 0.0261 \\ 8.66 & 0.0036 \\ 4.74 & 0.0304 \\ 13.11 & 0.0004 \\ 6.42 & 0.0119\end{array}$

Mean Square

1.14216673

0.03196831

1.53610634

2. 65242695

1.45271644

4.01745953

1.96604844

6.42

0.0119

Standard

Error

Estimate

0.139017401

$-8.149291419$

0.004470262

0.110220164

WAISTLR

G3WC

$-0.098750146$

G3*AGE

0.148412356

WAISTLR*AGE

G3*WAISTLR*AGE
0.001363717

$-0.001996348$
1.19722373

4.22106885

0.01384014

0.04922863

0.03356475

0.06816273

0.00037663

0.00078815
t Value
0.12
$-1.93$
0.32
2.24
$-2.94$
2.18
3.62
$-2.53$

\section{Final Equations}

$\operatorname{VAT}_{\text {Pre Women }}(\mathrm{kg})=-0.14+0.0045(\mathrm{WC})-0.099($ Age $)+0.0014($ Age*WC$)$, $\operatorname{VAT}_{\text {Post Women }}(\mathrm{kg})=-8.01+0.115(\mathrm{WC})+0.050($ Age $)-0.0006\left(\right.$ Age $\left.^{*} \mathrm{WC}\right)$. 


\section{Example of a Multiple Logistic Regression in SAS Used in Manuscript 2+3}

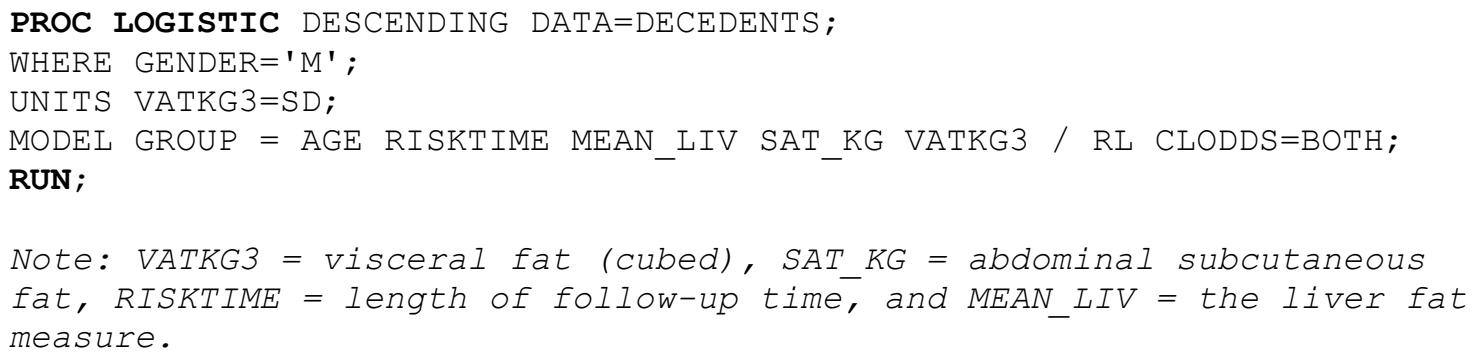

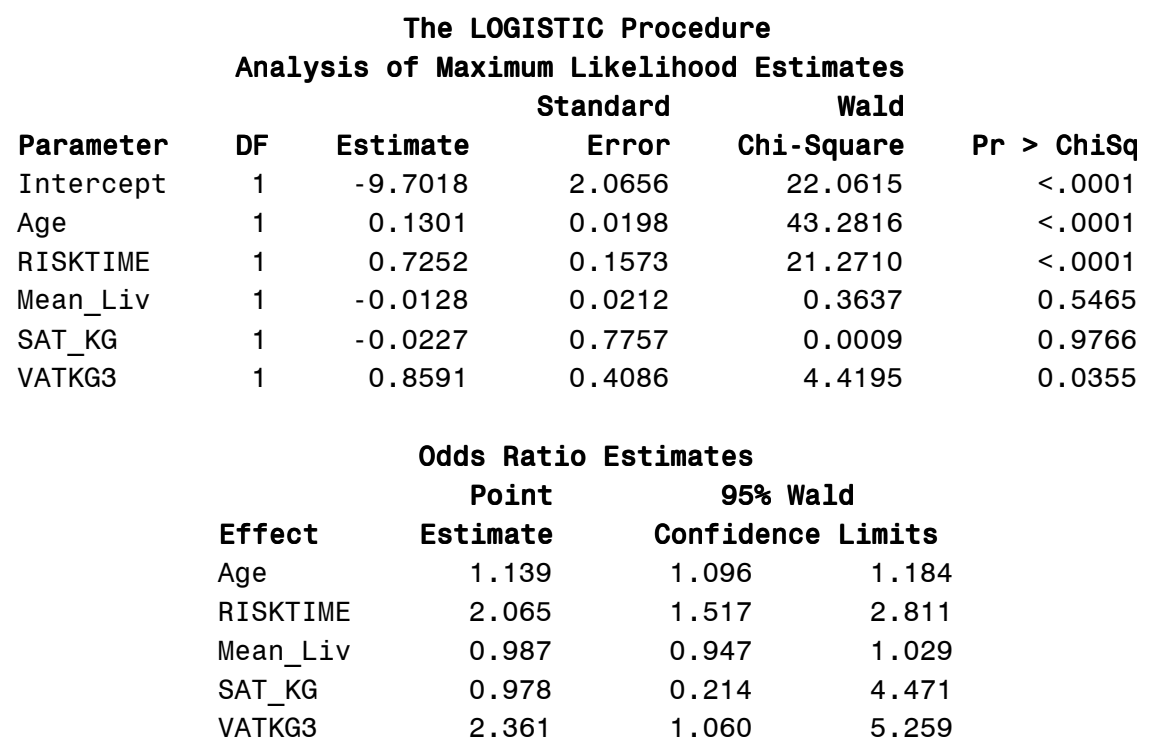

Association of Predicted Probabilities and Observed Responses

$\begin{array}{lrll}\text { Percent Concordant } & 88.1 & \text { Somers' D } & 0.764 \\ \text { Percent Discordant } & 11.7 & \text { Gamma } & 0.765 \\ \text { Percent Tied } & 0.1 & \text { Tau-a } & 0.340 \\ \text { Pairs } & 17112 & \text { C } & 0.882\end{array}$

\begin{tabular}{lcccc} 
Profile Likelihood Confidence Interval for Adjusted Odds Ratios \\
Effect & Unit & Estimate & $95 \%$ Confidence Limits \\
VATKG3 & 0.6888 & 1.807 & 1.080 & 3.267 \\
\multicolumn{5}{c}{ Wald Confidence Interval for Adjusted Odds Ratios } \\
Effect & Unit & Estimate & $95 \%$ Confidence Limits \\
VATKG3 & 0.6888 & 1.807 & 1.041 & 3.137
\end{tabular}




\section{Example of an ANCOVA in SAS Used in Manuscript 4}

Testing for interaction effects before conducting ANCOVA analysis.

Note: WAISTCM6 is the waist measurement at follow-up, WAISTCM0 is the waist measurement at baseline, and GRP is the group assignment (ie. control, $4 \mathrm{kcal} / \mathrm{kg} / \mathrm{week}$, etc.)

PROC GLM; CLASS GRP;

MODEL WAISTCM6 = GRP WAISTCMO GRP*WAISTCMO;

RUN;

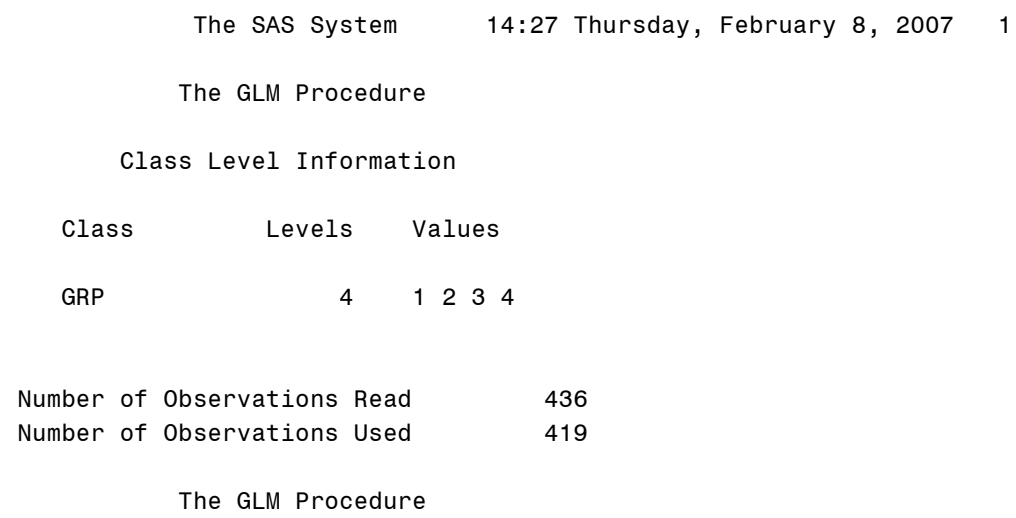

Dependent Variable: WaistCm6 WaistCm6

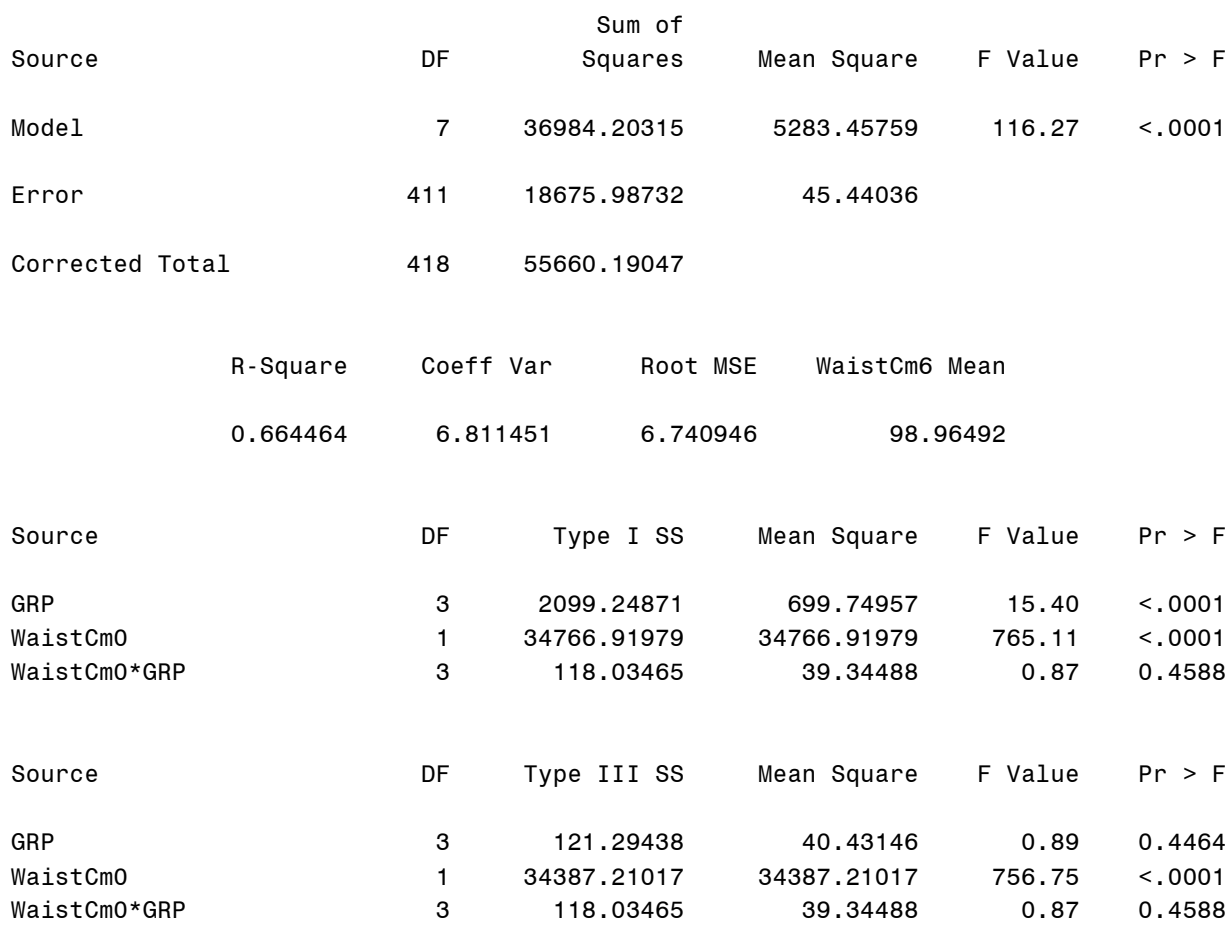


No Significant Interaction, therefore proceed with ANCOVA adjusted least-squared means analyses.

\section{PROC GLM;}

CLASS GRP;

MODEL WC_C = GRP WAISTCMO;

MEANS GR $\bar{P}$; LSMEANS GRP / STDERR TDIFF;

RUN ;

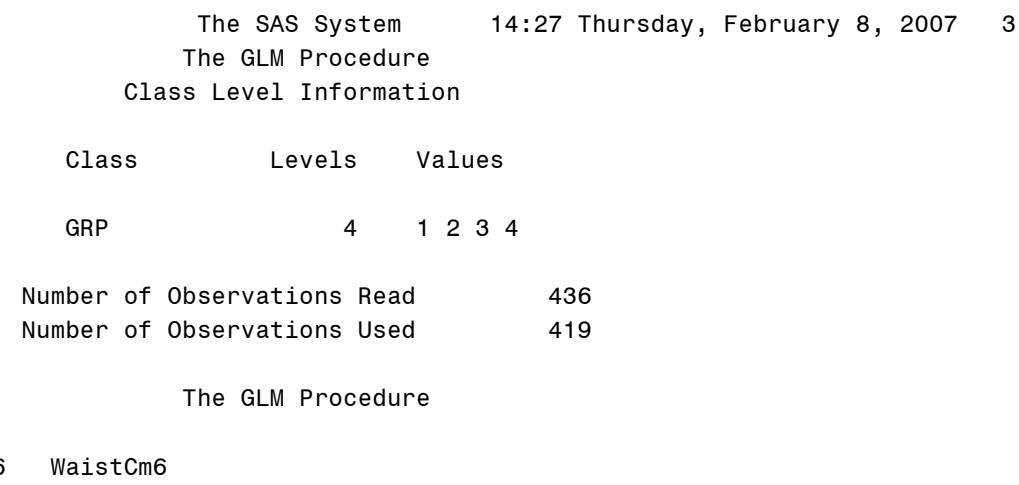

\begin{tabular}{|c|c|c|c|c|c|}
\hline \multicolumn{6}{|c|}{ Sum of } \\
\hline Source & DF & Squares & Mean Square & F Value & $\mathrm{Pr}>\mathrm{F}$ \\
\hline Model & 4 & 36866.16850 & 9216.54212 & 203.02 & $<.0001$ \\
\hline Error & 414 & 18794.02198 & 45.39619 & & \\
\hline Corrected Total & 418 & 55660.19047 & & & \\
\hline
\end{tabular}

$\begin{array}{lrrr}\text { R-Square } & \text { Coeff Var } & \text { Root MSE } & \text { WaistCm6 Mean } \\ 0.662344 & 6.808139 & 6.737669 & 98.96492\end{array}$

\begin{tabular}{|c|c|c|c|c|c|}
\hline Source & DF & Type I SS & Mean Square & F Value & $\mathrm{Pr}>\mathrm{F}$ \\
\hline GRP & 3 & 2099.24871 & 699.74957 & 15.41 & $<.0001$ \\
\hline WaistCm0 & 1 & 34766.91979 & 34766.91979 & 765.86 & $<.0001$ \\
\hline Source & DF & Type III SS & Mean Square & F Value & $\mathrm{Pr}>\mathrm{F}$ \\
\hline GRP & 3 & 701.77124 & 233.92375 & 5.15 & 0.0017 \\
\hline WaistCm0 & 1 & 34766.91979 & 34766.91979 & 765.86 & $<.0001$ \\
\hline
\end{tabular}

The GLM Procedure

\begin{tabular}{|c|c|c|c|c|c|}
\hline \multicolumn{2}{|c|}{ Level of } & \multicolumn{2}{|c|}{ - _ - - WaistCm6 - _ } & \multicolumn{2}{|c|}{ 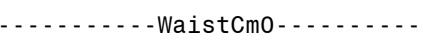 } \\
\hline GRP & $\mathrm{N}$ & Mean & Std Dev & Mean & Std Dev \\
\hline 1 & 94 & 102.750106 & 12.2143764 & 102.744149 & 12.0395090 \\
\hline 2 & 142 & 97.488028 & 10.3071172 & 100.269014 & 11.2405117 \\
\hline 3 & 87 & 99.568851 & 12.2987481 & 102.403448 & 11.8109079 \\
\hline 4 & 96 & 96.895833 & 11.0969500 & 99.075000 & 11.1863639 \\
\hline
\end{tabular}


The GLM Procedure

Least Squares Means

$\begin{array}{rrrrr}\text { GRP } & \begin{array}{r}\text { WaistCm6 } \\ \text { LSMEAN }\end{array} & \begin{array}{r}\text { Standard } \\ \text { Error }\end{array} & \mathrm{Pr}>|\mathrm{t}| & \begin{array}{r}\text { LSMEAN } \\ \text { Number }\end{array} \\ 1 & & & & \\ 2 & 101.360832 & 0.696748 & <.0001 & 1 \\ 3 & 98.063428 & 0.565795 & <.0001 & 2 \\ 4 & 98.450012 & 0.723484 & <.0001 & 3 \\ & 98.418999 & 0.689860 & <.0001 & 4\end{array}$

Least Squares Means for Effect GRP

t for HO: LSMean $(i)=\operatorname{LSMean}(j) / \operatorname{Pr}>|t|$

Dependent Variable: WaistCm6

$i / j$

1

1

2

2.903817

0.0039

$-0.42049$

0.6743

0.42049

0.6743

0.399105

0.6900

$-0.03095$

0.9753

NOTE: To ensure overall protection level, only probabilities associated with pre-planned comparisons should be used.

NOTE: Group $1=$ Control

Group $2=4 \mathrm{kcal} / \mathrm{kg} /$ week

Group $3=8 \mathrm{kcal} / \mathrm{kg} /$ week

Group $4=12 \mathrm{kcal} / \mathrm{kg} /$ week

Treatment effects only different from control. No differences between treatment effects on waist circumference change. 
Appendix M: Unrelated Publications 


\title{
Exercise-Induced Reduction in Obesity and Insulin Resistance in Women: a Randomized Controlled Trial
}

\author{
Robert Rass, *f Ian Janssen, *f Jody Dawson, *Ann-Marie Kungl * Jennifer L. Kuk, Suzy L. Wong, * \\ Thanh-Binh Nguyen-Duy, * Salung Lee, * Katherine Kilpatrick, and Robert Hudson'
}

\begin{abstract}
ROSS, ROBERT, IAN JANSSEN, JODY DAWSON, ANN-MARIE KUNGL, JENNIFER L. KUK, SUZY L. WONG, THANH-BINH NGUYEN-DUY, SOTUNG LEE, KATHERINE KILPATRICK, AND ROBERT HUDSON.

Exercise-induced reduction in obesity and insulin resistance in women: a randomized controlled trial. Obes Res. 2004; 12:789-798.

Objectives: To determine the effects of equivalent diet- or exercise-induced weight loss and exercise without weight loss on subcutaneous fat, visceral fat, and insulin sensitivity in obese women.

Research Methods and Procedures: Fifty-four premenopausal women with abdominal obesity [waist circumference $110.1 \pm 5.8 \mathrm{~cm}$ (mean \pm SD)] (BMI $31.3 \pm 2.0 \mathrm{~kg} / \mathrm{m}^{2}$ ) were randomly assigned to one of four groups: diet weight loss $(n=15)$, exercise weight loss $(n=17)$, exercise without weight loss $(n=12)$, and a weight-stable control group $(n=10)$. All groups underwent a 14-week intervention.

Results: Body weight decreased by $\sim 6.5 \%$ within both weight loss groups and was unchanged in the exercise without weight loss and control groups. In comparison with controls, cardiorespiratory fitness improved within the exercise groups only $(p<0.01)$. Reduction in total, abdominal, and abdominal subcutaneous fat within the exercise weight lo5s group was greater $(p<0.001)$ than within all
\end{abstract}

lloxived Bor review Seplanter 11, 2006

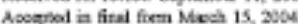

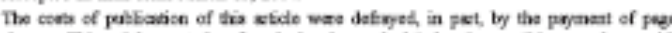

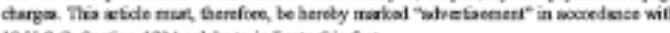

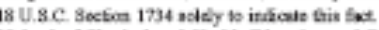

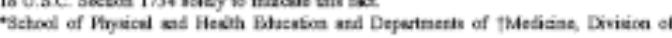

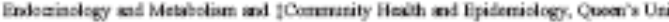

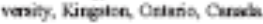

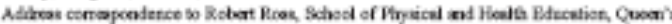

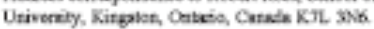

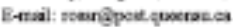

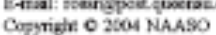

other groups. The reduction in total and abdominal fat within the diet weight loss and exercise without weight loss groups was greater than within controls $(p<0.001)$ but not different from each other $(p>0.05)$. Visceral fat decreased within all treatment groups $(p<0.008)$, and these changes were not different from each other. In comparison with the control group, insulin sensitivity improved within the exercise weight loss group alone $(p<0.001)$

Discussion: Daily exercise without caloric restriction was associated with substantial reductions in total fat, abdominal fat, visceral fat, and insulin resistance in women. Exercise without weight loss was also associated with a substantial reduction in total and abdominal obesity.

Key words: weight loss, exercise, visceral fat, abdominal fat, insulin resistance

\section{Introduction}

The escalation in the prevalence of obesity in both men and women $(1,2)$ represents a major public health problem and reinforces the need to develop effective treatment strategies. Although caloric restriction remains the comerstone of obesity reduction strategies $(3,4)$, we bave recently reported that exercise-induced weight loss is associated with a greater reduction in total body fat, a preservation of lean tissue mass, and an increase in cardiorespiratory fimess in comparison with equivalent diet-induced weight loss in men and that exercise without weight loss is associated with significant reductions in abdominal obesity (5). Little is known about the utility of exercise alone (e.g., no calonic restriction) as a strategy for obesity reduction in women. Although some exercise studies have included women, few prescribed an exercise program for which one would expect meaningful weight loss (6). A rationale that would support the exclusion of women in studies where exercise is performed for longer duration (e.g, $45 \mathrm{~min} / \mathrm{d}$ ) is not known. To the contrary, when performing moderate-intensity exercise, 


\title{
Liver Fat Is Not a Marker of Metabolic Risk in Lean Premenopausal Women
}

\author{
Jennifer L. Kuk, Milton Z. Nichaman, Timothy S. Church, Steven N. Blair, and Robert Ross
}

We examined the independent associations among abdominal adipose tissue (AT) depots, liver fat, cardiorespiratory fitnes: (CRF), and metabolic risk factors in 86 lean premenopausal women. We measured abdominal AT and liver fat by computec tomography (CT), and CRF by a maximal treadmill exercise test. Liver fat was not related to any abdominal AT depot metabolic risk factor, or CRF $(P>.10)$. Visceral AT mass (kilograms) remained a significant $(P<.05)$ predictor of total cholesterol (TC), low-density lipoprotein cholesterol (LDL-Cl, TC/high-density lipoprotein cholesterol (HDL-C), and LDL-C. HDL-C after statistical adjustment for CRF. Abdominal subcutaneous AT mass was also a significant $(P<.05)$ correlate of $\mathrm{TC} / \mathrm{HDL}-\mathrm{C}$ and LDL-C/HDL-C after control for CRF. Visceral AT remained a significant predictor $(P<.05)$ of TC and LDL-C aftel control for abdominal subcutaneous AT. Conversely, subcutaneous AT did not remain a significant correlate after control fol visceral AT. However, the deep subcutaneous AT depot remained significantly associated with LDL-C, TC/HDL-C, anc LDL-C/HDL-C after control for visceral AT. In contrast, visceral AT remained correlated with triglycerides (TG) alone, after control for the deep subcutaneous AT. These observations suggest that liver fat is not a determinant of metabolic risk in lear women. Conversely, both visceral and the deep subcutaneous depot are determinants of metabolic risk in premenopausa woman despite the absence of obesity.

e 2004 Elsevier lic. All rights reserved.

Q EVERAL RECENT studies suggest that fat accumulation $\mathbf{S}$ in the liver is a component of fat discribution that explains variation in metabolic risk independent of abdominal and visceral adiposity. . $^{-3}$ Indeed, Seppala-Lindroos et al' reported that despite similar amouts of visceral adipose tissue (AT), men with bigh liver fat have a greater metabolic risk than those with low liver fat. These findings are consistent with Ngryen-Dury et al, ${ }^{3}$ who recently observed that liver fat was a significant conelate of fasting glucose and plasuna triglycerides (TG) independent of visceral AT. Similarly, Tiikkainen et al2 reported that liver fat is a significant predictor of fasting insulin and TG levels independert of visceral and abdominal subcutaweous AT in obese wowen with previous gestational diabetes. Together, these studies suggest that liver fat is indeed a predictor of metabolic risk independent of abdouninal adiposity. However, the studies reporting liver fat as an independeut predictor of metabolic risk bave been conducted in poptlations with a wide range of abdoninal adiposity, or bounogeneous groups of obese individuals.

Based on these observations, we reasoned that if liver fat was in fact related to metabolic risk independent of obesity, then variation in liver fat might well predict metabolic risk in a sample of lean women characterized by low levels of adiposity, in particular, visceral fat. If this were true, it would reinforce the notion that liver fat is an independent component of abdominal fat distribution and an antecedent for the developanent

From the School of Pipysical and Hoaith Education; and Doparnent of Madicine, Division of Endocrinology and Motabolism, Qunen's Uninersiny, Kingston, Outrovo, Canada; and the Centers for Intograted Health Research, The Cooper Institute, Dallas, $T X$

Submitsad October 16, 2003; accapted Fobruary 20, 2004.

Supporsed in part by research grants from the National hustimtes of Hoaith to SN.B. (AG06945) and MZN. (HL62505), and from the Canadion Lustinutes of Hoalth Resaarch to RR (MT13448).

Adhess reprint reguests to Robert Ross, PhD, School of Physical and Heaith Education, Quevn's University, Kingston, Ontario, Cmada, $K Z \mathrm{~L} 3 \mathrm{~N} 6$.

- 2004 Elsaier Inc All righrs resenved

0026-0495,04/5305-0004\$30 000

dot:10.1016j.metabol. 2004.02 .016 metabolic risk. Furtbermore, no study has considered whether liver fat remained a significant predictor of metabolic risk after segmentation of abdominal subcutaneous AT into its superficial and deep AT conpartments. This is relevant as it has been reported that the more metabolically active deep subcutaneous AT depot predicts metabolic risk independent of visceral AT:

To consider this hypothesis we investigated the independen relationships among all abdominal AT depots, liver fat, cardio respiratory fitwess, and features of the metabolic syndrome in : group of lean, premenopausal women characterized by lon levels of total and abdominal obesity.

\section{MATERIALS AND METHODS}

\section{Subjects}

Subjects consisted of a subset of 86 lean premeaopausal womer selected from a larger cohort who received a medical evamination a the Cooper Clinic in Dollas, TX, between 1995 and 2002. Inclusion criterin recuired that the subjects were nousmokers, bad received a conputed tomography (CT) scan of the abdomival region, self-reportes regular menstual cycles, and were not using oral contraceptives. Fur. ther, levm individuals in this stady were defaed as having a body mos: inder (BMI) less than $25 \mathrm{kgm}^{2}$ and a waist circumference of less than $88 \mathrm{~cm}$. Subjects were from a middle to upper socioecocomic back. ground. Exclusion criterin included persons with history of diabete: mellisis, cardiovascular disease, strobe, cancer, abmonnal resting of evercise electrocardiograns (BCG), or failure to rench at lesst $85 \%$ o their age-precicted maximal heart rate duing the trendmill test. Sab. jects taking medication to trent hypertension and those with higl cholesterol were also excluded. All sabjects gave their filly informe: writen consent prior to participation in the evamination accorcing to the ethical gucideimes of The Cooper Instiste Instituriousl Reviev Bourd, and the smody was reviewed and approwed awanly.

\section{Clinical Examination}

In the moning following an overnight fint of at lenst 12 hours, study participants conpleted a consrehensive medical examination Th: evaluation ivcluded a physical examination, a questionsire on demo graphic factors and henlth habits (alcohol consimiption, cigaretre smok. ing, physical activity, family history, awd metication), antleopometi and blood pressure mensuremeots, blood chenustry analyses, resting ECG, a standardized maximal treadmill test, and a CT scan of the abdominsl region. Body weight and height were measured using a standard physicin's scale and stadiometer, and were used to calculatt 


\section{Cardiorespiratory Fitness Aftenuates Metabolic Risk Independent of Abdominal Subcutaneous and Visceral Fat in Men}

SoJUNG LeE, MSC ${ }^{1}$

JeNnIFER L. Kuk, usc ${ }^{1}$

Peter T. Katzmarzyk, PhD ${ }^{1,2}$
STEVEN N. BLAIR, PED ${ }^{3}$

Timothi S. ChURCH, MD, PHD

ROBERT Ross, PHD $^{1.4}$

OBJECTIVE - Moderate to high levels of cardiorespiratory fitness (CRF) are associated with a bwer risk of the metabolic syndrome and all-cause mortality. Unknown is whether CRF attenuates health risk for a given level of abdominal visceral fat, subcutaneous fat, and/or waist circumference.

RESEARCH DESIGN AND METHODS - The sample studied comprised 297 apparently healthy men with avallable computed tomography or magnetic resonanoe imaging scans of the abdomen, metabolic data, and maximal treadmill exercise test results. Men were categorized into low-CRF (20\%, $n=56)$, moderate-CRF ( $40 \%, n=94)$, and high-CRF $(40 \%, n=147)$ groups based on age and exercise test results. All analyses were adjusted for age.

RESULTS - For a given level of waist circumference, visceral fat, or subcutaneous fat, the high-CRF group had lower triglyceride levels $(P<0.05)$ and higher HDL cholesterol levels than the bw- or moderate-CRF groups. There was a significant group interaction $(P<0.01)$ for blood pressure, indicating that the increase in blood pressure per unit increase in visceral fat or waist circumference was greater in men in the low-CRF group compared with the high-CRF group. The relative nisks of having the metabolic syndrome were $1.8(95 \% \mathrm{CI} 1.0-3.1)$ and $1.6(0.9-$ $2.7)$ times higher in the low- and moderate-CRF groups, respectively, compared with the highCRF group after adjusting for age, visceral fat, and subcutaneous fat ( $P$ for trend $=0.06$ )

CONCLUSIONS - High levels of CRF are associated with a substantial reduction in health risk for a given level of visceral and subcutaneous fat.

Diabetes Care 28:895-901, 2005

$\mathrm{t}$ is well established that abdominal adiposity is a strong predictor of morbid-

ity and mortality independent of BMI $(1,2)$. It is also reported that high levels of cardiorespiratory fitness (CRF) are associated with lower risk of metabolic syndrome (3) and all-cause mortality (4-6).

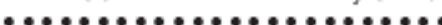

From the ${ }^{1}$ School of Physical and Health Education, Queen's University, Kingston, Ontario, Canada; the ${ }^{2}$ Department of Commumity Health and Epidemiobogy, Queen's University, Kingston, Ontario, Canada; the iCenters for Integrated Health Research, The Cooper lnstitute, Dallas, Texas; and the "Division of Endocrinology and Metabolism, Department of Medicine; Queen's University, Kingston, Ontario, Camada. Address correspondence and reprint requests to Robert Ross, FhD, Sthool of Physical and Health Education, Queen's Unversity, Kingstor, Onkario, Camada, K71. 3N6. E-muli: rossropost.queensu.ca. Received for publication 8 September 2004 and accepted in revised form 2 January 2005.

Abbreviations: CRF, carciorespiratory fitness.

A table elsewhere in this issue shows comentional and Systeme International (SD) units and conversion fectors for mary substances.

82005 by the American Dizbetes Association.

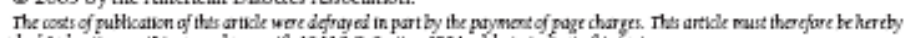

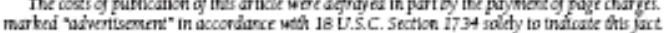

fold thickness and waist circumferenc compared with individuals with lowe CRF, independent of sex. Similarly Wong et al. (8) report that for a giver BMI, men with high CRF have lower tote abdominal, visceral, and abdominal sub cutaneous fat levels compared with mer with low CRF, a finding that remains tru for African-American men and wome (9). Although it is clear that high CRF i associated with lower levels of abdomina adiposity, the association between CRI and measures of metabolic health for given level of abdominal subcutaneou and/or visceral adiposity remains largel unknown.

Regular physical activity is an effec tive means of improving CRF and reduc ing waist circumference, visceral fat, an subcutaneous fat independent of a corre sponding change in BMI $(10,11)$. Fur thermore, regular physical activity is als associated with favorable changes it blood lipid profiles, blood pressure, an glucose metabolism; these improvement often occur with little or no improvemen in BMI (12). Recently, Nagano et al. (13 reported that high CRF is associated witl a lower risk of hyperinsulinemia and in creased HDL cholesterol levels after con trolling for visceral fat in Japanes individuals with glucose intolerance an type 2 diabetes. In that study, the author combined men and women in their ans yses and did not simultaneously contro for subcutaneous abdominal fat and/o waist circumference. Both abdomina subcutaneous fat (14) and waist circum ference (15) are reported to be strong, in dependent correlates of metabolic risk.

The primary purpose of this study was, therefore, to determine whethe higher levels of CRF are associated witl lower levels of selected metabolic risk fac tors for a given level of abdominal subcu taneous fat, visceral fat, and wais circumference. To examine this question we studied a sample of Caucasian met 
Exercise without weight loss is an effective strategy for obesity reduction in obese individuals with and without Type 2 diabetes

\author{
SoJung Lee, ${ }^{1}$ Jennifer L . Kuk, ${ }^{1}$ Lance E. Davidson, ${ }^{1}$ Robert Hudson, ${ }^{2}$ \\ Katherine Kilpatrick, ${ }^{1,3}$ Terry E. Graham, ${ }^{4}$ and Robert Ross ${ }^{1,2}$ \\ ${ }^{1}$ School of Physical and Health Edacation, and Divisions of ${ }^{2}$ Endocrinology and Metabolism, and \\ 'Geriatrics, Department of Medicine, Queen's Universily, Kingston, Ontario; and ${ }^{4}$ Department \\ of Human Biology and Nutritional Sciences, University of Guelph, Guelph, Ontario, Canada \\ Submitted 18 January 2005: acoepted in final form 19 April 2005
}

Lee, SoJung. Jennifer L. Kuk, Lance E. Davidson, Robert Hudson, Katherine Kilpatrick, Terry E. Graham, and Robert Ross. Exercise without weight loss is an effective strategy for obesity reduction in obese individuals with and without Type 2 diabetes. J Appl Physiol 99: 1220-1225, 2005. First published April 28, 2005. doi: 10.1152 japplphysiol.00053.2005. - It is unc bar whether chronic exercise without caloric restriction or weight loss is a useful strategy for cbesity reduction in obese men with and without Type 2 diabetes (T2D). We examined the effects of exercise without weight loss on total and regional adiposity and skeletal muscle mass and composition in lean men and in obese men with and without T2D. Twenty-four men participated in 13 wk of supervised aerobic exercise, five times per week for $60 \mathrm{~min}$ at a moderate intensity $(\sim 60 \%$ peak oxygen uptake) Total and regional body composition was measured by magnetic resonance imaging. Skeletal muscle composition was determined using computed tomography. Cardiorespiratory fitness was assessed using a graded maximal treadmill test. Body weight did not change within any group in response to exercise $(P>0.1)$. Significant reductions in total, abdominal subcutanecus, and visceral fat wer observed within each group $(P<0.01)$. The reduction in total and abdominal subcutaneous fat was not different $(P>0.1)$ between groups; however, the reduction in visceral fat was greater $(P<0.01)$ in the obese and T2D groups by comparison to the lean group. A significant $(P<0.01)$ increase in total skeletal muscle, high-density muscle area, and mean muscle attenuation was observed independent of grcup, and these changes were no different between groups $(P>$ $0.1)$. Accordingly, whole body fat-to-muscle ratio was increased $(P<$ 0.01 ) independent of groups In conclusion, regular exercise without weight loss is associated with a substantial reduction in total and visceral fat and in skeletal muscle lipid in both obesity and T2D.

total and abdominal fat; skeletal muscle composition

THE RREVAleNCE OF CBesity and Type 2 diabetes (T2D) is already high and continues to rise $(15,25)$. It is well known that physical inactivity is a primary cause of obesity $(16,22$. 24). Accordingly, adoption of a physically active lifestyle is now recognized as a cornerstone in the treatment and prevention of obesity $(7,27)$. Common to current obesity reduction guidelines is the assumption that the effectiveness of diet and/or exercise-based strategies depends in large measure on weight loss. Indeed, it is recommended that diet and exercise induce a negative energy balance that comesponds to a reduc-

Addres for reprint requeas and other carteppondenser R. Ross, School of Pddres for repnint requeas and other carteppondencer R. Rosk, School of Physical and Health Education, Queen's
K7L 3N6 (E-mail: roeg Epostqueensu.ca).

tion of $\sim 1.5 \mathrm{lb} .(0.6 \mathrm{~kg})$ per week (7). Furthermore, recent guidelines (19) highlight that weight loss should be a primary therapeutic strategy in all obese individuals who have T2D or are at risk for developing T2D. By definition, these guidelines (19) do not consider whether exercise alone is an effective strategy for obesity reduction even in the absence of weight loss. That weight loss is a desirable goal of obesity reduction is clear. However, the assumption that weight loss is the primary antecedent to lifestyle-induced benefit procludes, for example, the possibility that exercise could induce a reduction in waist circumference, a strong independent predictor of morbidity and mortality, without a corresponding reduction in body weight [e.g., body mass index (BMI)].

At present, little is known about the utility of regular exercise without corresponding weight loss as a strategy for the treatment of obesity. Our laboratory has recently reported that exercise without weight loss is associated with a significant reduction in abdominal fat in both obese men (29) and women (30), suggesting that regular exercise is associated with a clinically signific ant reduction in abdominal doesity without a corresponding change in BMI. Whether exercise without weight loss is associated with reduction in total and/or abdominal obesity in T2D is unknown.

Emerging evidence suggests that the accumulation of lipid within skeletal muscle increases with obesity $(9,11,13,17)$ and that muscle lipid content is associated with insulin resistance in lean $(26)$, obese $(11,12)$, and individuals with T2D (11). It has also been reported that both diet- $(10,13)$ or exercise-induced weight loss $(5)$ are associated with diminished skeletal muscle lipid content. However, whether exercise without weight loss is associated with a reduction in this ectopic lipid marker of metabolic risk is lacking and warrants investigation.

This study was undertaken to detemine the effects of exercise without weight loss on total and regional adiposity as well as skeletal muscle composition in lean men and in obese men with and without T2D. We hypothesized that exercise without weight loss would be associated with marked reduction in abdominal obesity and muscle lipid content in both obesity and T2D. Were this true, it would add important insight into the process by which the adoption of a physically

The costs of publication of this article were defrayed in part by the payment of page charges. The article must therefore be bereby marked "adver risement in accondance with 18 USC. Section 1734 solely to indicate this fact 


\section{Protocol for measurement of liver fat by computed tomography}

Lance E. Davidson, ${ }^{1}$ Jennifer L. Kuk, ${ }^{1}$ Timothy S. Church, ${ }^{3}$ and Robert Ross ${ }^{12}$

${ }^{1}$ School of Physical and Health Education and 'Division of Endocrinology and Metabolism, Department of Medicine, Qiveen's

University, Kingston, Ontario, Canada; and ' $\mathrm{C}$ Centers for Integrased Health Research, The Cooper Institude, Dallas, Texas

Submitted 12 Augus 2005: accepted in final form 6 November 2005

Davidson, Lance E., Jennifer L. Kuk, Timothy S. Church, and Robert Russ Protocol for measurement of liver fat by computed tomography. $J$ Appl Physiol 100: 864-868, 2006. First published tomography. $J$ Af November 17, 2005: doi: 10.1152/japplphysiol.00986.2005.-To develop a protocol for measurement of liver fat using computed tomography (CT), we conducted a preliminary study with 118 men and 76 women to determine a readily identifiable vertebral landmark at which the CT image displayed both liver and spleen. Analysis of five le CT ims simultaneously displayed the liver and spleen in $90 \%$ of the men and women. The $T_{12}-L_{1}$ protocol was cross-validated on a sample of 130 men and 113 women. In this sample. we also assessed the regional characteristics of liver and spleen tissue attenuation at the $T_{12}-L_{3}$ level by subdividing each image into quartiles from anterior to posterior, each of which were further divided into medial and lateral regions. A similar analys is was performed on images located $12 \mathrm{~mm}$ above and below $\mathrm{T}_{12}-\mathrm{L}_{1}$. The $\mathrm{T}_{12}-\mathrm{L}_{1}$ image displayed both liver and spleen in $92 \%$ ( 403 of 437 ) of the combined study sample. There was a significant $(P<0.005)$ stepwise increase in attenuation values [Hounsfield units (HU)] from the inferior to superior image. Although some significant $(P<0,05)$ differences were observed between the eight regions by comparison to the whole liver or spleen, the average magnitude of the difference was $<2.0 \mathrm{HU}$ for liver and $<3.5 \mathrm{HU}$ for spleen. Acquisition of a single CT image at the $\mathrm{T}_{12}-\mathrm{L}_{1}$ level is a practical and reliable method for routine measurement of liver fat in research and clinical settings.

liver attenuation; spleen; fatty liver, bepatic steatosis

EMERGING EVIDENCE sugGests that excess deposition of lipid in the liver may act as an "ectopic" site of fat distribution that independently predicts insulin resistance (18) and dyslipidemia (1, 11). The prevalence of excess liver fat or "fatty liver" approximates $34 \%$ in the general population (17) and $48 \%$ in obese cohorts (2). These observations underscore the importance of liver fat measurement in studies that seek to understand the health implications of complex obesity phenotypes. Although liver biopsy is often considered the gold standard for measurement of liver fat, it can be painful (5) and has notable mortality risk (6). Proton magnetic resonance spectroscopy is noninvasive and offers researchers a reliable tool for measuring liver fat in vivo. However, application of magnetic resonance spectroscopy requires exceptional technical expertise and is restricted to liver fat measures in specified regions of interest. Radiological imaging tochniques such as computed tomography $(C T)$ are obtained routinely in clinical settings, are easily interpreted without need for sophisticated image-analysis software, and provide estimates of liver fat that correlate well with needle biopsy, especially in subjects with increased liver fat (16). On the other hand, CT employs ionizing radiation, and

Address for reprint requeds and other correppondence: R. Ross, School of Physical and Healt Education, Queen's Univ , Kingston, Ortario, Canad Physical ond Heil tedion, Queen's thus protocols need to be developed that measure the tissues of interest and limit exposure.

The identification of liver fat by CT as a predictor of health risk was first described by Banerji et al. (1) and Goto et al. (7) in 1995. The CT method employed realizes that the lower the mean liver attenuation or CT number in Hounsfield units (HU), the lower the tissue density and hence the greater the fat content. Therefore, liver density (c.g., attenuation in HUs) is inversely related to liver fat and thus is a surrogate for it (15). However, although extremely low $\mathrm{HU}$ values have been measured in livers infiltrated with fat, an overlap exists between normal and abnomal liver HU values (13). Therefore, the absolute liver density determined by $\mathrm{CT}$ may not be sensitive for predicting abnormal liver fat content. Because a constant relationship exists between liver and spleen attenuation in individuals with normal livers, the ratio of mean liver to spleen attenuation values is used as an index of liver fat, as originally described by Pickarski et al. (14) in 1980. Obtaining a CT image that contains both liver and spleen presents a challenge: variations exist not only in the vertical positioning of the spleen relative to the liver but also in positioning of both organs within the abdominal cavity. A multi-image approach is not feasible because of excess exposure (8). This implicates a single CT image approuch: however, a single-image protocol at the level of the abdomen that routinely identifies the liver and spleen has yet to be fimly established. Furthermore, once obtained, it is important to determine whether the distribution of fat throughout the liver is unifom: an abservation with direct implications for those that determine liver fat by measuring only small portions or regions of interest of the liver on the CT image (e.g., biopsy, magnetic resonance spectroscopy, and CT)

The aim of this study was twofold: first, to determine an optimal location for simultaneous imaging of both liver and sploen, and second, to document liver and spleen attenuation characteristics and determine whether vanation exists, and if so, whether it is of a magnitude that should alter the protocol employed to measure liver fat.

\section{METHODS}

Sabject testing. Subjects were men and women who underwent medical examination at the Cooper Institute in Dallas. TX, between 1995 and 2001. Although race data for all subjects was unavailable, $\sim 98 \%$ of attendees at the Cooper Institute during this time were Caucasian. None of the subjects were smokers or had a history of diabetes mellitus, cardiovascular disease, stroke, or cancer. All gave informed consent before participation in the ex amination acoording to the ethical guidelines of The Cooper Institute Institutional Review Board. Medical examinations included an abdominal CT scan, conprising 40 contiguous images with 6 -mm thickness, from at least $T_{11}$

The costs of publication of this article were defrayed in part by the payment of page charges. The is in accondance with 18 USC. Section 1734 solety to indicate this fact 


\title{
Association of Cardiorespiratory Fitness, Body Mass Index, and Waist Circumference to Nonalcoholic Fatty Liver Disease
}

\author{
TIMOTHY S. CHURCH, ${ }^{*}$ JENNIFER L. KUK, ${ }^{\dagger}$ ROBERT ROSS, ${ }^{\dagger}$ ELISA L. PRIEST, ${ }^{*}$ EMILY BILTOFF, $\$$ \\ and STEVEN N. BLAIR* \\ *The Cocper Instutute, Dallas, Texas; †School of Physical and Health Education, Queen's Unmersity, Kngston, Ontarb, Canada; and 5Texas \\ Tech Unhersity School of Medicine, Lubbock, Texas
}

\section{See CME Quiz on page 2207.}

Background \& Aims: There is a need for more work examining the potential of physical activity and/or weight control as a preventive and/or therapeutic option in the treatment of fatty liver diseases. The purpose of this study was to examine the association between cardiorespiratory fitness, body mass index (BMI), and waist circumference with markers of nonalcoholic fatty liver disease (NAFLD). Methods: Participants consisted of 218 apparently healthy nonsmoking, nonalcoholic men aged $33-73$ years. Cardiorespiratory fitness was assessed by a maximal treadmill test. Liver and spleen density were measured using a computed tomography scan. We defined the presence of NAFLD as the following 3 conditions being met: (1) liver to spleen density of 1.0 or less, (2) serum alanine transaminase level greater than $30 \mathrm{U} / \mathrm{L}$, and (3) serum aspartate transaminase/alanine transaminase level less than 1.0. Results: Twenty-four (11\%) of the participants met the NAFLD definition. There was an inverse association be tween fitness categories, and a positive association for BMI categories (and waist circumference categories) with the prevalence of NAFLD ( $P$ for trend <.001 for all). Fitness and BMI were independent of each other in their associa tions with the prevalence of NAFLD. The addition of waist circumference to the regression model attenuated the association with prevalence of NAFLD for both fitness ( $P$ value changed from <.0001 to .06) and BMI ( $P$ value changed from <.001 to .22). Conclusions: Fitness (inversely) and BMI (directly) were associated with the prevalence of NAFLD. However, these associations were attenuated when abdominal obesity was included in the statistical model.

Tomalcoholic fatty liver disease (NAFLD) represents a 1 spectrum of conditions that span from asymptomatic steatosis to potentially deadly nonalcobolic steatohepatitis (NASH) ${ }^{1-6}$ Although simple steatosis is generally a benign condition, NASH can progress to cirthosis and liver failure, and the 5-year survival race for individuals diagnosed with $\mathrm{NASH}$ is estimated to be $67 \% .7 \mathrm{NASH}$ is distinguished from simple stearosis by the presence of bepatocyte injury, inflammation, and fibrosis. $5,6,8$ The prevalence of NAFLD and NASH in the general population is estimated to be $16 \%-30 \%$ and $2.1 \%-6.3 \%$, respectively. ${ }^{37,8} \mathrm{~A}$ liver biopsy examination is required for the diagnosis of NASH, which makes large-scale epidemiologic trials focused on $\mathrm{NASH}$ in asympromatic popularions problemaric, if not impossible, to conduct ethically. ${ }^{7}$

The prevalence of boch NAFLD and NASH have been shown to be associated strongly with excess body weight, in particular cencral adiposity. ${ }^{1,7,9-14}$ However, this associacion is complicared by body weight and central adiposity both being associated strongly (inversely) with regular physical activity, and physical axtivity is associated strongly (inversely) wich insulin resistance, which has been hypothesized to be a causal mechanism in NAFLD ${ }^{6-8}$ Previcus reports that have found overweight/obesity to be associaced with a higher prevalence of NAFLD eicher did not account for physical activity patterns or used self-report measures of physical activity, which leads to substantial misclassification on this exposure. ${ }^{1,12-14}$ Cardiorespiracory ficness is an objective laboracory measurement that reduces the misclassification bias that often results from self-reports of physical activity. ${ }^{15}$

The purpose of this study was to examine the associations among cardiorespiratory fitness, body mass index (BMI), and waist circumference with a marker of NAFLD in asymptomatic men. In combining computed tomography-measured liver fat with increases in liver enzyme levels we sought to create a surrogate measure of NAFLD that is closer to NASH in the NAFLD continuum than simple steatosis. With the increasing clinical presence of NAFLD and NASH, there is a need for more work examining the potential of physical activity and/or

\footnotetext{
Abtreviations Used in this poper: BMI, body mass index; L/S, I Wer/ spleen attenustion; MET, metabolic equivalent; NAFLD, nonalcoholic fatty Iver disease; NASH, nonalcoholic steatohepatitis.

o 2006 by the American Gastroenterological Association Institute $0016-5085 / 06 / \$ 32.00$ doi:10.1053/J.gastro.2006.03.019
} 
Appendix N: List of Publications and Abstracts 


\section{MANUSCRIPTS}

1. Kuk JL, Janiszewski PM and Ross R: The Independent Associations of Waist Circumference, Body Mass Index and Hip Circumference with Total and Regional Measures of Body Composition in Men and Women. (Am J Clin Nutr - In Press).

2. McMillan KP, Kuk JL, Church TS, Blair SN and Ross R: The Independent Associations between Liver Fat, Visceral Adipose Tissue and Metabolic Risk Factors in Men. (APNM - In Press).

3. Church TS, Kuk JL, Ross R, Priest EL, Biltoff E, Blair SN: The Relative Association of Cardiorespiratory Fitness, Body Mass Index and Waist Circumference to Nonalcoholic Fatty Liver Disease in Men. (Gastroenterology 130:2023-2030, 2006).

4. Kuk JL, Katzmarzyk PT, Nichaman MZ, Church TS, Blair SN, and Ross R.: Visceral Fat is an Independent Predictor of All-Cause Mortality in Men. (Obes Res 14: 336-341, 2006).

5. Kuk JL, Church TS, Blair SN, and Ross R: Does the Measurement Site for Visceral and Abdominal Subcutaneous Adipose Tissue Alter the Associations with the Metabolic Syndrome? (Diabetes Care 29: 679-684, 2006).

6. Davidson LE, Kuk JL, Church TS, Blair SN, and Ross R: Protocol for Measurement of Liver Fat by Computed Tomography. (J Appl Physiol 100:864-8, 2006).

7. Lee S, Kuk JL, Davidson LE, Hudson R, Kilpatrick K, Graham TE, Ross R.: Exercise without weight loss is an effective strategy for obesity reduction in obese individuals with and without type 2 diabetes. (J Appl Physiol. 99:1220-1225, 2005).

8. Kuk JL, Lee SJ, Heymsfield SB, Ross R.: Waist Circumference and Abdominal Adipose Tissue Distribution: Influence of Age and Gender. (Am J Clin Nutr 81(6):1330-1334, 2005).

9. Lee SJ, Kuk JL, Katzmarzyk PT, Blair SN, Church TS, Ross R.: Cardiorespiratory Fitness Attenuates Metabolic Risk Independent of Abdominal Subcutaneous and Visceral Fat in Men. (Diabetes Care 28(4):895-901, 2005).

10. Kuk JL, Nichaman MZ, Church TS, Blair SN, and Ross R.: Liver fat is not a marker of metabolic risk in lean premenopausal women. (Metabolism 53:1066-71, 2004).

11. Ross R, Janssen I, Dawson J, Kungl AM, Kuk JL, Wong S, Nguyen-Duy TB, Lee SJ, Kilpatrick K, Hudson R: Exercise with or without weight loss is associated with reduction in abdominal and visceral obesity in women. A randomized controlled trial. (Obes Res 12:789-98, 2004).

\section{BOOK CHAPTERS}

1. Kuk JL and Ross R.: Body Composition in Obesity, Editors: Kushner R and Bessesen DH. Contemporary Endocrinology: Treatment of the Obese Patient. Humana Press (In Press).

\section{REVIEW CHAPTERS}

1. Kuk JL, Janiszewski PM and Ross R.: Exercise, Visceral Adipose Tissue and Metabolic Risk. Current Cardiovascular Risk Reports. (In Press). 


\section{PUBLISHED ABSTRACTS AND CONFERENCE PROCEEDINGS}

1. Kuk JL, Church TS, Blair SN, and Ross R: Associations between Exercise-induced Changes in the Quantity and Quality of Abdominal and Thigh Muscle in Postmenopausal Women. (Appl Physiol Nutrition Metabolism 31:S50, 2006).

2. Janiszewski PM, Kuk JL and Ross R: The health-protective effect of a large hip circumference for a given waist circumference: Is visceral fat a mediator? (App Physiol Nutrition Metabolism 31:S39, 2006).

3. Kuk JL, Church TS, Blair SN, and Ross R: Influence of Exercise Dose and Cardiorespiratory Fitness on Changes in Abdominal Adiposity in Postmenopausal Women - The DREW Study. (Obesity 14:A169, 2006).

4. Davidson LE, Kuk JL, Hudson R, Kilpatrick K, and Ross R: Effect of Dietary Fat Intake on the Relationship between Liver Fat and Insulin Sensitivity in Sedentary, Abdominally Obese Older Men. (Obesity 14:A147, 2006).

5. Janiszewski PM, Kuk JL, Ross R: Is reduction in lower body fat associated with exacerbation in cardio-metabolic risk? (Obesity 14:A44-45, 2006).

6. McMillan KP, Kuk JL, Church TS, Blair SN, and Ross R: Independent Associations between Cardiorespiratory Fitness, Liver Fat, Visceral Fat and Metabolic Risk in Men. (Med Sci Sports Exerc 38(5):S203, 2006).

7. Janiszewski PM, Kuk JL, Oeffinger KC, Church TS, Ross R: Regional Fat Deposition, Cardiorespiratory Fitness and Insulin Resistance in Adult Survivors of Childhood Leukemia. (Med Sci Sports Exerc 38(5):S203-204, 2006).

8. Kuk JL, Katzmarzyk PT, Nichaman MZ, Church TS, Blair SN, and Ross R: Visceral Fat is an Independent Predictor of All-Cause Mortality in Men. (Can J Appl Physiol 30:S45, 2005).

9. Davidson LE, Kuk JL, Church TS, Blair SN, and Ross R: Novel Assessment Of Hepatic Steatosis By Computed Tomography. (Can J Appl Physiol 30:S20, 2005).

10. McMillan KP, Kuk JL, Church TS, Blair SN, and Ross R: Independent Associations between Visceral Fat, Liver Fat and Metabolic Risk Factors in Men. (Can J Appl Physiol 30:S55-56, 2005).

11. Kuk JL, Church TS, Blair SN, and Ross R: Does the Measurement Site for Visceral and Abdominal Subcutaneous Adipose Tissue Alter the Associations with the Metabolic Syndrome? (Obes Res, 13:A126, 2005).

12. Davidson LE, Kuk JL, Kilpatrick K, Hudson R, Ross R: Independent Relationships Among Abdominal Adipose Depots, Liver Fat, Cardiorespiratory Fitness, and Insulin Resistance in Abdominally Obese Older Men and Women. (Obes Res 13:A64, 2005).

13. Kuk JL, Church TS, Blair SN, and Ross R: Pancreas Fat and Liver Fat are Independent Predictors of the Metabolic Syndrome and Type II Diabetes in Men. (Can J Diabetes, S56, 2005).

14. Ross R, Kuk JL, Church TS, Blair SN: Measurement Protocol for Measurement of Visceral and Abdominal Subcutaneous Adipose Tissue: Influence on Associations with the Metabolic Syndrome (IJBCR 3(3):88, 2005).

15. Church TS, Jordan A, Priest E, LaMonte MJ, Kuk JL, Ross R, Blair SN: The Prevalence of Nonalcoholic Steatohepatitis Across Levels of Cardiorespiratory Fitness In Men. (Med Sci Sports Exerc 37(5):S207-8, 2005).

16. Walker KL, Saunders NR, Jensen D, Kuk JL, Wong SL, Pyke KE, Dwyer EM, Tschakovsky ME: Vasoregulatory Responses to Sudden Changes in Forearm Perfusion Pressure During Moderate Forearm Exercise. (Med Sci Sports Exerc 37(5):S220, 2005). 
17. Kuk JL, Lee SJ, Heymsfield SB, Ross R.: Relationship between age, gender, waist circumference and abdominal adipose tissue distribution. (Obes Res, 12:A210, 2004).

18. Lee SJ, Kuk JL, Katzmarzyk PT, Blair SN, Church TS, Ross R.: Cardiorespiratory Fitness Attenuates Metabolic Risk Independent of Abdominal Subcutaneous and Visceral Fat in Men. (Obes Res, 12:A153, 2004).

19. Kilpatrick K, Kuk JL, Hudson R, Ross R.: Skeletal Muscle Mass is not Related to Glucose Tolerance or Insulin Sensitivity in Overweight and Obese Men and Women. (Obes Res, 12:A138, 2004).

20. Davidson LE, Kuk JL, Ross R.: Liver Volume as a Marker of Hepatic Fat in Men. (Obes Res, 12:A137, 2004).

21. Kuk JL, Katzmarzyk P, Hudson R, Kilpatrick K, Ross R.: Metabolically abnormal and metabolically normal obese men and women: differences are not explained by total or abdominal adiposity. (Can J Appl Physiol 28: S74, 2003).

22. Kuk JL, Nichaman MZ, Church TS, Blair SN, and Ross R.: Independent Contribution of Visceral Adiposity to the Lipid Profile of Premenopausal Women: Evidence Against the "Portal Theory". (Can J Appl Physiol 27: S29, 2002).

23. Kuk JL, Blair SN, Church TS, Ross R.: Independent contribution of Abdominal Obesity, Liver Fat and Cardiorespiratory Fitness to the Lipid Profile of Premenopausal Women. (Canadian Federation of Biological Sciences, 2002). 